\title{
Estimations of the Conditional Tail Average Treatment Effect*
}

\author{
Le-Yu Chen ${ }^{\dagger}$ \\ Academia Sinica
}

\author{
Yu-Min Yen ${ }^{\ddagger}$ \\ National Chengchi University
}

September 23, 2021

\begin{abstract}
We study estimation of the conditional tail average treatment effect (CTATE), defined as a difference between conditional tail expectations of potential outcomes. The CTATE can capture heterogeneity and deliver aggregated local information of treatment effects over different quantile levels, and is closely related to the notion of second order stochastic dominance and the Lorenz curve. These properties render it a valuable tool for policy evaluations. We consider a semiparametric treatment effect framework under endogeneity for the CTATE estimation using a newly introduced class of consistent loss functions jointly for the conditioanl tail expectation and quantile. We establish asymptotic theory of our proposed CTATE estimator and provide an efficient algorithm for its implementation. We then apply the method to the evaluation of effects from participating in programs of the Job Training Partnership Act in the US.
\end{abstract}

JEL classification: C13, C14, C21

Keywords: Causal inference, Conditional tail expectation, Endogeneity, Semiparametric estimation, Treatment effects.

${ }^{*}$ We thank seminar participants in 2019 macroeconometric modelling workshop (Academia Sinica), 2020 Annual Meeting of Taiwan Econometric Society, National Chengchi University and National Taiwan University helpful comments.

${ }^{\dagger}$ Institute of Economics, Academia Sinica, 128, Section 2, Academia Road, Nankang, Taipei 115, Taiwan. E-mail: lychen@econ.sinica.edu.tw

${ }^{\ddagger}$ Department of International Business, National Chengchi University, 64, Section 2, Zhi-nan Road, Wenshan, Taipei 116, Taiwan. E-mail: yyu_min@nccu.edu.tw 


\section{Introduction}

The treatment effect of a policy change is often heterogeneous across individuals. Accounting for such heterogeneity is crucial for evaluating effects of and understanding mechanisms underlying the policy (Heckman et al., 1997). To capture heterogeneity of treatment effects, the quantile treatment effect (QTE, Abadie et al. (2002), Chernozhukov and Hansen (2005)) is a frequently used measure, which is computed as a difference between a given quantile of distribution of the potential outcome subject to the policy being changed and that of the potential outcome under the policy had it not been changed. In this paper, we consider an alternative measure for gauging heterogeneous treatment effects: the conditional tail average treatment effect (CTATE). The CTATE is defined as a difference between conditional tail expectations of two potential outcomes at a given quantile level. The CTATE amounts to a (rescaled) integral of the QTE over a specified range of quantiles and is thus useful for delivering aggregated local information of treatment effects.

The identification of the CTATE relies on using a class of consistent loss functions for jointly estimating the quantile and conditional tail expectation of a random variable proposed by Fissler and Ziegel (2016) (henceforth the FZ loss). With the FZ loss, we develop a semiparametric estimation procedure to jointly estimate the CTATE and QTE under the two-sided noncompliance framework (see e.g., Imbens and Rubin, 2015, Chapter 24), which is commonly employed in addressing endogeneity with instrumental variables and in developing various estimators for the local treatment effects in the causal inference literature (Imbens and Angrist, 1994; Angrist et al. 1996; Abadie et al., 2002; Abadie, 2003, Frölich and Melly, 2013; Belloni et al., 2017; Frölich and Huber, 2017; Chen et al., 2020; Fricke et al., 2020; Kianian et al., 2021; Wei et al., 2021). Under the framework, if some regularity conditions hold (Abadie et al., 2002; Abadie, 2003), endogeneity can be eliminated within samples of a subgroup (compliers), and with a weighted sampling scheme, econometric methods can be applied to locally estimate the interested causal parameters. We then establish asymptotic theory of the resulting CTATE estimator and provide an efficient and stable algorithm for its implementation.

We now further remarks on usefulness of the CTATE in empirical policy evaluation research. Conditional tail expectation is related to the notion of second order stochastic dominance. Stochastic dominance is a uniform order relation between distributions of stochastic outcomes and is often used in ranking individuals' preferences under uncertainty. Conventionally, formulations of the first and second order stochastic dominances (FOSD and SOSD) are based on properties of cumulative distributions of competing random outcomes. Such formulations can also be equivalently established via their quantiles and conditional tail expectations (Levy, 2016). For policy evaluations, if the profile of QTE is nonnegative over all quantile levels, policy being changed first order stochastically dominates policy that had not been changed $1 \frac{1}{1}$ Likewise, if the profile of CTATE is nonnegative over all quantile levels, we can then attest that policy being changed second order stochastically dominates policy that had not been changed. Therefore we can use

\footnotetext{
${ }^{1}$ We say policy A first order stochastically dominates policy B if individuals with an increasing utility function can achieve a higher expected utility level under A than B; policy A second order stochastically dominates policy $\mathrm{B}$ if individuals with an increasing and risk averse utility function can achieve a higher expected utility level under A than B.
} 
the CTATE profile to rank counterfactual outcome distributions in the SOSD sense when ranking by the FOSD criterion is empirically inconclusive.

The CTATE in two quantile levels can directly be used to calculate an average of quantile treatment effects between the two quantile levels, which we term the interquantile average treatment effect (IQATE). The IQATE summarizes local information of heterogeneous treatment effects and is useful on a policy evaluation, especially when the treatment effects fluctuate a lot in different quantile levels and tools based on the treatment effects in individual quantile levels render result of the evaluation inconsistent. Moreover, related to distributional comparison of economic outcomes, profile of the conditional tail expectations is also directly connected to the Lorenz curve for comparing income inequalities. The proposed procedure for estimating the CTATE may hence be used to quantify the Lorenz effect, which captures the shift of the Lorenz curve due to change of policy regimes (Chernozhukov et al., 2013).

In this paper, we estimate conditional tail expectations of potential outcomes through minimization of the FZ loss (Fissler and Ziegel, 2016). To the best of our knowledge, the FZ loss is so far the only known class of consistent loss functions for estimating conditional tail expectation of a random variable. Using consistent loss for estimating parameters of statistical functionals results in an M-estimation problem, which facilitates computation and statistical analysis of parameter estimators through the lens of empirical risk minimization. The conditional tail expectation, as a parameter of interest, has already received considerable attention in finance and risk management. In particular, conditional tail expectation of an asset's return, known as the expected shortfall, is now a benchmark risk assessment measure in the industry. In the previous literature, estimation of expected shortfalls was often based on estimated truncated means of assets' returns (see e.g., Linton and Xiao, 2013; Hill, 2015). Recently, there has been a growing interest in structural models for forecasting the expected shortfall using the FZ loss (Patton et al., 2019; Taylor, 2019; Meng and Taylor, 2020; Chou et al., 2020). These papers address the problem of finding the best model for the prediction of expected shortfall. By contrast, our paper, which also builds upon the FZ loss minimization approach, focuses on the CTATE estimation in a causal inference framework and thus have a fundamentally different objective from these previous works.

The rest of this paper proceeds as follows. In Section 2, we introduce the notion of CTATE, set forth the causal framework, and present our estimation approach as well as the implementation algorithm. In Section 3, we establish asymptotic properties of our CTATE estimator. In Section 4, we conduct simulation experiments to examine finite-sample performance of the proposed method. In Section 5, we illustrate usefulness of our approach in an empirical application using the job training partnership act (JTPA) data. We then conclude the paper in Section 6. Proofs of all technical results are relegated to the Appendix.

\section{Methodology}

\subsection{The conditional tail average treatment effect}

We follow the Rubin causal model with potential outcomes (see e.g., Imbens and Rubin, 2015 , Chapter 1). Let $D \in \mathcal{D}$ denote the treatment status, where $\mathcal{D}$ is a finite collection of treatment 
statuses. We use $Y_{d}$ to denote the potential outcome under the $d$ th treatment, where $d \in \mathcal{D}$, and $Y:=\sum_{d \in \mathcal{D}} 1\{D=d\} Y_{d}$ to denote the observed outcome. Our primary interest is the conditional tail average treatment effect (CTATE), which is the difference between the conditional tail expectations of $Y_{d}$ at a quantile level $\tau$, given the exogenous variables $X$.

To formally define the CTATE, let $Q_{Y_{d} \mid X}(\tau)$ denote the $\tau$-quantile of the distribution of the potential outcome $Y_{d}$ conditional on $X$. Let $C T E_{Y_{d} \mid X}(\tau):=E\left[Y_{d} \mid X, Y_{d} \leq Q_{Y_{d} \mid X}(\tau)\right]$ denote the conditional tail expectation of $Y_{d}$ at the $\tau$-quantile level, given $X$. Note that, for any continuous random variable $Y_{d}$

$$
C T E_{Y_{d} \mid X}(\tau)=\frac{1}{\tau} \int_{0}^{\tau} Q_{Y_{d} \mid X}(u) d u
$$

We define the CTATE under treatments $d$ and $d^{\prime},\left(d, d^{\prime}\right) \in \mathcal{D}$ at the $\tau$ th quantile level as

$$
\operatorname{CTATE}\left(\tau ; d, d^{\prime}\right):=C T E_{Y_{d} \mid X}(\tau)-C T E_{Y_{d^{\prime}} \mid X}(\tau)
$$

In the literature, the quantile treatment effect (QTE) under treatments $d$ and $d^{\prime}$ conditional on $X$ at the $\tau$ th quantile is defined as

$$
Q T E\left(\tau ; d, d^{\prime}\right):=Q_{Y_{d} \mid X}(\tau)-Q_{Y_{d^{\prime}} \mid X}(\tau)
$$

Using (1), (2) and (3), we note that CTATE is related to QTE through the equation:

$$
\operatorname{CTATE}\left(\tau ; d, d^{\prime}\right)=\frac{1}{\tau} \int_{0}^{\tau} Q T E\left(u ; d, d^{\prime}\right) d u .
$$

Therefore, if the function $Q_{Y_{d} \mid X}(\tau)$ is linear in quantile-specific parameters (e.g., Chernozhukov and Hansen (2006), Chernozhukov et al. (2007), Chernozhukov and Hansen (2008) and Chernozhukov et al. (2009)), then both $C T E_{Y_{d} \mid X}(\tau)$ and $C T A T E\left(\tau ; d, d^{\prime}\right)$ are also linear in quantilespecific parameters. Accordingly, for such a linear specification setting, if the treatment status $D$ is also exogenous, we can consistently estimate the structural parameters of the function $Q_{Y_{d} \mid X}(\tau)$ by minimizing the check loss for the standard quantile regression of Koenker and Bassett (1978) and estimate those of $C T E_{Y_{d} \mid X}(\tau)$ through minimizing the FZ loss. This then allows for consistent estimation of the corresponding QTE and CTATE. However, in the presence of endogeneity, such an estimation procedure may be invalid and bias the results.

\subsection{An estimation model for CTATE under two-sided noncompliance}

We consider treatment effect estimation in a two-sided noncompliance framework where the treatment status is potentially endogenous. In what follows, we focus on the case with binary treatment status $\mathcal{D}=\{0,1\}$ where an individual is deemed treated if and only if her treatment status takes value 1 . Assume there is also a binary instrumental variable $Z \in\{0,1\}$, which acts as an indicator for a random assignment of eligibility for receiving a treatment.

Given noncompliance, there are four types $(T)$ of individuals in the population: compliers $(T=c)$, always-takers $(T=a)$, never-takers $(T=n e)$ and defiers $(T=d e)$. Always-takers will and never-takers will not be treated regardless of their eligibility to receive the treatment. 
Compliers will take the treatment only if they are eligible and defiers will do so only if they are not eligible. Let $D_{z}, z \in\{0,1\}$, denote potential treatment statuses under eligibility assignment $z$. We can then summarize the relations between types $T$ and the potential treatment statuses $D_{1}$ and $D_{0}$ as: (1) $D_{1}=D_{0}=1$ if and only if $T=a$; (2) $D_{1}=D_{0}=0$ if and only if $T=n e$; (3) $D_{1}=1$ and $D_{0}=0$ if and only if $T=c$; (4) $D_{1}=0$ and $D_{0}=1$ if and only if $T=d e$.

Following Abadie et al. (2002), we make the following assumptions for identifying treatment effects for the compliers under the two-sided noncompliance framework:

Assumption 1 (Abadie et al. (2002)) For almost every realization of $X$ :

1. $\left(Y_{1}, Y_{0}, D_{1}, D_{0}\right) \perp Z \mid X$.

2. $0<P(Z=1 \mid X)<1$.

3. $P\left(D_{1}=1 \mid X\right) \neq P\left(D_{0}=1 \mid X\right)$.

4. $P\left(D_{1} \geq D_{0} \mid X\right)=1$.

Assumptions akin to those stated above are common in the literature of the Rubin causal model with instrumental variables (see e.g., Imbens and Angrist, 1994; Angrist et al., 1996; Abadie, 2003 , Imbens and Rubin, 2015, Chapter 24). Assumption 1.1 is analogous to an exclusion restriction requiring that, given $X$, the instrument $Z$ should not directly affect both the potential outcomes and potential treatment statuses. Thus the effect of $Z$ on the outcome $Y$ can only be through that on the observed treatment status $D$. This assumption holds when $Z$ is randomly assigned conditional on the covariates $X$. Assumptions 1.2 and 1.3 are mild and ensures that the relevance condition: $Z$ and $D$ are correlated conditional on $X$, holds. Assumption 1.3 also guarantees that $P(T=c \mid X)>0$ (Abadie, 2003) so that there is a nonzero proportion of compliers in the population. Assumption 1.4 is a monotonicity condition, which implies the potential treatment status $D_{z}$ is weakly increasing with $z$, thereby ruling out the existence of defiers. This condition is plausible in various applications. See e.g., Abadie et al. (2002) and Abadie (2003) for further discussions on these assumptions.

Under Assumption 1, Abadie et al. (2002) show that unconfounded treatment selection holds within the group of compliers:

$$
\left(Y_{1}, Y_{0}\right) \perp D \mid X, T=c .
$$

Therefore, for the group of compliers, the distribution of potential outcome $Y_{d}$ given $X$ amounts to that of the observed outcome $Y$ given $X$ and $D=d$, which enables us to derive causal parameters of interest for the compilers. ${ }^{2}$ In the present paper, we focus on the estimation of CTATE for the complier group. If the complier quantile regression model is specificed as linear-in-parameters

$$
Q_{Y \mid D, X, T=c}(\tau)=\alpha_{1, \tau} D+X^{\top} \boldsymbol{\beta}_{1, \tau},
$$

it then follows from (1) that the complier conditional tail expectation given $D$ and $X$ is also linear

\footnotetext{
${ }^{2}$ One may be concerned about whether Assumption 1 is more restricted than those for identifying treatment effects for the whole population, such as the assumptions for the instrumental variable quantile regression model of Chernozhukov and Hansen (2005). Wüthrich (2020) show that the two sets of assumptions are not nested within each other.
} 
in parameters such that

$$
C T E_{Y \mid D, X, T=c}(\tau)=\alpha_{2, \tau} D+X^{\top} \boldsymbol{\beta}_{2, \tau}
$$

where

$$
\alpha_{2, \tau}=\frac{1}{\tau} \int_{0}^{\tau} \alpha_{1, u} d u, \boldsymbol{\beta}_{2, \tau}=\frac{1}{\tau} \int_{0}^{\tau} \boldsymbol{\beta}_{1, u} d u .
$$

In model (7), we are mainly concerned with the scalar parameter $\alpha_{2, \tau}$, which delivers the $\tau$ th quantile level CTATE for the group of compliers. However, this model is not directly applicable in estimation as the group of compliers are generally not observed in the data. To address this issue, we follow the weighting scheme of Abadie et al. (2002) and Abadie (2003), who show that under Assumptions 1, given any function $h(Y, D, X)$ with finite mean, the following result holds:

$$
E[h(Y, D, X) \mid T=c]=\frac{1}{P(T=c)} E[K(D, Z, X) h(Y, D, X)],
$$

where

$$
\begin{aligned}
K(D, Z, X) & =1-\frac{D(1-Z)}{1-\pi(X)}-\frac{(1-D) Z}{\pi(X)} \\
\pi(X) & =P(Z=1 \mid X) .
\end{aligned}
$$

Exploiting this result, we can therefore estimate the parameters in (7) by minimizing a sample analogue of the numerator term of (8) with the function $h$ being a loss function for the estimation of conditional tail expectation. For this purpose, we propose using the FZ loss (Fissler and Ziegel, 2016), which then leads to a weighted empirical FZ loss minimization based estimation problem. Before we formally set forth the FZ loss in the next section, we note that the weight $K(D, Z, X)$ may not be non-negative for all realizations of $(D, Z, X)$, thus rendering the estimation problem computationally difficult. Following Abadie et al. (2002), we replace $K(D, Z, X)$ with

$$
\begin{aligned}
\bar{K}(Y, D, X) & =E[K(D, Z, X) \mid Y, D, X] \\
& =1-\frac{D(1-E[Z \mid Y, D, X])}{1-\pi(X)}-\frac{(1-D) E[Z \mid Y, D, X]}{\pi(X)}
\end{aligned}
$$

It is straightforward to see that

$$
E[\bar{K}(Y, D, X) h(Y, D, X)]=E[K(D, Z, X) h(Y, D, X)]
$$

In addition, under Assumption 1, we have that $\bar{K}(Y, D, X)=P(T=c \mid Y, D, X)$ so that the projected weight $\bar{K}(Y, D, X)$ is non-negative almost surely.

For practical implementation, we plug in consistent estimators of the unknown components $\pi(X)$ and $E(Z \mid Y, D, X)$ of 11 to estimate $\bar{K}(Y, D, X)$. Let $\hat{\bar{K}}_{i}:=\hat{\bar{K}}\left(Y_{i}, D_{i}, X_{i}\right)$ denote such a plug-in estimator evaluated at the $i$ th observation. We will show in Section 3 that $\hat{\bar{K}}$ is consistent for $\bar{K}$ under certain regularity conditions. However, the estimated weight $\hat{\bar{K}}_{i}$ might not yet be guaranteed to lie within the interval $(0,1)$ for every observation in finite samples. If $\hat{\bar{K}}_{i}>1$ or $\hat{\bar{K}}_{i}<0$, we will then further truncate it by resetting its value to be 1 or 0 accordingly. 


\subsection{The FZ loss function}

The FZ loss (Fissler and Ziegel, 2016) is a class of consistent loss functions for eliciting both the qunatile and the conditional tail expectation of a random variable, meaning that these two statistical functionals can be jointly identified by minimizing expectation of the FZ loss. Interestingly, unlike the quantile, which can be elicited with some consistent loss functions (say, the check loss), there does not yet exist a consistent loss function solely for eliciting the conditional tail expectation (Fissler and Ziegel, 2016).

Let $\mathcal{F}$ be a class of distribution functions on the real numbers $\mathbb{R}$ with finite first moments and unique $\tau$-quantiles. For a random variable $Y$ following a distribution $F \in \mathcal{F}$, let $Q_{Y}(\tau)$ denote the $\tau$-quantile and $C T E_{Y}(\tau)$ denote the corresponding conditional tail expectation. Fissler and Ziegel (2016) show that

$$
\left(Q_{Y}(\tau), C T E_{Y}(\tau)\right)=\arg \min _{(q, e)} E\left[F Z_{\tau}(q, e, Y)\right]
$$

where

$$
\begin{aligned}
F Z_{\tau}(q, e, y)= & 1\{y \leq q\}\left[G_{1}(q)-G_{1}(y)\right]-\tau G_{1}(q) \\
& +G_{2}^{\prime}(e)\left[e+L Q_{\tau}(q, y)\right]-G_{2}(e)+\eta(y)
\end{aligned}
$$

is the FZ loss. Here $L Q_{\tau}(q, y):=\tau^{-1} \max (q-y, 0)-q, \tau \in(0,1)$ is the quantile level, $(q, e, y) \in$ $\mathbb{R}^{3}$ and $e \leq q . G_{1}(),. G_{2}($.$) and \eta($.$) are functions of real numbers and G_{2}^{\prime}($.$) is the subgradient$ of $G_{2}($.$) . It is required that 1_{[-\infty, q)} G_{1}($.$) be F$ integrable for all $q \in \mathbb{R}$ and all $F \in \mathcal{F}$, and $G_{2}^{\prime}($. and $\eta$ (.) be $F$ integrable for all $F \in \mathcal{F}$. In addition, $G_{1}$ (.) should be increasing and $G_{2}($.$) should$ be increasing and convex. If $G_{2}($.$) is both strictly increasing and strictly convex, the minimizer in$ (13) is unique and the loss function $F Z_{\tau}(q, e, y)$ is said to be strictly consistent for the $\tau$-quantile and the corresponding conditional tail expectation of all $F \in \mathcal{F}($ Fissler and Ziegel, 2016).

Let $\boldsymbol{\theta}_{1, \tau}=\left(\alpha_{1, \tau}, \boldsymbol{\beta}_{1, \tau}^{\top}\right)^{\top}$ and $\boldsymbol{\theta}_{2, \tau}=\left(\alpha_{2, \tau}, \boldsymbol{\beta}_{2, \tau}^{\top}\right)^{\top}$ be the vectors that collect the true parameters of models (6) and (7). Using the weighted loss minimization approach of Section 2.2, we can estimate $\boldsymbol{\theta}_{1, \tau}$ and $\boldsymbol{\theta}_{2, \tau}$ by minimizing the sample analog of the left-hand side of $(12)$, with the function $h$ being replaced by the FZ loss 14 . Specifically, let $\hat{\boldsymbol{\theta}}_{1, \tau}$ and $\hat{\boldsymbol{\theta}}_{2, \tau}$ denote the estimators of $\boldsymbol{\theta}_{1, \tau}$ and $\boldsymbol{\theta}_{2, \tau}$. Assume the data consist of a sample of $n$ observations $\left(Y_{i}, D_{i}, X_{i}, Z_{i}\right), i=1, \ldots, n$. The estimators $\hat{\boldsymbol{\theta}}_{1, \tau}$ and $\hat{\boldsymbol{\theta}}_{2, \tau}$ can then be computed as the solution to the problem

$$
\min _{\left(\boldsymbol{\theta}_{1}, \boldsymbol{\theta}_{2}\right)} \frac{1}{n} \sum_{i=1}^{n} \tilde{K}_{i} F Z_{\tau}\left(q_{i}\left(\boldsymbol{\theta}_{1}\right), e_{i}\left(\boldsymbol{\theta}_{2}\right), Y_{i}\right)
$$

where $q_{i}\left(\boldsymbol{\theta}_{1}\right)=q\left(D_{i}, X_{i}, \boldsymbol{\theta}_{1}\right):=\alpha_{1} D_{i}+X_{i}^{\top} \boldsymbol{\beta}_{1}$ and $e_{i}\left(\boldsymbol{\theta}_{2}\right)=e\left(D_{i}, X_{i}, \boldsymbol{\theta}_{1}\right):=\alpha_{2} D_{i}+X_{i}^{\top} \boldsymbol{\beta}_{2}$ are regression functions of (6) and (7) evaluated at parameter values $\boldsymbol{\theta}_{1}=\left(\alpha_{1}, \boldsymbol{\beta}_{1}^{\top}\right)^{\top}$ and $\boldsymbol{\theta}_{2}=$ $\left(\alpha_{2}, \boldsymbol{\beta}_{2}^{\top}\right)^{\top}$, and $\tilde{K}_{i}=\min \left\{\max \left\{\hat{\bar{K}}_{i}, 0\right\}, 1\right\}$ is an estimate of $\bar{K}\left(Y_{i}, D_{i}, X_{i}\right)$ truncated to take value in the interval $[0,1]$. 


\subsection{A specification of the FZ loss and an implementation algorithm}

To practically implement the estimation, we need to specify functional forms of $G_{1}(),. G_{2}($.$) and$ $\eta($.$) . In the present paper, we set G_{1}(t)=0, G_{2}(t)=\eta(t)=\ln (1+\exp (t))$ (the softplus function) for $t \in \mathbb{R}$, which enables us to solve the problem (15) through a simple iterative computation algorithm. Under these settings, the loss function $F Z_{\tau}(q, e, y)$ becomes

$$
F Z_{\tau}^{s p}(q, e, y)=\frac{\exp (e)}{1+\exp (e)}\left[e+L Q_{\tau}(q, y)\right]-\ln [1+\exp (e)]+\ln [1+\exp (y)] .
$$

The loss function $(16)$ is defined for all $(q, e, y) \in \mathbb{R}^{3}$. Since $G_{2}($.$) is the softplus function, it is$ straightforward to verify that $F Z_{\tau}^{s p}(q, e, y)$ is a strictly consistent loss function. Moreover, using the fact that $F Z_{\tau}(q, e, y) \geq 0$ whenever $\eta(y)=\tau G_{1}(y)+G_{2}(y)$ (Dimitriadis and Bayer, 2019), it follows that $F Z_{\tau}^{s p}(q, e, y)$ is a non-negative function as well.

With the FZ loss specification (16), we solve the following minimization problem to obtain estimates of $\boldsymbol{\theta}_{1, \tau}$ and $\boldsymbol{\theta}_{2, \tau}$ :

$$
\min _{\left(\boldsymbol{\theta}_{1}, \boldsymbol{\theta}_{2}\right)} \frac{1}{n} \sum_{i=1}^{n} \tilde{K}_{i} F Z_{\tau}^{s p}\left(q_{i}\left(\boldsymbol{\theta}_{1}\right), e_{i}\left(\boldsymbol{\theta}_{2}\right), Y_{i}\right)
$$

However, joint minimization over $\left(\boldsymbol{\theta}_{1}, \boldsymbol{\theta}_{2}\right)$ in (17) could be computationally nontrivial, since the function $L Q_{\tau}(q, y)$ involves the term $\max (q-y, 0)$, which has a kink and is thus not everywhere differentiable. Patton et al. (2019) adopted a smoothed approximation to the objective function in their FZ loss minimization problem. They solved the smoothed problem to obtain an approximate solution, which is then used as an initial guess in a non-gradient based numerical procedure for the joint minimization of the original non-smoothed objective function in the estimation problem. Here we propose an alternative computational approach, which can be efficiently and stably implemented for solving the problem (17).

Our computation algorithm builds on the following iterative scheme. Let $\hat{\boldsymbol{\theta}}_{1}^{(k)}$ and $\hat{\boldsymbol{\theta}}_{2}^{(k)}$ denote the computed estimates of $\boldsymbol{\theta}_{1}$ and $\boldsymbol{\theta}_{2}$ at the $k$-th iteration step. Note that, fixing the value of $\boldsymbol{\theta}_{1}$ at $\hat{\boldsymbol{\theta}}_{1}^{(k)}$, minimization of the objective function of 17 with respect to $\boldsymbol{\theta}_{2}$ is a smooth optimization problem, which can be easily solved through commonly used numerical optimization solvers (e.g., the optim function of R). On the other hand, fixing the value of $\boldsymbol{\theta}_{2}$ at $\hat{\boldsymbol{\theta}}_{2}^{(k)}$, minimization of the objective function of (17) with respect to $\boldsymbol{\theta}_{1}$ reduces to the following weighted quantile regression problem

$$
\min _{\boldsymbol{\theta}_{1}} \frac{1}{n} \sum_{i=1}^{n} \hat{\xi}_{i}^{(k)}\left[\tau-1\left\{Y_{i} \leq q_{i}\left(\boldsymbol{\theta}_{1}\right)\right\}\right]\left[Y_{i}-q_{i}\left(\boldsymbol{\theta}_{1}\right)\right],
$$

where the weight

$$
\hat{\xi}_{i}^{(k)}=\tilde{K}_{i} \frac{\exp \left[e_{i}\left(\hat{\boldsymbol{\theta}}_{2}^{(k)}\right)\right]}{1+\exp \left[e_{i}\left(\hat{\boldsymbol{\theta}}_{2}^{(k)}\right)\right]}
$$

is nonnegative. The problem (18) can be solved efficiently using well-known algorithms for quantile regression estimation (e.g., the quantreg package of $\mathrm{R}$ ).

Notice that in practice, $\hat{\boldsymbol{\theta}}_{1}^{(1)}$ used for the first iteration step can be obtained from directly solv- 
ing a weighted quantile regression estimation problem with the weight $\hat{\tilde{K}}_{i}$. Using the arguments above, our estimators $\hat{\boldsymbol{\theta}}_{1, \tau}$ and $\hat{\boldsymbol{\theta}}_{2, \tau}$ are thus computed as the solution upon convergence of the iterative algorithm, which proceeds as follows.

For $k \geq 1$,

1. Given $\hat{\boldsymbol{\theta}}_{1}^{(k)}$, compute

$$
\hat{\boldsymbol{\theta}}_{2}^{(k)}=\underset{\boldsymbol{\theta}_{2}}{\arg \min } \frac{1}{n} \sum_{i=1}^{n} \tilde{K}_{i} F Z_{\tau}^{s p}\left(q_{i}\left(\hat{\boldsymbol{\theta}}_{1}^{(k)}\right), e_{i}\left(\boldsymbol{\theta}_{2}\right), Y_{i}\right) .
$$

2. Given $\hat{\boldsymbol{\theta}}_{2}^{(k)}$, compute $\hat{\xi}_{i}^{k}$ in 19 and then compute

$$
\hat{\boldsymbol{\theta}}_{1}^{(k+1)}=\underset{\boldsymbol{\theta}_{1}}{\arg \min } \frac{1}{n} \sum_{i=1}^{n} \hat{\xi}_{i}^{(k)}\left[\tau-1\left\{Y_{i} \leq q_{i}\left(\boldsymbol{\theta}_{1}\right)\right\}\right]\left[Y_{i}-q_{i}\left(\boldsymbol{\theta}_{1}\right)\right] .
$$

Iterate until the solution sequence $\left(\hat{\boldsymbol{\theta}}_{1}^{(k)}, \hat{\boldsymbol{\theta}}_{2}^{(k)}\right)$ converges.

\subsection{Inter-quantile average treatment effect and the Lorenz effect}

We conclude Section 2 by remarking here on two related causal parameters that build upon the CTATE. Define inter-quantile expectation (IQE) of a potential outcome $Y_{d}$ between quantile levels $\tau^{\prime}$ and $\tau$ with $0 \leq \tau^{\prime}<\tau \leq 1$ as

$$
\begin{aligned}
\operatorname{IQE}\left(\tau^{\prime}, \tau, d\right) & :=E\left[Y_{d} \mid Q_{Y_{d} \mid X}\left(\tau^{\prime}\right) \leq Y_{d} \leq Q_{Y_{d} \mid X}(\tau), X\right] \\
& =\frac{\tau C T E_{Y_{d} \mid X}(\tau)-\tau^{\prime} C T E_{Y_{d} \mid X}\left(\tau^{\prime}\right)}{\tau-\tau^{\prime}} .
\end{aligned}
$$

Using the notion of IQE, we can define the inter-quantile average treatment effect (IQATE), which is the difference between inter-quantile expectations of two potential outcomes:

$$
\begin{aligned}
\operatorname{IQATE}\left(\tau^{\prime}, \tau, d, d^{\prime}\right) & :=\operatorname{IQE}\left(\tau^{\prime}, \tau, d\right)-\operatorname{IQE}\left(\tau^{\prime}, \tau, d^{\prime}\right) \\
& =\frac{\tau C T A T E\left(\tau ; d, d^{\prime}\right)-\tau^{\prime} C T A T E\left(\tau^{\prime} ; d, d^{\prime}\right)}{\tau-\tau^{\prime}} .
\end{aligned}
$$

Through (4), the IQATE can be viewed as an aggregate of quantile treatment effects over a range of quantile levels and would thus be useful for providing a summary of heterogeneous treatment effects locally over a specified quantile range. Under Assumption 1 and model (7), we can deduce from (20) that the complier IQATE in the binary treatment causal framework of Section 2.2 reduces to

$$
\frac{\tau \alpha_{2, \tau}-\tau^{\prime} \alpha_{2, \tau^{\prime}}}{\tau-\tau^{\prime}}
$$

which can be readily estimated using the proposed CTATE estimator. 
Conditional tail expectations of potential outcomes $C T E_{Y_{d} \mid X}(\tau)$ are also relevant for deriving the Lorenz curves of the corresponding distributions. Suppose the potential outcome $Y_{d}$ has nonzero mean conditional on $X$. The Lorenz curve, an often used measure for degree of income or wealth inequality, is defined as a ratio of partial mean to the overall mean of $Y_{d}$ :

$$
L O(\tau, d):=\frac{\tau C T E_{Y_{d} \mid X}(\tau)}{E\left[Y_{d} \mid X\right]}=\frac{\tau C T E_{Y_{d} \mid X}(\tau)}{\lim _{\tau \rightarrow 1} \tau C T E_{Y_{d} \mid X}(\tau)} .
$$

The Lorenz effect (Chernozhukov et al., 2013) is then defined as

$$
L O(\tau, d)-L O\left(\tau, d^{\prime}\right)
$$

which is useful on measuring how the degree of inequality of an outcome of interest in a population changes across two treatment regimes. The Lorenz effect for the complier group can also be calculated empirically using the estimated parameters of model (7) in the causal effect framework of this paper.

\section{Asymptotic Result}

In this section we establish asymptotic properties of the estimators $\hat{\boldsymbol{\theta}}_{1, \tau}$ and $\hat{\boldsymbol{\theta}}_{2, \tau}$. We assume that the probability $\pi(X)$ in 10 depends on some parameters $\gamma_{0}$ and rewrite it as $\pi\left(X, \gamma_{0}\right)$. Let $W:=\left(D, X^{\top}\right)^{\top}, V:=(Y, W)$ and $v_{0}(V):=E[Z \mid V]$. Let $\mathcal{V}$ denote the support of $V$ and $\Theta$ be the parameter space of $\left(\boldsymbol{\theta}_{1, \tau}, \boldsymbol{\theta}_{2, \tau}\right)$. Define

$$
\begin{aligned}
K(W, Z ; \gamma) & :=1-\frac{D(1-Z)}{1-\pi(X, \gamma)}-\frac{(1-D) Z}{\pi(X, \gamma)} \\
\bar{K}(V ; v, \gamma) & :=1-\frac{D(1-v(V))}{1-\pi(X, \gamma)}-\frac{(1-D) v(V)}{\pi(X, \gamma)} \\
\tilde{K}(V ; v, \gamma) & :=\min \{\max \{\bar{K}(V ; v, \gamma), 0\}, 1\}
\end{aligned}
$$

With (21) to (23), it can be seen that $K(D, Z, X)$ in (9) can be expressed as $K\left(W, Z ; \gamma_{0}\right)$, and $\bar{K}(Y, D, X)$ in 11 can be expressed as $\bar{K}\left(V ; v_{0}, \gamma_{0}\right)$. The truncated version of $\bar{K}(Y, D, X)$ is then given by $\tilde{K}\left(V ; v_{0}, \gamma_{0}\right)$.

In the following, we derive asymptotic results for a more general setup where $Q_{Y_{i} \mid W_{i}, T=c}(\tau)$ and $C T E_{Y_{i} \mid W_{i}, T=c}(\tau)$ take parametric structural forms $q_{i}\left(\boldsymbol{\theta}_{1}\right):=q\left(W_{i}, \boldsymbol{\theta}_{1}\right)$ and $e_{i}\left(\boldsymbol{\theta}_{2}\right):=e\left(W_{i}, \boldsymbol{\theta}_{2}\right)$, which could be nonlinear yet are known up to some finite dimensional vectors of parameters. Let $\hat{\gamma}$ and $\hat{v}$ denote some estimators of $\boldsymbol{\gamma}_{0}$. We are interested in the parameter values $\boldsymbol{\theta}_{1, \tau}$ and $\boldsymbol{\theta}_{2, \tau}$, which are estimated by $\hat{\boldsymbol{\theta}}_{1, \tau}$ and $\hat{\boldsymbol{\theta}}_{2, \tau}$, where

$$
\left(\hat{\boldsymbol{\theta}}_{1, \tau}, \hat{\boldsymbol{\theta}}_{2, \tau}\right)=\arg \min _{\left(\boldsymbol{\theta}_{1}, \boldsymbol{\theta}_{2}\right) \in \boldsymbol{\Theta}} \frac{1}{n} \sum_{i=1}^{n} \tilde{K}\left(V_{i} ; \hat{v}, \hat{\gamma}\right) F Z_{\tau}\left(q_{i}\left(\boldsymbol{\theta}_{1}\right), e_{i}\left(\boldsymbol{\theta}_{2}\right), Y_{i}\right),
$$

and

$$
\left(\boldsymbol{\theta}_{1, \tau}, \boldsymbol{\theta}_{2, \tau}\right)=\arg \min _{\left(\boldsymbol{\theta}_{1}, \boldsymbol{\theta}_{2}\right) \in \boldsymbol{\Theta}} E\left[F Z_{\tau}\left(q\left(W, \boldsymbol{\theta}_{1}\right), e\left(W, \boldsymbol{\theta}_{2}\right), Y\right) \mid T=c\right] .
$$


We make the following regularity assumptions for consistency of the parameter estimators.

\section{Assumption 2}

1. The data $\left(Y_{i}, D_{i}, X_{i}, Z_{i}\right), i=1, \ldots, n$, are i.i.d..

2. The distribution of $Y$ conditional on $W$ and $T=c$ is absolutely continuous with respect to the Lebesgue measure.

3. The parameter space $\boldsymbol{\Theta}$ is compact.

4. $q\left(W, \boldsymbol{\theta}_{1}\right)$ and $e\left(W, \boldsymbol{\theta}_{2}\right)$ are continuous in $\boldsymbol{\theta}_{1}$ and $\boldsymbol{\theta}_{2}$ respectively for every $\left(\boldsymbol{\theta}_{1}, \boldsymbol{\theta}_{2}\right) \in \mathbf{\Theta}$.

5. The components $G_{1}(),. G_{2}($.$) and G_{2}^{\prime}($.$) in (14) are continuous differentiable functions with$ $G_{1}^{\prime}() \geq 0,. G_{2}^{\prime}()>$.0 and $G_{2}^{\prime \prime}()>$.0 .

6. $P\left(\left\{q\left(W, \boldsymbol{\theta}_{1}\right)=q\left(W, \boldsymbol{\theta}_{1, \tau}\right)\right\} \cap\left\{e\left(W, \boldsymbol{\theta}_{2}\right)=e\left(W, \boldsymbol{\theta}_{2, \tau}\right)\right\} \mid T=c\right)=1$ implies $\left(\boldsymbol{\theta}_{1}, \boldsymbol{\theta}_{2}\right)=\left(\boldsymbol{\theta}_{1, \tau}, \boldsymbol{\theta}_{2, \tau}\right)$.

7. There is a function $b(V)$ with $E[b(V)]<\infty$ such that $b(V)>\left|F Z_{\tau}\left(q\left(W, \boldsymbol{\theta}_{1}\right), e\left(W, \boldsymbol{\theta}_{2}\right), Y\right)\right|$ for all $\left(\boldsymbol{\theta}_{1}, \boldsymbol{\theta}_{2}\right) \in \boldsymbol{\Theta}$.

8. The estimated weight $\bar{K}(V ; \hat{\gamma}, \hat{v})$ is uniformly consistent such that

$$
\sup _{V \in \mathcal{V}}\left|\bar{K}(V ; \hat{\gamma}, \hat{v})-\bar{K}\left(V ; \gamma_{0}, v_{0}\right)\right|=o_{p}(1)
$$

Assumptions 2.1, 2.3 and 2.4 are standard. Assumption 2.2 is sufficient for uniqueness of any $\tau$-quantile of the distribution of $Y$ given $W$ and $T=c$. Assumption 2.5 requires that the FZ loss function (14) be strictly consistent for eliciting quantiles and conditional tail expectations. Assumption 2.6 is a rank condition for parameter identification. For models (6) and (7), this condition immediately holds when, conditional on $T=c$, the support of $W$ is not contained in any proper linear subspace of $\mathbb{R}^{k}$ where $k$ denotes the dimension of $W$. Assumptions 2.5 and 2.6 ensure that the solution $\left(\boldsymbol{\theta}_{1, \tau}, \boldsymbol{\theta}_{2, \tau}\right)$ is unique in the expected FZ loss minimization problem (25). Assumptions 2.7 and 2.8 are imposed for establishing uniform convergence of the empirical objective function in (24) to its population counterpart. Assumption 2.7 is a dominance condition, which can hold under compactness of $\Theta$ and the aforementioned requirements for the FZ loss function, provided that $W$ has a bounded support. Assumption 2.8 hinges on consistency of the plug-in estimators $\hat{\gamma}$ and $\hat{v}$. With these Assumptions 1 and 2, we can have following result.

Theorem 1 If Assumptions 1 and 2 hold, then, for any given $\tau \in(0,1)$,

$$
\left(\hat{\boldsymbol{\theta}}_{1, \tau}, \hat{\boldsymbol{\theta}}_{2, \tau}\right) \stackrel{p}{\rightarrow}\left(\boldsymbol{\theta}_{1, \tau}, \boldsymbol{\theta}_{2, \tau}\right)
$$

In our numerical studies, we estimate the function $v_{0}$ using the power series estimator Newey, 1997) and $\gamma_{0}$ with some parametric binary choice model. Let $W=\left(W_{d}, W_{c}\right)$, where $W_{d}$ and $W_{c}$ denote the discrete and continuous components of $W$ respectively. Let $\mathcal{W}_{d}$ denote the support of $W_{d}$, which takes only finitely many possible values. The estimator $\hat{v}(V)$ takes the form

$$
\hat{v}(V):=\sum_{m \in \mathcal{W}_{d}} 1\left\{W_{d}=m\right\} \hat{v}_{m}\left(Y, W_{c}\right)
$$


where, for each $m \in \mathcal{W}_{d}, \hat{v}_{m}\left(Y, W_{c}\right)$ is a power series estimator of the conditional expectation $v_{0, m}\left(Y, W_{c}\right):=E\left(Z \mid Y, W_{d}=m, W_{c}\right)$. Let $\kappa_{m}$ denote the number of series terms in the power series approximation of $v_{0, m}, s_{m}$ be the order of continuous derivatives of $v_{0, m}$ and $r$ be the dimension of $W_{c}$. The next assumption allows us to verify uniform consistency of the estimated weight $\hat{\bar{K}}($.$) .$

\section{Assumption 3}

1. The support of $\left(Y, W_{c}\right)$ conditional on $W_{d}$ is a Cartesian product of compact connected intervals on which $\left(Y, W_{c}\right)$ has a probability density function that is bounded away from zero.

2. $v_{0, m}\left(Y, W_{c}\right)$ is continuously differentiable of order $s_{m}$ on the support of $\left(Y, W_{c}\right)$.

3. There is a constant $\varepsilon>0$ such that, for the neighborhood $\left\|\gamma-\gamma_{0}\right\| \leq \varepsilon, \pi(x, \gamma)$ is bounded away from 0 and 1 and has a partial derivative $\nabla_{\gamma} \pi(x, \gamma)$, which is uniformly bounded for $\gamma$ in this neighborhood and for $x$ over the support of $X$.

4. $\hat{\gamma} \stackrel{p}{\rightarrow} \gamma_{0}$.

Assumptions 3.1 and 3.2 are standard conditions for power series estimators (Newey, 1997). Assumptions 3.3 is also a mild condition on the smoothness of $\pi(X, \gamma)$. Assumptions 3.4 requires that the estimator $\hat{\gamma}$ be consistent for $\gamma_{0}$. Under Assumption 3, we have the following result.

Lemma 1 Suppose Assumptions 2.1, 2.3 and 3 hold. Then Assumption 2.8 also holds under the series approximation conditions that $r+1<s_{m}, \kappa_{m} \rightarrow \infty$ and $\kappa_{m}^{3} / n \rightarrow 0$ for each $m \in \mathcal{W}_{d}$.

We now provide further regularity assumptions for deriving the asymptotic distribution of the estimators.

\section{Assumption 4}

1. $\hat{\gamma}$ is asymptotically linear with influence function $\psi(X)$ :

$$
\sqrt{n}\left(\hat{\gamma}-\gamma_{0}\right)=\frac{1}{\sqrt{n}} \sum_{i=1}^{n} \psi\left(X_{i}\right)+o_{p}(1),
$$

where $E[\psi(X)]=0$ and $E|\psi(X)|^{2}<\infty$.

2. $E\left[\left(A_{k, \tau}(V)\right)^{2} \mid T=c\right]<\infty$, where $A_{k, \tau}(V), k=1, \ldots, 5$ are the terms defined in Lemma 2 in Appendix A.3.

3. The matrix

$$
\nabla_{\boldsymbol{\theta} \boldsymbol{\theta}} E\left[F Z_{\tau}\left(q\left(W, \boldsymbol{\theta}_{1, \tau}\right), e\left(W, \boldsymbol{\theta}_{2, \tau}\right), Y\right) \mid T=c\right]
$$

is nonsingular, where $\boldsymbol{\theta}:=\left(\boldsymbol{\theta}_{1}, \boldsymbol{\theta}_{2}\right)$.

4. The conditional density $f_{Y \mid W, T=c}\left(q\left(W, \boldsymbol{\theta}_{1, \tau}\right)\right)$ is bounded away from 0 over the support of $W$.

5. The estimated weight $\bar{K}(V ; \hat{\gamma}, \hat{v})$ satisfies that

$$
\sup _{V \in \mathcal{V}}\left|\bar{K}(V ; \hat{\gamma}, \hat{v})-\bar{K}\left(V ; \gamma_{0}, v_{0}\right)\right|=o_{p}\left(n^{-1 / 4}\right) \text {. }
$$


Assumption 4.1 can be easily fulfilled for various parametric estimators in econometrics. Assumption 4.2 is a technical condition related to local Lipschitz continuity of the FZ loss on the neighborhood of true parameter value. Assumptions 4.3 and 4.4 are for the existence of the asymptotic covariance matrix of our estimator. Assumption 4.5, which strengthens Assumption 2.8 , requires that $\bar{K}(V ; \hat{v}, \hat{\gamma})$ should converge uniformly at a rate faster than $n^{-1 / 4}$. For the power series estimator (26), this assumption holds under conditions of Lemma 1 with the growth rate of $\kappa_{m}$ being further restricted such that $\kappa_{m}^{6} / n \rightarrow 0$ and $n^{1 / 4} \kappa_{m}^{1-s_{m} /(r+1)} \rightarrow 0$.

The next theorem establishes asymptotic normality of our proposed estimator and provides the form of its asymptotic covariance matrix.

Theorem 2 If Assumptions 1, 2, and 4 hold, then

$$
\sqrt{n}\left(\left(\hat{\boldsymbol{\theta}}_{1, \tau}, \hat{\boldsymbol{\theta}}_{2, \tau}\right)-\left(\boldsymbol{\theta}_{1, \tau}, \boldsymbol{\theta}_{2, \tau}\right)\right) \stackrel{d}{\rightarrow} N\left(\mathbf{0}, \mathbf{H}_{\tau}^{-1} \boldsymbol{\Omega}_{\tau} \mathbf{H}_{\tau}^{-1}\right)
$$

where

$$
\begin{aligned}
\mathbf{H}_{\tau} & =\nabla_{\boldsymbol{\theta} \boldsymbol{\theta}} E\left[F Z_{\tau}\left(q\left(W, \boldsymbol{\theta}_{1, \tau}\right), e\left(W, \boldsymbol{\theta}_{2, \tau}\right), Y\right) \mid T=c\right] \times P(T=c), \\
\mathbf{\Omega}_{\tau} & =E\left[\mathbf{J}_{\tau} \mathbf{J}_{\tau}^{\top}\right], \\
\mathbf{J}_{\tau} & =K\left(W, Z ; \boldsymbol{\gamma}_{0}\right) \nabla_{\boldsymbol{\theta}} F Z_{\tau}\left(q\left(W, \boldsymbol{\theta}_{1, \tau}\right), e\left(W, \boldsymbol{\theta}_{2, \tau}\right), Y\right)+\mathbf{M}_{\tau} \psi(X), \\
\mathbf{M}_{\tau} & =E\left[\nabla_{\boldsymbol{\theta}} F Z_{\tau}\left(q\left(W, \boldsymbol{\theta}_{1, \tau}\right), e\left(W, \boldsymbol{\theta}_{2, \tau}\right), Y\right)\left[\nabla_{\boldsymbol{\gamma}} K\left(W, Z ; \boldsymbol{\gamma}_{0}\right)\right]^{\top}\right] .
\end{aligned}
$$

For the asymptotic covariance matrix of the estimators, the effect of the presence of the estimated parameter $\hat{\gamma}$ is taken into account. Ignoring the effect of the estimated nuisance parameters will cause the asymptotic covariance matrix inconsistent and lead to invalid confidence interval constructions (Newey and McFadden, 1994). Without such an effect, the vector $\mathbf{J}_{\tau}$ will only have the first term. For estimating the asymptotic covariance matrix, we can use a plug-in estimator by replacing $\left(\boldsymbol{\theta}_{1, \tau}, \boldsymbol{\theta}_{2, \tau}, v_{0}, \gamma_{0}\right)$ in the formula of the asymptotic covariance matrix with their estimates $\left(\hat{\boldsymbol{\theta}}_{1, \tau}, \hat{\boldsymbol{\theta}}_{2, \tau}, \hat{v}, \hat{\boldsymbol{\gamma}}\right)$. In Appendix A.1, we detail the estimation procedures and derive the asymptotic result of the plug-in estimator when $F Z_{\tau}^{s p}(q, e, y)$ is used and the structural forms of $q\left(W, \boldsymbol{\theta}_{1, \tau}\right)$ and $e\left(W, \boldsymbol{\theta}_{2, \tau}\right)$ are linear in parameters. The resulting estimated asymptotic covariance matrix is then used for constructing the pointwise confidence bands of the estimated CTATE and QTE in the empirical application in Section 5.

The above theoretical results of the proposed estimator can immediately be used to derive relevant theoretical properties for estimating the IQATE of (20). Again, we focus on the case when the models are linear in parameters. Divide the interval $(0,1)$ into $L+1$ subintervals with $L$ break points $\tau_{1}, \ldots, \tau_{L}$, where $0<\tau_{1}<\tau_{2} \ldots \tau_{L}<1$. For $l, l^{\prime} \in\{1, \ldots, L\}$, and $l^{\prime}<l$, the IQATE between quantile levels $\tau_{l^{\prime}}$ and $\tau_{l}$ is

$$
\operatorname{IQATE}\left(\tau_{l}, \tau_{l^{\prime}}\right)=\frac{\tau_{l} \alpha_{2, \tau_{l}}-\tau_{l^{\prime}} \alpha_{2, \tau_{l^{\prime}}}}{\tau_{l}-\tau_{l^{\prime}}}
$$


If Assumptions 1, 2 and 4 hold, using Theorem 1 we can obtain the pointwise consistency:

$$
\frac{\tau_{l} \hat{\alpha}_{2, \tau_{l}}-\tau_{l^{\prime}} \hat{\alpha}_{2, \tau_{l^{\prime}}}}{\tau_{l}-\tau_{l^{\prime}}} \stackrel{p}{\rightarrow} \frac{\tau_{l} \alpha_{2, \tau_{l}}-\tau_{l^{\prime}} \alpha_{2, \tau_{l^{\prime}}}}{\tau_{l}-\tau_{l^{\prime}}}
$$

The standard deviation of the above estimator for IQATE with $\hat{\alpha}_{2, \tau_{l}}$ and $\hat{\alpha}_{2, \tau_{l^{\prime}}}$ is given by

$$
\sqrt{\frac{1}{\left(\tau_{l}-\tau_{l^{\prime}}\right)^{2}}\left[\tau_{l}^{2} \operatorname{Var}\left(\hat{\alpha}_{2, \tau_{l}}\right)+\tau_{l^{\prime}}^{2} \operatorname{Var}\left(\hat{\alpha}_{2, \tau_{l^{\prime}}}\right)-2 \tau_{l} \tau_{l^{\prime}} \operatorname{Cov}\left(\hat{\alpha}_{2, \tau_{l}}, \hat{\alpha}_{2, \tau_{l^{\prime}}}\right)\right]}
$$

If $\operatorname{dim}(X)=p \times 1, \operatorname{Var}\left(\hat{\alpha}_{2, \tau_{l}}\right)$ (and $\operatorname{Var}\left(\hat{\alpha}_{2, \tau_{l}^{\prime}}\right)$ ) is the $(p+2)$ th diagonal element of the matrix $\mathbf{H}_{\tau_{l}}^{-1} \boldsymbol{\Omega}_{\tau_{l}} \mathbf{H}_{\tau_{l}}^{-1}$ (and $\left(\mathbf{H}_{\tau_{l^{\prime}}}^{-1} \boldsymbol{\Omega}_{\tau_{l^{\prime}}} \mathbf{H}_{\tau_{l^{\prime}}}^{-1}\right)$ ), and $\operatorname{Cov}\left(\hat{\alpha}_{2, \tau_{l}}, \hat{\alpha}_{2, \tau_{l^{\prime}}}\right)$ is the $(p+2)$ th diagonal element of the matrix $\mathbf{H}_{\tau_{l}}^{-1} E\left[\mathbf{J}_{\tau_{l}} \mathbf{J}_{\tau_{l^{\prime}}}^{\top}\right] \mathbf{H}_{\tau_{l^{\prime}}}^{-1}$, both scaled by $n^{-1}$.

Next we provide result of uniform consistency: $\left(\hat{\boldsymbol{\theta}}_{1, \tau}, \hat{\boldsymbol{\theta}}_{2, \tau}\right) \stackrel{p}{\rightarrow}\left(\boldsymbol{\theta}_{1, \tau}, \boldsymbol{\theta}_{2, \tau}\right)$ uniformly over $\tau \in \mathcal{T} \subset(0,1)$. We focus on the special case when the loss function $F Z_{\tau}^{s p}(q, e, y)$ is used. Let

$$
h(\tau, \boldsymbol{\theta}):=\tau\left[F Z_{\tau}^{s p}\left(q\left(W, \boldsymbol{\theta}_{1}\right), e\left(W, \boldsymbol{\theta}_{2}\right), Y\right)-\ln (1+\exp (Y))\right],
$$

where $\boldsymbol{\theta}:=\left(\boldsymbol{\theta}_{1}, \boldsymbol{\theta}_{2}\right)$. Notice that minimizing $E\left[F Z_{\tau}^{s p}\left(q\left(W, \boldsymbol{\theta}_{1}\right), e\left(W, \boldsymbol{\theta}_{2}\right), Y\right)\right]$ and $E[h(\tau, \boldsymbol{\theta})]$ w.r.t. $\boldsymbol{\theta}$ will obtain the same minimizer, since the former is just the latter scaled by a positive and finite constant $1 / \tau$ and plus $E[\ln (1+\exp (Y))]$. Therefore using $h(\tau, \boldsymbol{\theta})$ as a loss function to estimate $\boldsymbol{\theta}$ will yield the same result as using the $F Z_{\tau}^{s p}\left(q\left(w, \boldsymbol{\theta}_{1}\right), e\left(w, \boldsymbol{\theta}_{2}\right), y\right)$. We will use $h(\tau, \boldsymbol{\theta})$ as the loss function in proving the uniform consistency. The proof will rely on using the following additional assumptions and results of Newey (1991) to show that the empirical loss function uniformly converges to the true loss function over $(\tau, \boldsymbol{\theta}) \in \mathcal{T} \times \boldsymbol{\Theta}$.

\section{Assumption 5}

1. $\mathcal{T}$ is a closed sub-interval of $(0,1)$.

2. The partial derivative functions $\nabla_{\boldsymbol{\theta}} E[h(\tau, \boldsymbol{\theta}) \mid T=c]$ and $\frac{\partial}{\partial \tau} E[h(\tau, \boldsymbol{\theta}) \mid T=c]$ are both bounded for all $(\tau, \boldsymbol{\theta}) \in \mathcal{T} \times \boldsymbol{\Theta}$.

3. $E\left[B_{k}(V) \mid T=c\right]<\infty$, where $B_{k}(V) \geq 0, k=1, \ldots, 5$ are the terms defined in Lemma 2 in Appendix A.4.

Assumption 5.1 is standard. Assumption 5.2 is for the equicontinuity of the true loss function $E\left[\bar{K}\left(V ; v_{0}, \gamma_{0}\right) h(\tau, \boldsymbol{\theta})\right]$. Assumptions 5.3 requires the first moment of the quantity $B_{k, \tau}(V), k=$ $1, \ldots, 5$ (defined in Lemma 3 in the Appendix A.4) should be finite. This is a technical condition related to global Lipschitz continuity of the FZ loss on the parameter space. The next theorem establishes uniform consistency of our estimator over the quantile index range $\mathcal{T}$.

Theorem 3 Let the FZ loss in (24) take the form (16). If Assumptions 1, 2 and 5 hold, then

$$
\sup _{\tau \in \mathcal{T}}\left\|\left(\hat{\boldsymbol{\theta}}_{1, \tau}, \hat{\boldsymbol{\theta}}_{2, \tau}\right)-\left(\boldsymbol{\theta}_{1, \tau}, \boldsymbol{\theta}_{2, \tau}\right)\right\| \stackrel{p}{\rightarrow} 0 .
$$




\section{Simulation}

In this section we examine finite-sample performance of our proposed method in the simulations. The data $\left(Y_{i}, D_{i}, Z_{i}, X_{i}\right), i=1, \ldots, n$, where $X_{i}=\left(X_{1 i}, X_{2 i}\right)$, for the simulation are generated according to the following design:

$$
\begin{aligned}
X_{1 i}, X_{2 i} & \stackrel{\text { i.i.d }}{\sim} U(0,1), \\
Z_{i} \mid X_{i} & \stackrel{\text { i.i.d }}{\sim} \operatorname{Bern}\left(\Phi\left(-1+X_{1 i}+X_{2 i}\right)\right), \\
D_{i} & =Z_{i} D_{1 i}+\left(1-Z_{i}\right) D_{0 i}, \\
Y_{i} & =D_{i} Y_{1 i}+\left(1-D_{i}\right) Y_{0 i} .
\end{aligned}
$$

Here $U(0,1)$ is the uniform distribution in $[0,1], \operatorname{Bern}(p)$ is the Bernoulli distributions with parameter $p, \Phi($.$) denotes the cumulative distribution function of the standard normal random$ variable, and

$$
\begin{aligned}
D_{1 i} & =1\left\{\vartheta_{i}>-0.67\right\} \\
D_{0 i} & =1\left\{\vartheta_{i}>0.67\right\} \\
Y_{1 i} & =\left(b_{0}+b_{1}+b_{2} X_{1 i}+b_{3} X_{2 i}\right) \varepsilon_{i}, \\
Y_{0 i} & =\left(b_{1}+b_{2} X_{1 i}+b_{3} X_{2 i}\right) \varepsilon_{i}, \\
\left(\varepsilon_{i}, \vartheta_{i}\right) \mid X_{i} & \stackrel{\text { i.i.d. }}{\sim} M V N\left(\mathbf{0}, \Sigma_{\varepsilon, \vartheta}\right), \Sigma_{\varepsilon, \vartheta}=\left(\begin{array}{cc}
1 & \rho \\
\rho & 1
\end{array}\right),
\end{aligned}
$$

where $\operatorname{MVN}\left(\mathbf{0}, \Sigma_{\varepsilon, \vartheta}\right)$ denotes the multivariate normal distribution with mean zero and covariance matrix $\Sigma_{\varepsilon, \vartheta}$. We set $T_{i}=a$, if $D_{1 i}=D_{0 i}=1 ; T_{i}=c$, if $D_{1 i}=1, D_{0 i}=0 ;$ and $T_{i}=n e$, if $D_{1 i}=D_{0 i}=0$. Under this simulation design, the proportion of compliers is roughly $50 \%$, the proportions of always and never takers are both around $25 \%$, and there is no defier. The correlation coefficient $\rho$ controls the degree of endogeneity. When $\rho \neq 0$, for $T=a$, ne (always and never takers), the treatment status $D$ is correlated with potential outcomes $Y_{1}$ and $Y_{0}$ through $\varepsilon$ and the endogeneity arises. But for $T=c$ (compliers), the condition of unconfounded treatment selection: $D \perp\left(Y_{1}, Y_{0}\right) \mid\left(X_{1}, X_{2}\right)$ holds here.

Under this setting,

$$
\begin{aligned}
Q_{Y \mid D, X, T=c}(\tau) & =\left(b_{0} D+b_{1}+b_{2} X_{1}+b_{3} X_{2}\right) Q_{\varepsilon \mid X, T=c}(\tau), \\
& =\alpha_{1, \tau} D+\beta_{11, \tau}+\beta_{12, \tau} X_{1}+\beta_{13, \tau} X_{2}, \\
C T E_{Y \mid D, X, T=c}(\tau) & =\left(b_{0} D+b_{1}+b_{2} X_{1}+b_{3} X_{2}\right) C T E_{\varepsilon \mid X, T=c}(\tau), \\
& =\alpha_{2, \tau} D+\beta_{21, \tau}+\beta_{22, \tau} X_{1}+\beta_{23, \tau} X_{2} .
\end{aligned}
$$

The complier QTE and CTATE are $\alpha_{1, \tau}:=b_{0} Q_{\varepsilon \mid X, T=c}(\tau)$ and $\alpha_{2, \tau}:=b_{0} C T E_{\varepsilon \mid X, T=c}(\tau)$ respectively. We set parameters $b_{0}=1, b_{1}=0$ and $b_{2}=b_{3}=1$. We consider two different sample sizes $n \in\{500,3000\}$, and the simulation is iterated 1000 times. We perform simulations under the cases of $\rho=0$ (no endogeneity) and $\rho=0.5$ (with endogeneity). 
We consider two alternative constructions of projected weights in the weighted FZ loss minimization approach and assess how they affect finite-sample performance of our proposed method. The first weight estimator is given by

$$
\bar{K}(Y, D, X ; \hat{v}, \hat{\gamma})=1-\frac{D(1-\hat{v}(Y, D, X))}{1-\pi(X, \hat{\gamma})}-\frac{(1-D) \hat{v}(Y, D, X)}{\pi(X, \hat{\gamma})},
$$

where $\hat{v}(Y, D, X)$ is an estimate for $E[Z \mid Y, D, X]$ from a polynomial regression, and $\pi(X, \hat{\gamma})$ is an estimate for $P(Z=1 \mid X)$ from a probit model with an intercept term and covariates $\left(X_{1}, X_{2}\right)$. The estimate $\hat{v}(Y, D, X)$ is computed separately for the $D=1$ and $D=0$ groups, and the covariates used in the regressions include $(Y, X)$, their higher order and interaction terms. For the second weight estimator, we first calculate $K(D, Z, X)$ of $(9)$ for each observation using $\pi(X, \hat{\gamma})$ obtained from the same probit model used in the first estimator. We then fit a polynomial regression of the calculated $K(D, Z, X)$ on $(Y, D, X)$, their higher order and interaction terms and two additional covariates $\left(D_{0}, D_{1}\right)$ which entail crucial information for classifying types of individuals. The fitted value from this polynomial regression is used as the second projected weight estimator. By the law of iterated expectations, the identity 12 still holds with the weight function $\bar{K}(Y, D, X)$ being replaced by the weight $\bar{K}_{2}\left(Y, D, X, D_{1}, D_{0}\right):=E\left[K(D, Z, X) \mid Y, D, X, D_{1}, D_{0}\right]$, which motivates our construction of the second type of projected weight. Adopting proof of Lemma 3.2 of Abadie et al. (2002), we can see that $\bar{K}_{2}\left(Y, D, X, D_{1}, D_{0}\right)=P\left(T=c \mid Y, D, X, D_{1}, D_{0}\right)$. Thus working with the weight $\bar{K}_{2}$ amounts to estimating with information on classifying types of individuals. We note that this weight estimator is infeasible in practice as we do not observe both $D_{0}$ and $D_{1}$ for each individual in the data. Yet, we compare the results using $\sqrt{28}$ with those of $\bar{K}_{2}$ as the latter exploits more information and is expected to improve performances of the resulting weighted FZ loss minimization estimators. Finally, since the weights are bounded from zero and one, if the estimated weights are greater than one or less than zero, we will shrink their values to 1 or 0 .

Let M1 and M2 denote the weighted FZ loss minimization estimation approaches implemented with the aforementioned first and second projected weight estimators respectively. In addition, we also consider the following two benchmarks where no weighting scheme is used: (1) Estimation using all data without imposing any weight on the samples (no adjustment for endogeneity, denoted by M3); (2) Estimation using only the sample of compliers (oracle estimation, denoted by M4).

Figures 1 to 4 summarize the simulation results, which include the biases, variances and mean squared errors (MSEs) of the CTATE and QTE estimators under the settings M1 to M4. The key findings are discussed as follows. For $\rho=0$, there is no concern of endogeneity. In this case, the CTATE and QTE, which were estimated using all data without imposing any weight scheme (M3), had the lowest variance and MSE. Imposing weights to account for endogeneity would increase estimator variability, as can be seen from our theoretical result in Theorem 2 that the estimated weight contributes to variances of the proposed estimators. The estimation results from using only the complier samples (M4) were associated with a higher variance than those of M3. This is mainly due to the sample size effect as the approach M4 tends to use fewer observations than M3 does in the implementation. The profiles of variances for the estimated 
CTATE reveal a downward trend as the quantile level rises, but those for the estimated QTE indicate that the variances were larger at the lower and higher quantile levels. For the estimation bias, the simulation results are mixed and there is no clear dominant method here. The bias, variance and MSE were all improved as the sample size increased from 500 to 3000.

For $\rho=0.5$, the endogeneity issue arises. In this case, M3 results in much higher bias and MSE than the other three methods, although it still results in a lower variance. The high MSE of M3 is mostly due to the high bias from no adjustment for endogeneity. The reasons for the lower variance of M3 are the same as those in the case of $\rho=0$ : all samples are used in the estimations and there is no estimated weight. M4 (the oracle estimation case) was associated with the lowest bias, as it is the one using only the sample of compliers. The bias curve under M2 was flat over the quantile levels, while that under M1 indicated some declining trend. It is worth noting that M1 resulted in a higher MSE than M2, which suggests that using potential treatment status information for estimating the weight may be helpful on improving the MSE. The performances for M1, M2 and M4 overall became evidently better when the sample size increased. In sum, in the case of endogeneity, although estimation without adjustment for endogeneity (M3) could still yield a lower variance, it could also result in a higher bias and thus a higher MSE. The higher variances under M1 and M2 arise from using estimated weights. Yet, these weight schemes help to effectively mitigate the estimation bias. On the whole, relatively to the M3 case, the overall MSE under M1 and M2 can get substantially improved under the weighting adjustment for endogeneity.

\section{Empirical Application: Effects of Enrolling in JTPA Services on Earnings}

In the following we illustrate usefulness of our proposed method in an empirical study. We estimate the CTATE using our proposed weighted FZ loss minimization approach to evaluate effects of enrolling in Title II programs of the Job Training Partnership Act (JTPA) in the US. We use the data of adult men and women who participated in these programs between November 1987 and September 1989. These data were previously used in Abadie et al. (2002) for estimating the QTE on earnings of the job training programs. The outcome variables $Y$ are 30-month earnings of the adult men and women. The treatment variable $D$ is a binary variable for enrollment in the JTPA services (1) or not (0), and the instrument variable $Z$ is a binary variable for being offered such services (1) or not (0). The exogenous covariates include age, which is a categorical variable, as well as a set of dummy variables: black, Hispanic, high-school graduates (including the GED holders), martial status, AFDC receipt (for adult women) and whether the applicant worked at least 12 weeks in the 12 months preceding random assignment, the original recommended service strategy (classroom, OJT/JSA, other) and whether earnings data are from the second follow-up survey. The total samples size is 11,204 (5,102 for adult men and 6,102 for adult women).

Offers of the JTPA services were randomly assigned to applicants but only about 60 percent of those who were offered the services enrolled in the programs (Abadie et al. 2002). This may induce the problem of endogeneity that the treatment status may be self-selected and correlated with the 
potential outcomes. Since the offers were randomly assigned and were considered to potentially affect an applicant's intention of participating in the program, we use the offer assignment as an instrument variable. We estimate the models (6) and (7) over a grid of quantile levels $\tau$ ranging from 0.1 to 0.9 with the grid size being 0.01 (81 grid points). We estimate $E[Z \mid Y, D, X]$ with a power series estimator separately for $D=1$ and $D=0$, and include the outcome variable $Y$ and its higher order terms as covariates. The offer assignment probability, $P(Z=1 \mid X)$, is estimated with a probit model. These estimates are then used to construct the weight $\tilde{K}($.$) in$ (23) to account for endogeneity in the estimation problem. In Figure 5 and 6, we present profiles of the estimated QTEs and CTATEs (the parameter estimates $\alpha_{1, \tau}$ of (6) and $\alpha_{2, \tau}$ of (7p) and the corresponding $95 \%$ pointwise confidence band (pcb) values, evaluated with the analytic standard errors of the estimators (see Appendix A.1), over the specified range of quantile levels. We also show the $95 \%$ pointwise and simultaneous confidence band (scb) results from a bootstrap with 300 bootstrap samples. The procedures for conducting the bootstrap and constructing the scb can be found in Appendix A.5.

Figure 5 shows estimation results for the case of adult men. For the estimated CTATEs, they increase monotonically with the quantile levels and those estimated under the assumption of the treatment being endogenous overall have a lower value. For the estimated QTEs, under different assumptions, they have different profile patterns and overall those estimated under the endogenous treatment assumption also have a lower value. The pcb results evaluated with the analytic standard errors and bootstrap are visually not too different. Under the assumption of treatment being exogenous, the scb indicate that the CTATEs are statistically significantly positive over all quantile levels. The QTEs have a similar but slightly weaker result as the scb results cover value of zero at the high quantile levels. Under the endogeneous treatment assumption, however, the evidence of positivity for both the CTATEs and QTEs is much weaker, as the pcb and scb results cover value of zero over almost all quantile levels. In term of the stochastic dominance relation, the results of scb under the exogenous treatment assumption indicate that for the adult men, earnings from not participating the JTPA is second, but not first order stochastically dominated by those from participating the JTPA. But under the endogeneous treatment assumption, both the FSD and SSD of participating the JTPA do not hold.

Figure 6 shows estimation results for the case of adult women. The evidence for positive QTEs and CTATEs shown here is stronger than that for the case of adult men, regardless of the assumption on endogeneity of treatment selection. Under both assumptions, the results of scb indicate that the CTATEs are statistically significantly positive over all quantile levels, although those estimated under the endogeneous treatment assumption have a wider range. The QTEs also exhibit a similar result except at the high quantile levels. In term of the stochastic dominance relation, the scb results indicate that for the adult women, earnings from not participating the JTPA is second, but not first order stochastically dominated by those from participating the JTPA, no matter whether the treatment status is endogenous.

We then divide the quantile levels into eight equal-length intervals and estimate the corresponding inter-quantile average treatment effect (IQATE). We also compare the estimated IQATE with a naive estimator: a local average of the estimated QTE within the same interval of quantile 
levels. Figure 7 shows estimation results of the IQATE, the corresponding pcb results (implemented with the analytic standard errors and the bootstrap) and a local average of the estimated QTE (LAVG-QTE) for earnings of the adult men (upper panel) and adult women (lower) at different intervals of quantile levels.

For the case of adult men, under the assumption of no endogeneity, the IQATEs are statistically significantly positive over the eight intervals of quantile levels. But under the endogeneous treatment assumption, only the IQATEs above the quantile level 0.6 are statistically significantly positive. It is worth noting that under the endogeneous treatment assumption, at the intervals of quantile levels 0.8 and 0.9 , the QTEs are not all statistically significantly positive, but the IQATE is. This indicates that the IQATE might provide a more coherent result when we want to evaluate a policy at the intervals of quantile levels. For the case of adult women, the IQATE is statistically significantly positive over the eight intervals of quantile levels, irrespective of the assumption on treatment endogeneity. Comparing the estimated IQATE and LAVG-QTE, they are not very different when the quantile level is above 0.5 , but at the quantile levels lower than 0.5 , they rather show mild differences in the cases under the endogeneous treatment assumption.

\section{Conclusion}

We have introduced the conditional tail average treatment effect (CTATE), defined as the difference between conditional tail expectations of potential outcomes. The CTATE is a valuable tool for policy evaluations, since it allows for capturing heterogeneity of treatment effects over different quantiles and is useful for detecting the second order stochastic dominance and for estimating the Lorenz curve. We have shown that a semiparametric method with a class of consistent loss functions proposed by Fissler and Ziegel (2016), can be used to estimate the CTATE under endogeneity. We also have derived pointwise asymptotic results for the statistical inference of the estimations. Some uniform asymptotic results are also discussed. Simulation results show that the proposed method works well in the presence of treatment endogeneity. We apply our estimation approach to a policy evaluation for the JTPA program participation. We find that, only in the adult male worker case for which the estimation incorporates weighting adjustment for endogeneity, the CTATE is not statistically positive over all quantile levels. Yet, regardless of the presence of adjustment for endogeneity, our results also indicate that the QTE for both adult male and female workers is not statistically positive across all quantile levels. These empirical results suggest that the job training programs were beneficial for risk averse female workers. 


\section{A Appendix}

To ease the notation, throughout the Appendix, we will omit the random variables in the arguments for the structural functions $q\left(W, \boldsymbol{\theta}_{1, \tau}\right)$ and $e\left(W, \boldsymbol{\theta}_{2, \tau}\right)$ as well as the weights $K(W, Z ; \boldsymbol{\gamma}), \bar{K}(V ; v, \boldsymbol{\gamma})$ and $\tilde{K}(V ; v, \boldsymbol{\gamma})$ and abbreviate them respectively by $q\left(\boldsymbol{\theta}_{1, \tau}\right), e\left(\boldsymbol{\theta}_{2, \tau}\right) K(\boldsymbol{\gamma}), \bar{K}(v, \boldsymbol{\gamma})$ and $\tilde{K}(v, \boldsymbol{\gamma})$. We also let $q_{i}\left(\boldsymbol{\theta}_{1, \tau}\right):=q\left(W_{i}, \boldsymbol{\theta}_{1, \tau}\right), e_{i}\left(\boldsymbol{\theta}_{2, \tau}\right):=e\left(W_{i}, \boldsymbol{\theta}_{2, \tau}\right), K_{i}(\boldsymbol{\gamma}):=K\left(W_{i}, Z_{i} ; \boldsymbol{\gamma}\right), \bar{K}_{i}(v, \boldsymbol{\gamma}):=\bar{K}\left(V_{i} ; v, \boldsymbol{\gamma}\right)$ and $\tilde{K}_{i}(v, \gamma):=\tilde{K}\left(V_{i} ; v, \gamma\right)$.

\section{A.1 Estimating the Asymptotic Covariance Matrix of the Estimators}

For estimating the asymptotic covariance matrix of the estimators, we focus on the case where the function $F Z_{\tau}^{s p}(q, e, y)$ is used and the complier quantile and conditional tail expectation models are linear in parameters. In this setting, $\nabla_{\boldsymbol{\theta}_{1}} q\left(\boldsymbol{\theta}_{1, \tau}\right)=\nabla_{\boldsymbol{\theta}_{2}} e\left(\boldsymbol{\theta}_{2, \tau}\right)=W$, where $W=\left(D, X^{\top}\right)^{\top}$. Recall that

$$
F Z_{\tau}^{s p}(q, e, y)=\frac{\exp (e)}{1+\exp (e)}\left[e+L Q_{\tau}(q, y)\right]+\ln (1+\exp (y))-\ln (1+\exp (e)),
$$

where $L Q_{\tau}(q, y):=\tau^{-1} \max (q-y, 0)-q$. Let $G_{s p}(t):=\ln (1+\exp (t))$ denote the softplus function. Then

$$
G_{s p}^{\prime}(e)=\frac{\exp (e)}{1+\exp (e)}, G_{s p}^{\prime \prime}(e)=\frac{\exp (e)}{[1+\exp (e)]^{2}} .
$$

Notice that both $G_{s p}^{\prime}(e)$ and $G_{s p}^{\prime \prime}(e)$ are bounded and continuous functions of $e$.

Let $\mathbf{J}_{\tau}^{s p}$ denote $\mathbf{J}_{\tau}$ when $F Z_{\tau}^{s p}(q, e, y)$ is used:

$$
\mathbf{J}_{\tau}^{s p}=K\left(\gamma_{0}\right) \nabla_{\boldsymbol{\theta}} F Z_{\tau}^{s p}\left(q\left(\boldsymbol{\theta}_{1, \tau}\right), e\left(\boldsymbol{\theta}_{2, \tau}\right), Y\right)+\mathbf{M}_{\tau} \psi(X),
$$

where

$$
\nabla_{\boldsymbol{\theta}} F Z_{\tau}^{s p}\left(q\left(\boldsymbol{\theta}_{1, \tau}\right), e\left(\boldsymbol{\theta}_{2, \tau}\right), Y\right)=\left(\begin{array}{c}
\frac{1}{\tau}\left(1\left\{Y \leq q\left(\boldsymbol{\theta}_{1, \tau}\right)\right\}-\tau\right) G_{s p}^{\prime}\left(e\left(\boldsymbol{\theta}_{2, \tau}\right)\right) W \\
G_{s p}^{\prime \prime}\left(e\left(\boldsymbol{\theta}_{2, \tau}\right)\right)\left[e\left(\boldsymbol{\theta}_{2, \tau}\right)+L Q_{\tau}\left(q\left(\boldsymbol{\theta}_{1, \tau}\right), Y\right)\right] W
\end{array}\right)
$$

and $\boldsymbol{\Omega}_{\tau}^{s p}=E\left[\mathbf{J}_{\tau}^{s p}\left(\mathbf{J}_{\tau}^{s p}\right)^{\top}\right]$. Note that the function 29 coincides with the derivative of $F Z_{\tau}^{s p}$ with respect to $\boldsymbol{\theta}=\left(\boldsymbol{\theta}_{1}, \boldsymbol{\theta}_{2}\right)$ whenever the latter exists. Let $\mathbf{C}_{\tau, 11}^{s,}, \mathbf{C}_{\tau, 22}^{s p}$ and $\mathbf{H}_{\tau}^{s p}$ denote $\mathbf{C}_{\tau, 11}, \mathbf{C}_{\tau, 22}$ and $\mathbf{H}_{\tau}$ in the proof of Theorem 2 when $F Z_{\tau}^{s p}(q, e, y)$ is used:

$$
\mathbf{H}_{\tau}^{s p}=\left(\begin{array}{cc}
\mathbf{C}_{\tau, 11}^{s p *} & \mathbf{0} \\
\mathbf{0} & \mathbf{C}_{\tau, 22}^{s p *}
\end{array}\right)
$$

where

$$
\begin{aligned}
\mathbf{C}_{\tau, 11}^{s p *} & =\mathbf{C}_{\tau, 11}^{s p} \times P(T=c) \\
& =E\left[\bar{K}\left(v_{0}, \gamma_{0}\right) f_{Y \mid W, T=c}\left(q\left(\boldsymbol{\theta}_{1, \tau}\right)\right) \frac{1}{\tau} G_{s p}^{\prime}\left(e\left(\boldsymbol{\theta}_{2, \tau}\right)\right) W W^{\top}\right], \\
\mathbf{C}_{\tau, 22}^{s p *} & =\mathbf{C}_{\tau, 22}^{s p} \times P(T=c) \\
& =E\left[\bar{K}\left(v_{0}, \gamma_{0}\right) G_{s p}^{\prime \prime}\left(e\left(\boldsymbol{\theta}_{2, \tau}\right)\right) W W^{\top}\right] .
\end{aligned}
$$

The asymptotic covariance matrix of the proposed estimators is $\left(\mathbf{H}_{\tau}^{s p}\right)^{-1} \boldsymbol{\Omega}_{\tau}^{s p}\left(\mathbf{H}_{\tau}^{s p}\right)^{-1}$. To estimate this asymptotic covariance matrix, we use the plug-in estimator: replacing $\left(\boldsymbol{\theta}_{1, \tau}, \boldsymbol{\theta}_{2, \tau}, v_{0}, \boldsymbol{\gamma}_{0}\right)$ in $\left(\mathbf{H}_{\tau}^{s p}\right)^{-1} \boldsymbol{\Omega}_{\tau}^{s p}\left(\mathbf{H}_{\tau}^{s p}\right)^{-1}$ with estimates $\left(\hat{\boldsymbol{\theta}}_{1, \tau}, \hat{\boldsymbol{\theta}}_{2, \tau}, \hat{v}, \hat{\gamma}\right)$ where $\left(\hat{\boldsymbol{\theta}}_{1, \tau}, \hat{\boldsymbol{\theta}}_{2, \tau}\right)$ are obtained from the method introduced in Section 2 
and $(\hat{v}, \hat{\gamma})$ are constructed such that Assumption 4 holds. We first propose using

$$
\hat{\mathbf{\Omega}}_{\tau}^{s p}=\frac{1}{n} \sum_{i=1}^{n} \hat{\mathbf{J}}_{i, \tau}^{s p} \hat{\mathbf{J}}_{i, \tau}^{s p \top}
$$

to estimate $\boldsymbol{\Omega}_{\tau}^{s p}$, where

$$
\hat{\mathbf{J}}_{i, \tau}^{s p}=K_{i}(\hat{\gamma}) \nabla_{\boldsymbol{\theta}} F Z_{\tau}^{s p}\left(q_{i}\left(\hat{\boldsymbol{\theta}}_{1, \tau}\right), e_{i}\left(\hat{\boldsymbol{\theta}}_{2, \tau}\right), Y_{i}\right)+\hat{\mathbf{M}}_{\tau} \hat{\psi}\left(X_{i}\right),
$$

and

$$
\hat{\mathbf{M}}_{\tau}=\frac{1}{n} \sum_{i=1}^{n} \nabla_{\boldsymbol{\theta}} F Z_{\tau}\left(q_{i}\left(\hat{\boldsymbol{\theta}}_{1, \tau}\right), e_{i}\left(\hat{\boldsymbol{\theta}}_{2, \tau}\right), Y_{i}\right) \nabla_{\gamma} K_{i}(\hat{\gamma})
$$

Here $\hat{\psi}\left(X_{i}\right)$ is an estimate of $\psi\left(X_{i}\right)$. For example, if a probit model with a link function $\Phi\left(X_{i}^{\top} \gamma\right)$ is used to estimate $\gamma$,

$$
\psi\left(X_{i}\right)=-\left(\frac{1}{n} \sum_{i=1}^{n} \frac{\partial s\left(\gamma ; X_{i}\right)}{\partial \gamma}\right)^{-1} s\left(\gamma ; X_{i}\right),
$$

where

$$
s\left(\boldsymbol{\gamma} ; X_{i}\right)=\left(\frac{Y_{i} \phi\left(X_{i}^{\top} \gamma\right)}{\Phi\left(X_{i}^{\top} \gamma\right)}-\frac{\left(1-Y_{i}\right) \phi\left(X_{i}^{\top} \gamma\right)}{1-\Phi\left(X_{i}^{\top} \gamma\right)}\right) X_{i}
$$

and $-\left(\frac{1}{n} \sum_{i=1}^{n} \partial s\left(\boldsymbol{\gamma} ; X_{i}\right) / \partial \gamma\right)^{-1}$ is the inverse of the information matrix. $\hat{\psi}\left(X_{i}\right)$ can be constructed by plugging $\hat{\gamma}$ into $s\left(\gamma ; X_{i}\right)$ and replacing $-\left(\frac{1}{n} \sum_{i=1}^{n} \partial s\left(\gamma ; X_{i}\right) / \partial \gamma\right)^{-1}$ in 30 with the estimated covariance matrix of $\hat{\gamma}$ multiplied by the sample size. Let

$$
\varsigma_{\lambda_{n}, i}\left(\hat{\boldsymbol{\theta}}_{1, \tau}\right)=\frac{1\left\{\left|Y_{i}-q_{i}\left(\hat{\boldsymbol{\theta}}_{1, \tau}\right)\right| \leq \lambda_{n}\right\}}{2 \lambda_{n}}
$$

where $\lambda_{n}$ is a deterministic function of $n$ and satisfies certain conditions. We then propose using

$$
\hat{\mathbf{H}}_{\tau}^{s p}=\left(\begin{array}{cc}
\hat{\mathbf{C}}_{\tau, 11}^{s p *} & \mathbf{0} \\
\mathbf{0} & \hat{\mathbf{C}}_{\tau, 22}^{s p *}
\end{array}\right)
$$

to estimate $\mathbf{H}_{\tau}^{s p}$, where

$$
\begin{aligned}
\hat{\mathbf{C}}_{\tau, 11}^{s p *} & =\frac{1}{n} \sum_{i=1}^{n}\left\{\tilde{K}_{i}(\hat{v}, \hat{\gamma}) \varsigma_{\lambda, i}\left(\hat{\boldsymbol{\theta}}_{1, \tau}\right) \frac{1}{\tau} G_{s p}^{\prime}\left(e_{i}\left(\hat{\boldsymbol{\theta}}_{2, \tau}\right)\right) W_{i} W_{i}^{\top}\right\}, \\
\hat{\mathbf{C}}_{\tau, 22}^{s p *} & =\frac{1}{n} \sum_{i=1}^{n} \tilde{K}_{i}(\hat{v}, \hat{\gamma}) G^{\prime \prime}\left(e_{i}\left(\hat{\boldsymbol{\theta}}_{2, \tau}\right)\right) W_{i} W_{i}^{\top} .
\end{aligned}
$$

The estimator $\varsigma_{\lambda_{n}, i}\left(\hat{\boldsymbol{\theta}}_{1, \tau}\right)$ is the Powell sandwich estimator Powell, 1984$)$ and is used to approximate the conditional density $f_{Y \mid W, T=c}\left(q\left(\boldsymbol{\theta}_{1, \tau}\right)\right)$. It was also used in Powell (1984), Engle and Manganelli (2004), Angrist et al. (2006) and Patton et al. (2019). The bandwidth function $\lambda_{n}$ can be set equal to $O\left(n^{-1 / 3}\right)$ (Patton et al. 2019) or chosen with some specific method (see discussions in Koenker (2005)).

Theorem 4 If Assumptions 1, 2 and 4 hold, $E\left[\left\|W W^{T}\right\| \mid T=c\right]<\infty$, and $\lambda_{n}=o(1)$ and $\lambda_{n}^{-1}=o\left(n^{1 / 2}\right)$, then

$$
\left(\hat{\mathbf{H}}_{\tau}^{s p}\right)^{-1} \hat{\mathbf{\Omega}}_{\tau}^{s p}\left(\hat{\mathbf{H}}_{\tau}^{s p}\right)^{-1} \stackrel{p}{\rightarrow}\left(\mathbf{H}_{\tau}^{s p}\right)^{-1} \boldsymbol{\Omega}_{\tau}^{s p}\left(\mathbf{H}_{\tau}^{s p}\right)^{-1} .
$$

Proof of Theorem 4 can be found in Appendix A.2. 


\section{A.2 Proofs for the Main Theoretical Results}

Proof of Theorem 1. The proof relies on the result of Theorem 2.1 in Newey and McFadden (1994). To use this theorem, at first we need to show that

$$
E\left[F Z_{\tau}\left(q\left(\boldsymbol{\theta}_{1}\right), e\left(\boldsymbol{\theta}_{2}\right), Y\right) \mid T=c\right]
$$

is uniquely minimized at $\left(\boldsymbol{\theta}_{1, \tau}, \boldsymbol{\theta}_{2, \tau}\right)$. By Assumptions 2.2 and 2.5, the loss function $F Z_{\tau}(q, e, y)$ is strictly consistent for the $\tau$-quantile and conditional tail expectation of a random variable (Corollary 5.5 in Fissler and Ziegel (2016)). Thus $E\left[F Z_{\tau}\left(q\left(\boldsymbol{\theta}_{1}\right), e\left(\boldsymbol{\theta}_{2}\right), Y\right) \mid T=c\right]$ is uniquely minimized at $\left(Q_{Y \mid W, T=c}(\tau), C T E_{Y \mid W, T=c}(\tau)\right)$, which is specified by $\left(q\left(\boldsymbol{\theta}_{1, \tau}\right), e\left(\boldsymbol{\theta}_{2, \tau}\right)\right)$. By Assumption 2.6, it thus follows that $E\left[F Z_{\tau}\left(q\left(\boldsymbol{\theta}_{1}\right), e\left(\boldsymbol{\theta}_{2}\right), Y\right) \mid T=c\right]$ is uniquely minimized at $\left(\boldsymbol{\theta}_{1, \tau}, \boldsymbol{\theta}_{2, \tau}\right)$. Next if Assumption 1 holds, it can be shown that $\left(\boldsymbol{\theta}_{1, \tau}, \boldsymbol{\theta}_{2, \tau}\right)$ can also be obtained from

$$
\left(\boldsymbol{\theta}_{1, \tau}, \boldsymbol{\theta}_{2, \tau}\right)=\arg \min _{\left(\boldsymbol{\theta}_{1}, \boldsymbol{\theta}_{2}\right) \in \boldsymbol{\Theta}} E\left[\bar{K}\left(v_{0}, \boldsymbol{\gamma}_{0}\right) F Z_{\tau}\left(q\left(\boldsymbol{\theta}_{1}\right), e\left(\boldsymbol{\theta}_{2}\right), Y\right)\right]
$$

We then show that the following uniform convergence holds

$$
\begin{aligned}
\sup _{\left(\boldsymbol{\theta}_{1}, \boldsymbol{\theta}_{2}\right) \in \boldsymbol{\Theta}} & \mid \frac{1}{n} \sum_{i=1}^{n} \tilde{K}_{i}(\hat{v}, \hat{\gamma}) F Z_{\tau}\left(q_{i}\left(\boldsymbol{\theta}_{1}\right), e_{i}\left(\boldsymbol{\theta}_{2}\right), Y_{i}\right) \\
& -E\left[\bar{K}\left(v_{0}, \gamma_{0}\right) F Z_{\tau}\left(q\left(\boldsymbol{\theta}_{1}\right), e\left(\boldsymbol{\theta}_{2}\right), Y\right)\right] \mid \stackrel{p}{\rightarrow} 0
\end{aligned}
$$

By using the triangle inequality, we have

$$
\begin{array}{r}
\sup _{\left(\boldsymbol{\theta}_{1}, \boldsymbol{\theta}_{2}\right) \in \boldsymbol{\Theta}} \mid \frac{1}{n} \sum_{i=1}^{n} \tilde{K}_{i}(\hat{v}, \hat{\gamma}) F Z_{\tau}\left(q_{i}\left(\boldsymbol{\theta}_{1}\right), e_{i}\left(\boldsymbol{\theta}_{2}\right), Y_{i}\right) \\
-E\left[\bar{K}\left(v_{0}, \gamma_{0}\right) F Z_{\tau}\left(q\left(\boldsymbol{\theta}_{1}\right), e\left(\boldsymbol{\theta}_{2}\right), Y\right)\right] \mid \\
\leq \sup _{\left(\boldsymbol{\theta}_{1}, \boldsymbol{\theta}_{2}\right) \in \boldsymbol{\Theta}} \mid \frac{1}{n} \sum_{i=1}^{n} \tilde{K}_{i}(\hat{v}, \hat{\gamma}) F Z_{\tau}\left(q_{i}\left(\boldsymbol{\theta}_{1}\right), e_{i}\left(\boldsymbol{\theta}_{2}\right), Y_{i}\right) \\
-\frac{1}{n} \sum_{i=1}^{n} \bar{K}_{i}\left(v_{0}, \gamma_{0}\right) F Z_{\tau}\left(q_{i}\left(\boldsymbol{\theta}_{1}\right), e_{i}\left(\boldsymbol{\theta}_{2}\right), Y_{i}\right) \mid \\
+\sup _{\left(\boldsymbol{\theta}_{1}, \boldsymbol{\theta}_{2}\right) \in \boldsymbol{\Theta}} \mid \frac{1}{n} \sum_{i=1}^{n} \bar{K}_{i}\left(v_{0}, \gamma_{0}\right) F Z_{\tau}\left(q_{i}\left(\boldsymbol{\theta}_{1}\right), e_{i}\left(\boldsymbol{\theta}_{2}\right), Y_{i}\right) \\
-E\left[\bar{K}\left(v_{0}, \boldsymbol{\gamma}_{0}\right) F Z_{\tau}\left(q\left(\boldsymbol{\theta}_{1}\right), e\left(\boldsymbol{\theta}_{2}\right), Y\right)\right] \mid .
\end{array}
$$

For (33), it can be shown that it is $o_{p}(1)$, since

$$
\begin{aligned}
& \sup _{\left(\boldsymbol{\theta}_{1}, \boldsymbol{\theta}_{2}\right) \in \boldsymbol{\Theta}}\left|\frac{1}{n} \sum_{i=1}^{n}\left[\tilde{K}_{i}(\hat{v}, \hat{\gamma})-\bar{K}_{i}\left(v_{0}, \boldsymbol{\gamma}_{0}\right)\right] F Z_{\tau}\left(q_{i}\left(\boldsymbol{\theta}_{1}\right), e_{i}\left(\boldsymbol{\theta}_{2}\right), Y_{i}\right)\right| \\
& \leq \max _{i \in\{1, \ldots, n\}}\left|\tilde{K}_{i}(\hat{v}, \hat{\gamma})-\bar{K}_{i}\left(v_{0}, \gamma_{0}\right)\right| \times \sup _{\left(\boldsymbol{\theta}_{1}, \boldsymbol{\theta}_{2}\right) \in \boldsymbol{\Theta}} \frac{1}{n} \sum_{i=1}^{n}\left|F Z_{\tau}\left(q_{i}\left(\boldsymbol{\theta}_{1}\right), e_{i}\left(\boldsymbol{\theta}_{2}\right), Y_{i}\right)\right| \\
& \leq \max _{i \in\{1, \ldots, n\}}\left|\bar{K}_{i}(\hat{v}, \hat{\gamma})-\bar{K}_{i}\left(v_{0}, \gamma_{0}\right)\right| \times\left[\frac{1}{n} \sum_{i=1}^{n} b\left(V_{i}\right)\right] \\
& =o_{p}(1),
\end{aligned}
$$

where the second inequality above follows from Assumptions 2.7, 2.8 and Lipschitz continuity of the mapping $t \rightarrow \min \{\max \{t, 0\}, 1\}$ as well as the fact that $\bar{K}_{i}\left(v_{0}, \gamma_{0}\right) \in[0,1]$ under Assumption 1 (see Lemma 3.2 
of Abadie et al. (2002) so that $\bar{K}_{i}\left(v_{0}, \gamma_{0}\right)=\tilde{K}_{i}\left(v_{0}, \gamma_{0}\right)$ for each $i \in\{1, \ldots, n\}$.

Next we show that (34) is also $o_{p}(1)$. By Assumptions 2.4 and 2.5, $F Z_{\tau}\left(q\left(\boldsymbol{\theta}_{1}\right), e\left(\boldsymbol{\theta}_{2}\right), Y\right)$ is continuous in $\left(\boldsymbol{\theta}_{1}, \boldsymbol{\theta}_{2}\right) \in \boldsymbol{\Theta}$. In addition, by Assumption 2.7,

$$
E\left[\left|\bar{K}\left(v_{0}, \boldsymbol{\gamma}_{0}\right) F Z_{\tau}\left(q\left(\boldsymbol{\theta}_{1}\right), e\left(\boldsymbol{\theta}_{2}\right), Y\right)\right|\right] \leq E\left[\bar{K}\left(v_{0}, \boldsymbol{\gamma}\right) b(V)\right] \leq E[b(V)]<\infty
$$

Using these results along with Assumptions 2.1, 2.3 and 2.4, we can apply Lemma 2.4 of Newey and McFadden (1994) to deduce that (34) is also $o_{p}(1)$. Combining all of the above results, it then follows from Theorem 2.1 of Newey and McFadden (1994) that $\left(\hat{\boldsymbol{\theta}}_{1, \tau}, \hat{\boldsymbol{\theta}}_{2, \tau}\right) \stackrel{p}{\rightarrow}\left(\boldsymbol{\theta}_{1, \tau}, \boldsymbol{\theta}_{2, \tau}\right)$.

Proof of Lemma 1. Let $\mathcal{V}_{m}$ denote the support of the distribution of $\left(Y, W_{c}\right)$ conditional on $W_{d}=m$. We first show that, for each $m \in \mathcal{W}_{d}, \sup _{\left(y, w_{c}\right) \in \mathcal{V}_{m}}\left|\hat{v}_{m}\left(y, w_{c}\right)-v_{0, m}\left(y, w_{c}\right)\right|=o_{p}(1)$, which relies on using the result of Newey (1997) for the power series approximation. To use this result, in addition to Assumptions 2.1, 3.1 and 3.2, we also need to show that $\operatorname{Var}\left(Z \mid V_{m}\right)$ is bounded. This is evident since $Z$ is a binary variable. With these assumptions and above result, Theorem 4 of Newey (1997) implies that $\sup _{\left(y, w_{c}\right) \in \mathcal{V}_{m}}\left|\hat{v}_{m}-v_{0, m}\right|=o_{p}(1)$ under the conditions that $r+1<s_{m}, \kappa_{m} \rightarrow \infty$ and $\kappa_{m}^{3} / n \rightarrow 0$. By (26) and since $m$ takes a finite number of values, we can conclude that $\sup _{V \in \mathcal{V}}\left|\hat{v}-v_{0}\right|=o_{p}(1)$. Now consider $\bar{K}(\hat{v}, \hat{\gamma})$ as an estimator for $\bar{K}\left(v_{0}, \gamma_{0}\right)$. To verify Assumption 2.8, notice that

$$
\begin{aligned}
\sup _{V \in \mathcal{V}}\left|\bar{K}(V ; \hat{v}, \hat{\gamma})-\bar{K}\left(V ; v_{0}, \gamma_{0}\right)\right| \leq & \sup _{V \in \mathcal{V}}\left|\bar{K}(V ; \hat{v}, \hat{\gamma})-\bar{K}\left(V ; v_{0}, \hat{\gamma}\right)\right|+\sup _{V \in \mathcal{V}}\left|\bar{K}\left(V ; v_{0}, \hat{\gamma}\right)-\bar{K}\left(V ; v_{0}, \gamma_{0}\right)\right| \\
\leq & \sup _{V \in \mathcal{V}}\left|\frac{D\left(\hat{v}-v_{0}\right)}{1-\hat{\pi}}-\frac{(1-D)\left(\hat{v}-v_{0}\right)}{\hat{\pi}}\right| \\
& +\sup _{V \in \mathcal{V}}\left|D\left(1-v_{0}\right)\left(\frac{1}{1-\hat{\pi}}-\frac{1}{1-\pi_{0}}\right)\right| \\
& +\sup _{V \in \mathcal{V}}\left|(1-D) v_{0}\left(\frac{1}{\hat{\pi}}-\frac{1}{\pi_{0}}\right)\right| \\
\leq & \sup _{V \in \mathcal{V}}\left|\hat{v}-v_{0}\right| \sup _{V \in \mathcal{V}}\left|\frac{D}{1-\hat{\pi}}-\frac{(1-D)}{\hat{\pi}}\right| \\
& +\sup _{V \in \mathcal{V}}\left|D\left(1-v_{0}\right)\right| \sup _{V \in \mathcal{V}}\left\|\frac{1}{(1-\bar{\pi})^{2}} \nabla_{\gamma} \bar{\pi}\right\|\left\|\hat{\gamma}-\gamma_{0}\right\| \\
& +\sup _{V \in \mathcal{V}}\left|(1-D) v_{0}\right| \sup _{V \in \mathcal{V}}\left\|\frac{1}{\bar{\pi}^{2}} \nabla_{\gamma} \bar{\pi}\right\|\left\|\hat{\gamma}-\gamma_{0}\right\|,
\end{aligned}
$$

where $\hat{\pi}:=\pi(X, \hat{\gamma}), \pi_{0}:=\pi\left(X, \gamma_{0}\right), \bar{\pi}:=\pi(X, \bar{\gamma})$, and $\nabla_{\gamma} \bar{\pi}$ is the partial derivative $\nabla_{\gamma} \pi$ evaluated at $\gamma=\bar{\gamma}$, where $\bar{\gamma}$ is some point between $\gamma_{0}$ and $\hat{\gamma}$. Since $\sup _{V \in \mathcal{V}}\left|\hat{v}-v_{0}\right|=o_{p}(1)$ and $\pi(x, \gamma)$ is bounded away from 0 and 1 over the support of $X$ and for every $\gamma$ such that $\left\|\boldsymbol{\gamma}-\gamma_{0}\right\| \leq \varepsilon$, the term (35) is thus $o_{p}(1)$. Since $v_{0}=E[Z \mid V]$ is bounded for $V \in \mathcal{V}$ and $D \in\{1,0\}$, the terms (36) and (37) are also $o_{p}(1)$ under Assumptions 3.3 and 3.4. Combining the above results, we can conclude that $\sup _{V \in \mathcal{V}}\left|\bar{K}(V ; \hat{v}, \hat{\gamma})-\bar{K}\left(V ; v_{0}, \gamma_{0}\right)\right|=o_{p}(1)$.

Proof of Theorem 2. The proof relies on using the result of Theorem 5.23 in van der Vaart (1998). First, we note that measurability (w.r.t. $(Y, W)$ ) and almost surely differentiability (w.r.t. $\boldsymbol{\theta}_{1}$ and $\boldsymbol{\theta}_{2}$ ) of $\bar{K}(\hat{v}, \hat{\gamma}) F Z_{\tau}\left(q\left(\boldsymbol{\theta}_{1}\right), e_{1}\left(\boldsymbol{\theta}_{2}\right), Y\right)$ can be proved by using the fact that $F Z_{\tau}\left(q\left(\boldsymbol{\theta}_{1}\right), e_{1}\left(\boldsymbol{\theta}_{2}\right), Y\right)$ has these properties (Dimitriadis and Bayer, 2019) and $\bar{K}(\hat{v}, \hat{\gamma})$ is also a measurable function of $(Y, W)$. In addition, it can be shown that $F Z_{\tau}\left(q\left(\boldsymbol{\theta}_{1}\right), e_{1}\left(\boldsymbol{\theta}_{2}\right), Y\right)$ is locally Lipschitz continuous in $\left(\boldsymbol{\theta}_{1}, \boldsymbol{\theta}_{2}\right)$ around $\left(\boldsymbol{\theta}_{1, \tau}, \boldsymbol{\theta}_{2, \tau}\right)$ (see Lemma 2 in Appendix A.3). By Assumption 4.2 and using Lemma 2 in Appendix A.3, there exists a 
constant $A_{\tau}^{*}(V)$ such that

$$
\begin{aligned}
E\left[\left|\bar{K}\left(v_{0}, \gamma_{0}\right)+o_{p}(1)\right|^{2}\left(A_{\tau}^{*}(V)\right)^{2}\right] & \cong E\left[\left(\bar{K}\left(v_{0}, \gamma_{0}\right)\right)^{2}\left(A_{\tau}^{*}(V)\right)^{2}\right] \\
& \leq E\left[\bar{K}\left(v_{0}, \gamma_{0}\right)\left(A_{\tau}^{*}(V)\right)^{2}\right] \\
& =E\left[\left(A_{\tau}^{*}(V)\right)^{2} \mid T=c\right] P(T=c) \\
& <\infty .
\end{aligned}
$$

We now show that $E\left[\bar{K}\left(v_{0}, \boldsymbol{\gamma}_{0}\right) F Z_{\tau}\left(q\left(\boldsymbol{\theta}_{1}\right), e\left(\boldsymbol{\theta}_{2}\right), Y\right)\right]$ admits a second order Taylor expansion at $\left(\boldsymbol{\theta}_{1, \tau}, \boldsymbol{\theta}_{2, \tau}\right)$. Under Assumptions 2.4 and 2.5, $E\left[\bar{K}\left(v_{0}, \gamma_{0}\right) F Z_{\tau}\left(q\left(\boldsymbol{\theta}_{1}\right), e\left(\boldsymbol{\theta}_{2}\right), Y\right)\right]$ is a twice differentiable function of $\boldsymbol{\theta}:=\left(\boldsymbol{\theta}_{1}, \boldsymbol{\theta}_{2}\right)$ at $\left(\boldsymbol{\theta}_{1, \tau}, \boldsymbol{\theta}_{2, \tau}\right)$, and it can be shown that

$$
\begin{aligned}
\mathbf{H}_{\tau} & :=\nabla_{\boldsymbol{\theta} \boldsymbol{\theta}} E\left[\bar{K}\left(v_{0}, \boldsymbol{\gamma}_{0}\right) F Z_{\tau}\left(q\left(\boldsymbol{\theta}_{1, \tau}\right), e\left(\boldsymbol{\theta}_{2, \tau}\right), Y\right)\right] \\
& =\nabla_{\boldsymbol{\theta} \boldsymbol{\theta}} E\left[F Z_{\tau}\left(q\left(\boldsymbol{\theta}_{2, \tau}\right), e\left(\boldsymbol{\theta}_{2, \tau}\right), Y\right) \mid T=c\right] \times P(T=c),
\end{aligned}
$$

where

$$
\nabla_{\boldsymbol{\theta} \boldsymbol{\theta}} E\left[F Z_{\tau}\left(q\left(\boldsymbol{\theta}_{1, \tau}\right), e\left(\boldsymbol{\theta}_{2, \tau}\right), Y\right) \mid T=c\right]=\left(\begin{array}{cc}
\mathbf{C}_{\tau, 11} & \mathbf{0} \\
\mathbf{0} & \mathbf{C}_{\tau, 22}
\end{array}\right)
$$

and

$$
\begin{aligned}
\mathbf{C}_{\tau, 11}= & E\left[f_{Y \mid W, T=c}\left(q\left(\boldsymbol{\theta}_{1, \tau}\right)\right)\left[G_{1}^{\prime}\left(q\left(\boldsymbol{\theta}_{1, \tau}\right)\right)+\frac{1}{\tau} G_{2}^{\prime}\left(e\left(\boldsymbol{\theta}_{2, \tau}\right)\right)\right]\right. \\
& \left.\times\left[\nabla_{\boldsymbol{\theta}_{1}} q\left(\boldsymbol{\theta}_{1, \tau}\right)\right]^{\top}\left[\nabla_{\boldsymbol{\theta}_{1}} q\left(\boldsymbol{\theta}_{1, \tau}\right)\right] \mid T=c\right], \\
\mathbf{C}_{\tau, 22}= & E\left[G_{2}^{\prime \prime}\left(e\left(\boldsymbol{\theta}_{2, \tau}\right)\right)\left[\nabla_{\boldsymbol{\theta}_{2}} e\left(\boldsymbol{\theta}_{2, \tau}\right)\right]^{\top}\left[\nabla_{\boldsymbol{\theta}_{2}} e\left(\boldsymbol{\theta}_{2, \tau}\right)\right] \mid T=c\right] .
\end{aligned}
$$

With the above results, using Theorem 5.23 in van der Vaart (1998), we can have

$$
\begin{aligned}
\sqrt{n}\left(\left(\hat{\boldsymbol{\theta}}_{1, \tau}, \hat{\boldsymbol{\theta}}_{2, \tau}\right)-\left(\boldsymbol{\theta}_{1, \tau}, \boldsymbol{\theta}_{2, \tau}\right)\right)= & -\mathbf{H}_{\tau}^{-1} \frac{1}{\sqrt{n}} \sum_{i=1}^{n} \bar{K}_{i}(\hat{v}, \hat{\gamma}) \nabla_{\boldsymbol{\theta}} F Z_{\tau}\left(q_{i}\left(\boldsymbol{\theta}_{1, \tau}\right), e_{i}\left(\boldsymbol{\theta}_{2, \tau}\right), Y_{i}\right)+o_{p}(1) \\
= & -\mathbf{H}_{\tau}^{-1}\left\{\frac{1}{\sqrt{n}} \sum_{i=1}^{n} \bar{K}_{i}\left(\hat{v}, \boldsymbol{\gamma}_{0}\right) \nabla_{\boldsymbol{\theta}} F Z_{\tau}\left(q_{i}\left(\boldsymbol{\theta}_{1, \tau}\right), e_{i}\left(\boldsymbol{\theta}_{2, \tau}\right), Y_{i}\right)\right. \\
& \left.+\frac{1}{n} \sum_{i=1}^{n}\left[\nabla_{\boldsymbol{\gamma}} \bar{K}_{i}\left(\hat{v}, \boldsymbol{\gamma}_{0}\right)\right]^{\top} \sqrt{n}\left(\hat{\gamma}-\boldsymbol{\gamma}_{0}\right) \nabla_{\boldsymbol{\theta}} F Z_{\tau}\left(q_{i}\left(\boldsymbol{\theta}_{1, \tau}\right), e_{i}\left(\boldsymbol{\theta}_{2, \tau}\right), Y_{i}\right)\right\} \\
& +o_{p}(1),
\end{aligned}
$$

where

$$
\nabla_{\gamma} \bar{K}_{i}\left(\hat{v}, \gamma_{0}\right)=\left[\frac{\left(1-D_{i}\right) \hat{v}_{i}}{\pi_{0 i}^{2}}-\frac{D_{i}\left(1-\hat{v}_{i}\right)}{\left(1-\pi_{0 i}\right)^{2}}\right] \nabla_{\gamma} \bar{\pi}_{i},
$$

and $\bar{\pi}_{i}=\pi\left(X_{i}, \bar{\gamma}\right)$ and $\bar{\gamma}$ is some point between $\hat{\gamma}$ and $\gamma_{0}$, and

$$
\nabla_{\boldsymbol{\theta}} F Z_{\tau}\left(q_{i}\left(\boldsymbol{\theta}_{1, \tau}\right), e_{i}\left(\boldsymbol{\theta}_{2, \tau}\right), Y_{i}\right)=\left(\begin{array}{c}
\left(1\left\{Y_{i} \leq q\left(\boldsymbol{\theta}_{1, \tau}\right)\right\}-\tau\right) \\
\times\left[G_{1}^{\prime}\left(q_{i}\left(\boldsymbol{\theta}_{1, \tau}\right)\right)+\frac{1}{\tau} G_{2}^{\prime}\left(e_{i}\left(\boldsymbol{\theta}_{2, \tau}\right)\right)\right] \nabla_{\boldsymbol{\theta}_{1}} q_{i}\left(\boldsymbol{\theta}_{1, \tau}\right) \\
G_{2}^{\prime \prime}\left(e_{i}\left(\boldsymbol{\theta}_{2, \tau}\right)\right)\left[e_{i}\left(\boldsymbol{\theta}_{2, \tau}\right)\right. \\
\left.+\frac{1}{\tau} \max \left(q_{i}\left(\boldsymbol{\theta}_{1, \tau}\right)-Y_{i}, 0\right)-q_{i}\left(\boldsymbol{\theta}_{1, \tau}\right)\right] \nabla_{\boldsymbol{\theta}_{2}} e_{i}\left(\boldsymbol{\theta}_{2, \tau}\right)
\end{array}\right)
$$

is the Jacobian vector. By similar arguments used in proving Lemma A.1 in Abadie et al. (2002), with 
Assumption 4.5, it can be shown that

$$
\begin{aligned}
\frac{1}{\sqrt{n}} \sum_{i=1}^{n} \bar{K}_{i}\left(\hat{v}, \boldsymbol{\gamma}_{0}\right) \nabla_{\boldsymbol{\theta}} F Z_{\tau}\left(q_{i}\left(\boldsymbol{\theta}_{1, \tau}\right), e_{i}\left(\boldsymbol{\theta}_{2, \tau}\right), Y_{i}\right)= & \frac{1}{\sqrt{n}} \sum_{i=1}^{n} K_{i}\left(\boldsymbol{\gamma}_{0}\right) \nabla_{\boldsymbol{\theta}} F Z_{\tau}\left(q_{i}\left(\boldsymbol{\theta}_{1, \tau}\right), e_{i}\left(\boldsymbol{\theta}_{2, \tau}\right), Y_{i}\right) \\
& +o_{p}(1), \\
\frac{1}{n} \sum_{i=1}^{n}\left[\nabla_{\boldsymbol{\gamma}} \bar{K}_{i}\left(\hat{v}, \boldsymbol{\gamma}_{0}\right)\right]^{\top} \sqrt{n}\left(\hat{\gamma}-\boldsymbol{\gamma}_{0}\right) \nabla_{\boldsymbol{\theta}} F Z_{\tau}\left(q_{i}\left(\boldsymbol{\theta}_{1, \tau}\right), e_{i}\left(\boldsymbol{\theta}_{2, \tau}\right), Y_{i}\right)= & \frac{1}{n} \sum_{i=1}^{n}\left[\nabla_{\gamma} K_{i}\left(\boldsymbol{\gamma}_{0}\right)\right]^{\top} \sqrt{n}\left(\hat{\gamma}-\gamma_{0}\right) \\
& \times \nabla_{\boldsymbol{\theta}} F Z_{\tau}\left(q_{i}\left(\boldsymbol{\theta}_{1, \tau}\right), e_{i}\left(\boldsymbol{\theta}_{2, \tau}\right), Y_{i}\right)+o_{p}(1),
\end{aligned}
$$

where

$$
\begin{aligned}
K_{i}\left(\gamma_{0}\right) & =1-\frac{D_{i}\left(1-Z_{i}\right)}{1-\pi_{0 i}}-\frac{\left(1-D_{i}\right) Z_{i}}{\pi_{0 i}} \\
\nabla_{\gamma} K_{i}\left(\gamma_{0}\right) & =\left[\frac{\left(1-D_{i}\right) Z_{i}}{\pi_{0 i}^{2}}-\frac{D_{i}\left(1-Z_{i}\right)}{\left(1-\pi_{0 i}\right)^{2}}\right] \nabla_{\gamma} \bar{\pi}_{i} .
\end{aligned}
$$

It further can be shown that

$$
\frac{1}{n} \sum_{i=1}^{n}\left[\nabla_{\boldsymbol{\gamma}} K_{i}\left(\boldsymbol{\gamma}_{0}\right)\right]^{\top} \sqrt{n}\left(\hat{\gamma}-\gamma_{0}\right) \nabla_{\boldsymbol{\theta}} F Z_{\tau}\left(q_{i}\left(\boldsymbol{\theta}_{1, \tau}\right), e_{i}\left(\boldsymbol{\theta}_{2, \tau}\right), Y_{i}\right) \stackrel{p}{\rightarrow} \frac{1}{\sqrt{n}} \sum_{i=1}^{n} \mathbf{M}_{\tau} \psi\left(X_{i}\right),
$$

where

$$
\mathbf{M}_{\tau}=E\left[\nabla_{\boldsymbol{\theta}} F Z_{\tau}\left(q_{i}\left(\boldsymbol{\theta}_{1, \tau}\right), e_{i}\left(\boldsymbol{\theta}_{2, \tau}\right), Y_{i}\right)\left[\nabla_{\boldsymbol{\gamma}} K_{i}\left(\boldsymbol{\gamma}_{0}\right)\right]^{\top}\right] .
$$

Therefore we can conclude that

$$
\begin{aligned}
\sqrt{n}\left(\left(\hat{\boldsymbol{\theta}}_{1, \tau}, \hat{\boldsymbol{\theta}}_{2, \tau}\right)-\left(\boldsymbol{\theta}_{1, \tau}, \boldsymbol{\theta}_{2, \tau}\right)\right)= & -\mathbf{H}_{\tau}^{-1}\left\{\frac{1}{\sqrt{n}} \sum_{i=1}^{n} K_{i}\left(\boldsymbol{\gamma}_{0}\right) \nabla_{\boldsymbol{\theta}} F Z_{\tau}\left(q_{i}\left(\boldsymbol{\theta}_{1, \tau}\right), e_{i}\left(\boldsymbol{\theta}_{2, \tau}\right), Y_{i}\right)+\right. \\
& \left.+\frac{1}{\sqrt{n}} \sum_{i=1}^{n} \mathbf{M}_{\tau} \psi\left(X_{i}\right)\right\}+o_{p}(1),
\end{aligned}
$$

and

$$
\sqrt{n}\left(\left(\hat{\boldsymbol{\theta}}_{1, \tau}, \hat{\boldsymbol{\theta}}_{2, \tau}\right)-\left(\boldsymbol{\theta}_{1, \tau}, \boldsymbol{\theta}_{2, \tau}\right)\right) \stackrel{d}{\rightarrow} N\left(\mathbf{0}, \mathbf{H}_{\tau}^{-1} \boldsymbol{\Omega}_{\tau} \mathbf{H}_{\tau}^{-1}\right)
$$

where $\boldsymbol{\Omega}_{\tau}=E\left[\mathbf{J}_{\tau} \mathbf{J}_{\tau}^{\top}\right]$ and

$$
\mathbf{J}_{\tau}=K\left(\gamma_{0}\right) \nabla_{\boldsymbol{\theta}} F Z_{\tau}\left(q\left(\boldsymbol{\theta}_{1, \tau}\right), e\left(\boldsymbol{\theta}_{2, \tau}\right), Y\right)+\mathbf{M}_{\tau} \psi(X) .
$$

Proof of Theorem 3. At first, we show that the empirical loss function converges to the true loss function uniformly over $\mathcal{T} \times \boldsymbol{\Theta}$, which is a compact metric space. We use results of Newey (1991), which requires Assumption 5.1, and the following conditions: (i) pointwise convergence between the empirical loss function and the true loss function for each $(\tau, \boldsymbol{\theta}) \in \mathcal{T} \times \boldsymbol{\Theta}$, (ii) $n^{-1} \sum_{i=1}^{n} \tilde{K}_{i}(\hat{\nu}, \hat{\gamma}) h_{i}(\tau, \boldsymbol{\theta})$ is stochastic equicontinuous on $(\tau, \boldsymbol{\theta})$, and (iii) $E\left[\bar{K}\left(v_{0}, \gamma_{0}\right) h(\tau, \boldsymbol{\theta})\right]$ is equicontinuous.

For (i), we have shown in Theorem 1 that if Assumptions 2.1, 2.2 and 2.4-2.8 in Theorem 1 and Assumption 5.1 (replaces Assumption 2.3) hold, for each $\tau \in \mathcal{T}$,

$$
\sup _{\boldsymbol{\theta} \in \boldsymbol{\Theta}}\left|\frac{1}{n} \sum_{i=1}^{n} \tilde{K}_{i}(\hat{\nu}, \hat{\gamma}) h_{i}(\tau, \boldsymbol{\theta})-E\left[\bar{K}\left(v_{0}, \boldsymbol{\gamma}_{0}\right) h(\tau, \boldsymbol{\theta})\right]\right|=o_{p}(1) .
$$


Thus for each $(\tau, \boldsymbol{\theta}) \in \mathcal{T} \times \boldsymbol{\Theta}$, the pointwise convergence of $(38)$ also holds. For (ii), at first let $\boldsymbol{\theta}^{1}:=\left(\boldsymbol{\theta}_{1}^{1}, \boldsymbol{\theta}_{2}^{1}\right)$ and $\boldsymbol{\theta}^{2}:=\left(\boldsymbol{\theta}_{1}^{2}, \boldsymbol{\theta}_{2}^{2}\right)$ be two vectors of parameter values. Then with Assumptions 5.1 and 2.4, using the result of Lemma 3 in Appendix A.4, we can have

$$
\begin{array}{r}
\left|\frac{1}{n} \sum_{i=1}^{n} \tilde{K}_{i}(\hat{\nu}, \hat{\gamma}) h_{i}\left(\tau_{1}, \boldsymbol{\theta}^{1}\right)-\frac{1}{n} \sum_{i=1}^{n} \tilde{K}_{i}(\hat{\nu}, \hat{\gamma}) h_{i}\left(\tau_{2}, \boldsymbol{\theta}^{2}\right)\right| \\
\leq \frac{1}{n} \sum_{i=1}^{n}\left|\left(\bar{K}_{i}\left(v_{0}, \boldsymbol{\gamma}_{0}\right)+o_{p}(1)\right)\left(h_{i}\left(\tau_{1}, \boldsymbol{\theta}^{1}\right)-h_{i}\left(\tau_{2}, \boldsymbol{\theta}^{2}\right)\right)\right| \\
\leq \frac{1}{n} \sum_{i=1}^{n}\left|\bar{K}_{i}\left(v_{0}, \gamma_{0}\right)+o_{p}(1)\right|\left|h_{i}\left(\tau_{1}, \boldsymbol{\theta}^{1}\right)-h_{i}\left(\tau_{2}, \boldsymbol{\theta}^{2}\right)\right| \\
\leq\left(\left\|\tau_{1}-\tau_{2}\right\|+\left\|\boldsymbol{\theta}^{1}-\boldsymbol{\theta}^{2}\right\|\right)\left[\frac{1}{n} \sum_{i=1}^{n}\left|\bar{K}_{i}\left(v_{0}, \boldsymbol{\gamma}_{0}\right)+o_{p}(1)\right| B^{*}\left(V_{i}\right)\right],
\end{array}
$$

where $B^{*}\left(V_{i}\right)$ is the constant defined in Lemma 3. Notice that $\left|\bar{K}_{i}\left(v_{0}, \gamma_{0}\right)+o_{p}(1)\right| B^{*}\left(V_{i}\right) \geq 0$ for every $i=1, \ldots, n$, and $B^{*}\left(V_{i}\right)$ is determined by a sum of $\ln 2$ and constants $B_{k}(V), k=1, \ldots, 5$. With Assumption 5.3, it can be shown that

$$
\begin{aligned}
\frac{1}{n} \sum_{i=1}^{n}\left|\bar{K}_{i}\left(v_{0}, \gamma_{0}\right)+o_{p}(1)\right| B^{*}\left(V_{i}\right) & \leq \frac{1}{n} \sum_{i=1}^{n}\left(\left|\bar{K}_{i}\left(v_{0}, \gamma_{0}\right)\right|+\left|o_{p}(1)\right|\right) B^{*}\left(V_{i}\right) \\
& =\frac{1}{n} \sum_{i=1}^{n}\left|\bar{K}_{i}\left(v_{0}, \gamma_{0}\right)\right| B^{*}\left(V_{i}\right)+\left(\frac{1}{n} \sum_{i=1}^{n}\left|o_{p}(1)\right| B^{*}\left(V_{i}\right)\right) \\
& \stackrel{p}{\rightarrow} E\left[B^{*}\left(V_{i}\right) \mid T=c\right] P(T=c) \\
& <\infty .
\end{aligned}
$$

Therefore we can conclude that $n^{-1} \sum_{i=1}^{n}\left|\bar{K}_{i}\left(v_{0}, \gamma_{0}\right)+o_{p}(1)\right| B^{*}\left(V_{i}\right)$ is $O_{p}(1)$ and the empirical loss $n^{-1} \sum_{i=1}^{n} \tilde{K}_{i}(\hat{\nu}, \hat{\gamma}) h_{i}(\tau, \boldsymbol{\theta})$ is stochastic equicontinuous on $(\tau, \boldsymbol{\theta}) \in \mathcal{T} \times \boldsymbol{\Theta}$. Finally, for (iii), with Assumption $5.2, E\left[\bar{K}\left(v_{0}, \gamma_{0}\right) h(\tau, \boldsymbol{\theta})\right]$ is equicontinuous since its first order derivatives w.r.t. $(\tau, \boldsymbol{\theta}) \in \mathcal{T} \times \boldsymbol{\Theta}$ is bound. Combining the above results, with Corollary 2.1 of Newey (1991), we can conclude that

$$
\sup _{(\tau, \boldsymbol{\theta}) \in \mathcal{T} \times \boldsymbol{\Theta}}\left|\frac{1}{n} \sum_{i=1}^{n} \tilde{K}_{i}(\hat{\nu}, \hat{\gamma}) h_{i}(\tau, \boldsymbol{\theta})-E\left[\bar{K}\left(v_{0}, \boldsymbol{\gamma}_{0}\right) h(\tau, \boldsymbol{\theta})\right]\right|=o_{p}(1) .
$$

Then uniformly over $\tau \in \mathcal{T}$, for any $\varepsilon>0$, with probability approaching one, we have

$$
\begin{aligned}
\frac{1}{n} \sum_{i=1}^{n} \tilde{K}_{i}(\hat{\nu}, \hat{\gamma}) h_{i}\left(\tau, \hat{\boldsymbol{\theta}}_{\tau}\right) & <\frac{1}{n} \sum_{i=1}^{n} \tilde{K}_{i}(\hat{\nu}, \hat{\gamma}) h_{i}\left(\tau, \boldsymbol{\theta}_{\tau}\right)+\frac{\varepsilon}{3}, \\
E\left[\bar{K}\left(v_{0}, \boldsymbol{\gamma}_{0}\right) h\left(\tau, \hat{\boldsymbol{\theta}}_{\tau}\right)\right] & <\frac{1}{n} \sum_{i=1}^{n} \tilde{K}_{i}(\hat{\nu}, \hat{\gamma}) h_{i}\left(\tau, \hat{\boldsymbol{\theta}}_{\tau}\right)+\frac{\varepsilon}{3}, \\
\frac{1}{n} \sum_{i=1}^{n} \tilde{K}_{i}(\hat{\nu}, \hat{\gamma}) h_{i}\left(\tau, \boldsymbol{\theta}_{\tau}\right) & <E\left[\bar{K}\left(v_{0}, \boldsymbol{\gamma}_{0}\right) h\left(\tau, \boldsymbol{\theta}_{\tau}\right)\right]+\frac{\varepsilon}{3} .
\end{aligned}
$$

The inequality 39 holds because $\hat{\boldsymbol{\theta}}_{\tau}$ minimizes $n^{-1} \sum_{i=1}^{n} \bar{K}_{i}(\hat{\nu}, \hat{\gamma}) h_{i}(\tau, \boldsymbol{\theta})$ and the inequalities 40 and (41) hold because of (38). With (39), (40) and (41), we can conclude that

$$
E\left[\bar{K}\left(v_{0}, \gamma_{0}\right) h\left(\tau, \hat{\boldsymbol{\theta}}_{\tau}\right)\right]<E\left[\bar{K}\left(v_{0}, \boldsymbol{\gamma}_{0}\right) h\left(\tau, \boldsymbol{\theta}_{\tau}\right)\right]+\varepsilon
$$

holds uniformly over $\tau \in \mathcal{T}$ with probability approaching one. Let $\mathcal{M}$ be any open set in $\Theta$ containing 
$\boldsymbol{\theta}_{\tau}:=\left(\boldsymbol{\theta}_{1, \tau}, \boldsymbol{\theta}_{2, \tau}\right)$. Notice that $\mathcal{M}^{c} \cap \boldsymbol{\Theta}$ is compact. Also $E\left[\bar{K}\left(v_{0}, \gamma_{0}\right) h(\tau, \boldsymbol{\theta})\right]$ is continuous in $\boldsymbol{\theta}$ by Assumption 2.4 and is uniquely minimized at $\boldsymbol{\theta}_{\tau}$ by Assumptions 2.5 and 2.6. Therefore

$$
\inf _{\boldsymbol{\theta} \in \mathcal{M}^{c} \cap \boldsymbol{\Theta}} E\left[\bar{K}\left(v_{0}, \gamma_{0}\right) h(\tau, \boldsymbol{\theta})\right]=E\left[\bar{K}\left(v_{0}, \boldsymbol{\gamma}_{0}\right) h\left(\tau, \boldsymbol{\theta}^{*}\right)\right]>E\left[\bar{K}\left(v_{0}, \boldsymbol{\gamma}_{0}\right) h\left(\tau, \boldsymbol{\theta}_{\tau}\right)\right]
$$

for some $\boldsymbol{\theta}^{*} \in \mathcal{M}^{c} \cap \boldsymbol{\Theta}$. Then setting

$$
\varepsilon=\inf _{\boldsymbol{\theta} \in \mathcal{M}^{c} \cap \Theta} E\left[\bar{K}\left(v_{0}, \boldsymbol{\gamma}_{0}\right) h(\tau, \boldsymbol{\theta})\right]-E\left[\bar{K}\left(v_{0}, \boldsymbol{\gamma}_{0}\right) h\left(\tau, \boldsymbol{\theta}_{\tau}\right)\right]
$$

we can have

$$
E\left[\bar{K}\left(v_{0}, \boldsymbol{\gamma}_{0}\right) h\left(\tau, \hat{\boldsymbol{\theta}}_{\tau}\right)\right]<\inf _{\boldsymbol{\theta} \in \mathcal{M}^{c} \cap \Theta} E\left[\bar{K}\left(v_{0}, \boldsymbol{\gamma}_{0}\right) h(\tau, \boldsymbol{\theta})\right]
$$

holds uniformly over $\tau \in \mathcal{T}$ with probability approaching one. Thus $\hat{\boldsymbol{\theta}}_{\tau} \in \mathcal{M}$ uniformly over $\tau \in \mathcal{T}$ with probability approaching one.

Proof of Theorem 4. It is straightforward to show that $n^{-1} \sum_{i=1}^{n} \hat{\mathbf{J}}_{i, \tau}^{s p} \hat{\mathbf{J}}_{i, \tau}^{s p \top} \stackrel{p}{\rightarrow} \boldsymbol{\Omega}_{\tau}^{s p}$ when the assumptions hold. We thus focus on proving $\hat{\mathbf{H}}_{\tau}^{s p} \stackrel{p}{\rightarrow} \mathbf{H}_{\tau}^{s p}$. We show that $\hat{\mathbf{C}}_{\tau, 11}^{s p *} \stackrel{p}{\rightarrow} \mathbf{C}_{\tau, 11}^{s p *}$ and similar arguments can be used to prove $\hat{\mathbf{C}}_{\tau, 22}^{s p *} \stackrel{p}{\rightarrow} \mathbf{C}_{\tau, 22}^{s p *}$. To see this, notice that

$$
\left\|\hat{\mathbf{C}}_{\tau, 11}^{s p *}-\mathbf{C}_{\tau, 11}^{s p *}\right\| \leq I_{1}+I_{2}+I_{3}+I_{4}+I_{5}
$$

where

$$
\begin{aligned}
I_{1}= & \left\|\hat{\mathbf{C}}_{\tau, 11}^{s p *}-\frac{1}{n} \sum_{i=1}^{n}\left\{\bar{K}_{i}\left(v_{0}, \gamma_{0}\right) \varsigma_{\lambda, i}\left(\hat{\boldsymbol{\theta}}_{1, \tau}\right) \frac{1}{\tau} G_{s p}^{\prime}\left(e\left(\hat{\boldsymbol{\theta}}_{2, \tau}\right)\right) W_{i} W_{i}^{\top}\right\}\right\|, \\
I_{2}= & \| \frac{1}{n} \sum_{i=1}^{n}\left\{\bar{K}_{i}\left(v_{0}, \gamma_{0}\right) \varsigma_{\lambda_{n}, i}\left(\hat{\boldsymbol{\theta}}_{1, \tau}\right) \frac{1}{\tau} G_{s p}^{\prime}\left(e_{i}\left(\hat{\boldsymbol{\theta}}_{2, \tau}\right)\right) W_{i} W_{i}^{\top}\right\} \\
& -\frac{1}{n} \sum_{i=1}^{n}\left\{\bar{K}_{i}\left(v_{0}, \gamma_{0}\right) \varsigma_{\lambda_{n}, i}\left(\boldsymbol{\theta}_{1, \tau}\right) \frac{1}{\tau} G_{s p}^{\prime}\left(e_{i}\left(\hat{\boldsymbol{\theta}}_{2, \tau}\right)\right) W_{i} W_{i}^{\top}\right\} \|, \\
I_{3}= & \| \frac{1}{n} \sum_{i=1}^{n}\left\{\bar{K}_{i}\left(v_{0}, \gamma_{0}\right) \varsigma_{\lambda_{n}, i}\left(\boldsymbol{\theta}_{1, \tau}\right) \frac{1}{\tau} G_{s p}^{\prime}\left(e_{i}\left(\hat{\boldsymbol{\theta}}_{2, \tau}\right)\right) W_{i} W_{i}^{\top}\right\} \\
& -\frac{1}{n} \sum_{i=1}^{n}\left\{\bar{K}_{i}\left(v_{0}, \gamma_{0}\right) \varsigma_{\lambda_{n}, i}\left(\boldsymbol{\theta}_{1, \tau}\right) \frac{1}{\tau} G_{s p}^{\prime}\left(e_{i}\left(\boldsymbol{\theta}_{2, \tau}\right)\right) W_{i} W_{i}^{\top}\right\} \|, \\
I_{4}= & \| \frac{1}{n} \sum_{i=1}^{n}\left\{\bar{K}_{i}\left(v_{0}, \gamma_{0}\right) \varsigma_{\lambda_{n}, i}\left(\boldsymbol{\theta}_{1, \tau}\right) \frac{1}{\tau} G_{s p}^{\prime}\left(e_{i}\left(\boldsymbol{\theta}_{2, \tau}\right)\right) W_{i} W_{i}^{\top}\right\} \\
& -E\left[\bar{K}\left(v_{0}, \gamma_{0}\right) \varsigma_{\lambda_{n}}\left(\boldsymbol{\theta}_{1, \tau}\right) \frac{1}{\tau} G_{s p}^{\prime}\left(e\left(\boldsymbol{\theta}_{2, \tau}\right)\right) W W^{\top}\right] \|, \\
I_{5}= & \left\|E\left[\bar{K}\left(v_{0}, \gamma_{0}\right) \varsigma_{\lambda_{n}}\left(\boldsymbol{\theta}_{1, \tau}\right) \frac{1}{\tau} G_{s p}^{\prime}\left(e\left(\boldsymbol{\theta}_{2, \tau}\right)\right) W W^{\top}\right]-\mathbf{C}_{\tau, 11}^{s p *}\right\| .
\end{aligned}
$$


For $I_{1}$, it can be shown that

$$
\begin{aligned}
I_{1} \leq & \sup \left|\tilde{K}_{i}(\hat{v}, \hat{\gamma})-\bar{K}_{i}\left(v_{0}, \gamma_{0}\right)\right| \\
& \times \frac{1}{n} \sum_{i=1}^{n}\left|\varsigma_{\lambda, i}\left(\hat{\boldsymbol{\theta}}_{1, \tau}\right) \frac{1}{\tau} G_{s p}^{\prime}\left(e\left(\hat{\boldsymbol{\theta}}_{2, \tau}\right)\right) \times W_{i} W_{i}^{\top}\right| \\
\leq & \sup \left|\bar{K}_{i}(\hat{v}, \hat{\gamma})-\bar{K}_{i}\left(v_{0}, \gamma_{0}\right)\right| \times \\
& \frac{1}{n} \sum_{i=1}^{n}\left|\varsigma_{\lambda, i}\left(\hat{\boldsymbol{\theta}}_{1, \tau}\right) \frac{1}{\tau} G_{s p}^{\prime}\left(e\left(\hat{\boldsymbol{\theta}}_{2, \tau}\right)\right) \times W_{i} W_{i}^{\top}\right| \\
= & o_{p}(1)
\end{aligned}
$$

Next, convergence between $\varsigma_{\lambda_{n}, i}\left(\hat{\boldsymbol{\theta}}_{1, \tau}\right)$ and $\varsigma_{\lambda_{n}, i}\left(\boldsymbol{\theta}_{1, \tau}\right)$ can be constructed by using the fact that

$$
\frac{1}{\lambda_{n}}\left\|\hat{\boldsymbol{\theta}}_{1, \tau}-\boldsymbol{\theta}_{1, \tau}\right\|=o_{p}(1)
$$

and similar arguments in Powell (1984) and Engle and Manganelli (2004). Together with the facts that $\frac{1}{\tau} G_{s p}^{\prime}\left(e\left(\hat{\boldsymbol{\theta}}_{2, \tau}\right)\right), \varsigma_{\lambda_{n}, i}($.$) and E\left[\left\|W W^{\top}\right\| \mid T=c\right]$ are bounded and $G_{s p}^{\prime}\left(e_{i}\left(\boldsymbol{\theta}_{2}\right)\right)$ is continuous on the parameters $\boldsymbol{\theta}_{2}$, it can be shown that $I_{2}=o_{p}(1)$ and $I_{3}=o_{p}(1)$. For $I_{4}$, since the samples are i.i.d. and $\bar{K}\left(v_{0}, \gamma_{0}\right), \varsigma_{\lambda_{n}, i}\left(\boldsymbol{\theta}_{1, \tau}\right), \frac{1}{\tau} G_{s p}^{\prime}\left(e_{i}\left(\boldsymbol{\theta}_{2, \tau}\right)\right)$ and $E\left[\left\|W_{i} W_{i}^{\top}\right\| \mid T=c\right]$ are bounded, it is easy to see that $I_{4}=o_{p}(1)$. Let $Y-q\left(\boldsymbol{\theta}_{1, \tau}\right)=\varepsilon_{\tau}^{q}$ and then $f_{Y \mid W, T=c}\left(q\left(\boldsymbol{\theta}_{1, \tau}\right)\right)=f_{\varepsilon_{\tau}^{q} \mid W, T=c}(0)$. For $I_{5}$, at first notice that as $\lambda_{n}=o(1)$,

$$
\begin{aligned}
\left|E\left[\varsigma_{\lambda_{n}}\left(\boldsymbol{\theta}_{1, \tau}\right) \mid W, T=c\right]-f_{\varepsilon_{\tau}^{q} \mid W, T=c}(0)\right| & =\left|E\left[\frac{1\left\{\left|Y_{i}-q_{i}\left(\boldsymbol{\theta}_{1, \tau}\right)\right| \leq \lambda_{n}\right\}}{2 \lambda_{n}} \cdot \mid W, T=c o\right]-f_{\varepsilon_{\tau}^{q} \mid W, T=c o}(0)\right| \\
& =\left|\frac{1}{2 \lambda_{n}} \int_{-\lambda_{n}}^{\lambda_{n}} f_{\varepsilon_{\tau}^{q} \mid W, T=c o}(a) d a-f_{\varepsilon_{\tau}^{q} \mid W, T=c o}(0)\right| \\
& \leq\left|\frac{1}{2 \lambda_{n}} \times 2 \lambda_{n} f_{\varepsilon_{\tau}^{q} \mid W, T=c o}\left(a^{*}\right)-f_{\varepsilon_{\tau}^{q} \mid W, T=c o}(0)\right|, \\
& =o_{p}(1),
\end{aligned}
$$

where $f_{\mathcal{E}_{\tau}^{q} \mid W, T=c o}\left(a^{*}\right)=\max _{a \in\left[-\lambda_{n}, \lambda_{n}\right]} f_{\mathcal{E}_{\tau}^{q} \mid W, T=c o}(a)$. Thus

$$
\lim _{\lambda_{n} \rightarrow 0} E\left[\varsigma_{\lambda_{n}}\left(\boldsymbol{\theta}_{1, \tau}\right) \mid W, T=c\right]=f_{Y \mid W, T=c}\left(q\left(\boldsymbol{\theta}_{1, \tau}\right)\right) .
$$

Furthermore,

$$
\begin{aligned}
\lim _{\lambda_{n} \rightarrow 0} E\left[\bar{K}\left(v_{0}, \gamma_{0}\right) \varsigma_{\lambda_{n}}\left(\boldsymbol{\theta}_{1, \tau}\right) \frac{1}{\tau} G_{s p}^{\prime}\left(e\left(\boldsymbol{\theta}_{2, \tau}\right)\right) W W^{\top}\right]= & \lim _{\lambda_{n} \rightarrow 0} E\left[\varsigma_{\lambda_{n}}\left(\boldsymbol{\theta}_{1, \tau}\right) \frac{1}{\tau} G_{s p}^{\prime}\left(e\left(\boldsymbol{\theta}_{2, \tau}\right)\right) W W^{\top} \mid T=c\right] \times P(T=c) \\
= & E\left[\lim _{\lambda_{n} \rightarrow 0} E\left[\varsigma_{\lambda_{n}}\left(\boldsymbol{\theta}_{1, \tau}\right) \mid W, T=c\right] \frac{1}{\tau} G_{s p}^{\prime}\left(e\left(\boldsymbol{\theta}_{2, \tau}\right)\right)\right. \\
& \left.\times W W^{\top} \mid T=c\right] \times P(T=c) \\
= & E\left[f_{Y \mid W, T=c}\left(q\left(\boldsymbol{\theta}_{1, \tau}\right)\right) \frac{1}{\tau} G_{s p}^{\prime}\left(e\left(\boldsymbol{\theta}_{2, \tau}\right)\right)\right. \\
& \left.\times W W^{\top} \mid T=c\right] \times P(T=c) \\
= & E\left[\bar{K}\left(v_{0}, \gamma_{0}\right) f_{Y \mid W, T=c o}\left(q\left(\boldsymbol{\theta}_{1, \tau}\right)\right) \frac{1}{\tau} G_{s p}^{\prime}\left(e\left(\boldsymbol{\theta}_{2, \tau}\right)\right) W W^{\top}\right] \\
= & C_{\tau, 11}^{s p *} .
\end{aligned}
$$


Thus $I_{5}=o(1)$. Combining the above results, we can conclude that $\hat{\mathbf{C}}_{\tau, 11}^{s p *} \stackrel{p}{\rightarrow} \mathbf{C}_{\tau, 11}^{s p *}$.

\section{A.3 Local Lipschitz continuity.}

Let $\boldsymbol{\vartheta}_{\boldsymbol{\theta}_{1}}(\tau):=\left\{\boldsymbol{\theta}_{1}:\left\|\boldsymbol{\theta}_{1}-\boldsymbol{\theta}_{1, \tau}\right\|<\varepsilon_{1}\right\}$ and $\boldsymbol{\vartheta}_{\boldsymbol{\theta}_{2}}(\tau):=\left\{\boldsymbol{\theta}_{2}:\left\|\boldsymbol{\theta}_{2}-\boldsymbol{\theta}_{2, \tau}\right\|<\varepsilon_{2}\right\}$ be neighborhoods of $\boldsymbol{\theta}_{1, \tau}$ and $\boldsymbol{\theta}_{2, \tau}$, and $\boldsymbol{\vartheta}_{\boldsymbol{\theta}}(\tau)=\boldsymbol{\vartheta}_{\boldsymbol{\theta}_{1}}(\tau) \cap \boldsymbol{\vartheta}_{\boldsymbol{\theta}_{2}}(\tau)$. Let $\boldsymbol{\theta}_{1}^{1}$ and $\boldsymbol{\theta}_{1}^{2}$ be two different vectors of parameter values in $\boldsymbol{\vartheta}_{\boldsymbol{\theta}_{1}}(\tau)$ and $\boldsymbol{\theta}_{2}^{1}$ and $\boldsymbol{\theta}_{2}^{2}$ be two different vectors of parameter values in $\boldsymbol{\vartheta}_{\boldsymbol{\theta}_{2}}(\tau)$. Let $\boldsymbol{\theta}^{1}:=\left(\boldsymbol{\theta}_{1}^{1}, \boldsymbol{\theta}_{2}^{1}\right)$ and $\boldsymbol{\theta}^{2}:=\left(\boldsymbol{\theta}_{1}^{2}, \boldsymbol{\theta}_{2}^{2}\right)$. Let

$$
\begin{aligned}
& A_{1, \tau}(V):=\sup _{\boldsymbol{\theta}_{1} \in \boldsymbol{\vartheta}_{\boldsymbol{\theta}_{1}}(\tau)}\left\|G_{1}^{\prime}\left(q\left(\boldsymbol{\theta}_{1}\right)\right) \nabla_{\boldsymbol{\theta}_{1}} q\left(\boldsymbol{\theta}_{1}\right)\right\|, \\
& A_{2, \tau}(V):=\sup _{\boldsymbol{\theta} \in \boldsymbol{\vartheta}_{\boldsymbol{\theta}}(\tau)}\left\|G_{2}^{\prime \prime}\left(e\left(\boldsymbol{\theta}_{2}\right)\right) q\left(\boldsymbol{\theta}_{1}\right) \nabla_{\boldsymbol{\theta}_{2}} e\left(\boldsymbol{\theta}_{2}\right)\right\|, \\
& A_{3, \tau}(V):=\sup _{\boldsymbol{\theta} \in \boldsymbol{\vartheta}_{\boldsymbol{\theta}}(\tau)}\left\|G_{2}^{\prime}\left(e\left(\boldsymbol{\theta}_{2}\right)\right) \nabla_{\boldsymbol{\theta}_{1}} q\left(\boldsymbol{\theta}_{1}\right)\right\|, \\
& A_{4, \tau}(V):=\sup _{\boldsymbol{\theta}_{2} \in \boldsymbol{\vartheta}_{\boldsymbol{\theta}_{2}}(\tau)}\left\|Y G_{2}^{\prime \prime}\left(e\left(\boldsymbol{\theta}_{2}\right)\right) \nabla_{\boldsymbol{\theta}_{2}} e\left(\boldsymbol{\theta}_{2}\right)\right\|, \\
& A_{5, \tau}(V):=\sup _{\boldsymbol{\theta} \in \boldsymbol{\vartheta}_{\boldsymbol{\theta}}(\tau)}\left\|G_{2}^{\prime \prime}\left(e\left(\boldsymbol{\theta}_{2}\right)\right)\left(e\left(\boldsymbol{\theta}_{2}\right)-q\left(\boldsymbol{\theta}_{1}\right)\right) \nabla_{\boldsymbol{\theta}_{2}} e\left(\boldsymbol{\theta}_{2}\right)\right\|,
\end{aligned}
$$

where $V=(Y, W)$.

Lemma 2 (Local Lipschitz continuity) If Assumptions 2.4 and 2.5 hold, for all $\left(\boldsymbol{\theta}_{1}^{1}, \boldsymbol{\theta}_{1}^{2}\right) \in \boldsymbol{\vartheta}_{\boldsymbol{\theta}_{1}}(\tau)$ and $\left(\boldsymbol{\theta}_{2}^{1}, \boldsymbol{\theta}_{2}^{2}\right) \in \boldsymbol{\vartheta}_{\boldsymbol{\theta}_{2}}(\tau)$, given $V=(Y, W)$, there exists a constant $A_{\tau}^{*}(V)$ such that

$$
\left|F Z_{\tau}\left(q\left(\boldsymbol{\theta}_{1}^{1}\right), e_{1}\left(\boldsymbol{\theta}_{2}^{1}\right), Y\right)-F Z_{\tau}\left(q\left(\boldsymbol{\theta}_{1}^{2}\right), e_{1}\left(\boldsymbol{\theta}_{2}^{2}\right), Y\right)\right| \leq A_{\tau}^{*}(V)\left\|\boldsymbol{\theta}^{1}-\boldsymbol{\theta}^{2}\right\|
$$

Proof. To ease the notation, we let $q_{1}:=q\left(\boldsymbol{\theta}_{1}^{1}\right), q_{2}:=q\left(\boldsymbol{\theta}_{1}^{2}\right), e_{1}:=e\left(\boldsymbol{\theta}_{2}^{1}\right)$, and $e_{2}:=q\left(\boldsymbol{\theta}_{2}^{2}\right)$. We rewrite $F Z_{\tau}(q, e, y)$ as

$$
\begin{aligned}
F Z_{\tau}(q, e, y)= & 0.5\left[\left|G_{1}(q)-G_{1}(y)\right|+G_{1}(q)-G_{1}(y)\right]-\tau G_{1}(q) \\
& +\frac{1}{\tau} G_{2}^{\prime}(e)\{0.5[|q-y|+q-y]+\tau(e-q)\} \\
& -G_{2}(e)+\eta(y) .
\end{aligned}
$$

Then

$$
\begin{aligned}
F Z_{\tau}\left(q_{1}, e_{1}, Y\right)-F Z_{\tau}\left(q_{2}, e_{2}, Y\right)= & 0.5\left[\left|G_{1}\left(q_{1}\right)-G_{1}(Y)\right|+G_{1}\left(q_{1}\right)-\left|G_{1}\left(q_{2}\right)-G_{1}(Y)\right|-G_{1}\left(q_{2}\right)\right] \\
& -\tau\left(G_{1}\left(q_{1}\right)-G_{1}\left(q_{2}\right)\right) \\
& +\frac{1}{2 \tau}\left(G_{2}^{\prime}\left(e_{1}\right)\left[\left|q_{1}-Y\right|+q_{1}\right]-G_{2}^{\prime}\left(e_{2}\right)\left[\left|q_{2}-Y\right|+q_{2}\right]\right) \\
& -\frac{Y}{2 \tau}\left[G_{2}^{\prime}\left(e_{1}\right)-G_{2}^{\prime}\left(e_{2}\right)\right] \\
& +\left[G_{2}^{\prime}\left(e_{1}\right)\left(e_{1}-q_{1}\right)-G_{2}^{\prime}\left(e_{2}\right)\left(e_{2}-q_{2}\right)\right. \\
& \left.-\left(G_{2}\left(e_{1}\right)-G_{2}\left(e_{2}\right)\right)\right]
\end{aligned}
$$

We next prove that the absolute difference $\left|F Z_{\tau}\left(q_{1}, e_{1}, Y\right)-F Z_{\tau}\left(q_{2}, e_{2}, Y\right)\right|$ is bounded by some functions. Without loss of generality, we assume $q_{1} \geq q_{2}$. The results for $q_{1}<q_{2}$ can be proved in a similar manner. We divide the proof into three cases. 
Cases 1. Suppose $q_{1} \geq q_{2}>Y$. Since $G_{1}($.$) is increasing,$

$$
\begin{aligned}
F Z_{\tau}\left(q_{1}, e_{1}, Y\right)-F Z_{\tau}\left(q_{2}, e_{2}, Y\right)= & (1-\tau)\left[G_{1}\left(q_{1}\right)-G_{1}\left(q_{2}\right)\right] \\
& +\frac{1}{\tau}\left[G_{2}^{\prime}\left(e_{1}\right) q_{1}-G_{2}^{\prime}\left(e_{2}\right) q_{2}\right] \\
& -\frac{Y}{\tau}\left[G_{2}^{\prime}\left(e_{1}\right)-G_{2}^{\prime}\left(e_{2}\right)\right] \\
& +\left[G_{2}^{\prime}\left(e_{1}\right)\left(e_{1}-q_{1}\right)-G_{2}^{\prime}\left(e_{2}\right)\left(e_{2}-q_{2}\right)\right. \\
& \left.-\left(G_{2}\left(e_{1}\right)-G_{2}\left(e_{2}\right)\right)\right] .
\end{aligned}
$$

And

$$
\begin{aligned}
\left|F Z_{\tau}\left(q_{1}, e_{1}, Y\right)-F Z_{\tau}\left(q_{2}, e_{2}, Y\right)\right| \leq & \left|G_{1}\left(q_{1}\right)-G_{1}\left(q_{2}\right)\right| \\
& +\frac{1}{\tau}\left|G_{2}^{\prime}\left(e_{1}\right) q_{1}-G_{2}^{\prime}\left(e_{2}\right) q_{2}\right| \\
& +\frac{1}{\tau}\left|Y\left[G_{2}^{\prime}\left(e_{1}\right)-G_{2}^{\prime}\left(e_{2}\right)\right]\right| \\
& +\mid G_{2}^{\prime}\left(e_{1}\right)\left(e_{1}-q_{1}\right)-G_{2}^{\prime}\left(e_{2}\right)\left(e_{2}-q_{2}\right) \\
& -\left(G_{2}\left(e_{1}\right)-G_{2}\left(e_{2}\right)\right) \mid,
\end{aligned}
$$

Case 2. Suppose $q_{1} \geq Y \geq q_{2}$. Since $G_{1}($.$) is increasing,$

$$
\begin{aligned}
F Z_{\tau}\left(q_{1}, e_{1}, Y\right)-F Z_{\tau}\left(q_{2}, e_{2}, Y\right)= & G_{1}\left(q_{1}\right)-G_{1}(Y) \\
& -\tau\left(G_{1}\left(q_{1}\right)-G_{1}\left(q_{2}\right)\right) \\
& +\frac{1}{\tau}\left[G_{2}^{\prime}\left(e_{1}\right) q_{1}-G_{2}^{\prime}\left(e_{1}\right) Y\right] \\
& +\left[G_{2}^{\prime}\left(e_{1}\right)\left(e_{1}-q_{1}\right)-G_{2}^{\prime}\left(e_{2}\right)\left(e_{2}-q_{2}\right)\right. \\
& \left.-\left(G_{2}\left(e_{1}\right)-G_{2}\left(e_{2}\right)\right)\right] .
\end{aligned}
$$

By $G_{2}^{\prime}\left(e_{1}\right) \geq 0, G_{2}^{\prime}\left(e_{1}\right) Y \geq G_{2}^{\prime}\left(e_{1}\right) q_{2}$ and $G_{2}^{\prime}\left(e_{1}\right) q_{1}-G_{2}^{\prime}\left(e_{1}\right) Y \leq G_{2}^{\prime}\left(e_{1}\right) q_{1}-G_{2}^{\prime}\left(e_{1}\right) q_{2}$. Again since $G_{1}\left(q_{1}\right), G_{1}(Y) \geq G_{1}\left(q_{2}\right)$ and $G_{1}\left(q_{1}\right)-G_{1}(Y) \leq G_{1}\left(q_{1}\right)-G_{1}\left(q_{2}\right)$,

$$
\begin{aligned}
\left|F Z_{\tau}\left(q_{1}, e_{1}, Y\right)-F Z_{\tau}\left(q_{2}, e_{2}, Y\right)\right| \leq \quad & \left|G_{1}\left(q_{1}\right)-G_{1}\left(q_{2}\right)\right| \\
& +\frac{1}{\tau}\left|G_{2}^{\prime}\left(e_{1}\right) q_{1}-G_{2}^{\prime}\left(e_{2}\right) q_{2}\right| \\
& +\mid G_{2}^{\prime}\left(e_{1}\right)\left(e_{1}-q_{1}\right)-G_{2}^{\prime}\left(e_{2}\right)\left(e_{2}-q_{2}\right) \\
& -\left(G_{2}\left(e_{1}\right)-G_{2}\left(e_{2}\right)\right) \mid .
\end{aligned}
$$

Case 3. Suppose $Y>q_{1} \geq q_{2}$. Since $G_{1}$ (.) is increasing,

$$
\begin{aligned}
F Z_{\tau}\left(q_{1}, e_{1}, Y\right)-F Z_{\tau}\left(q_{2}, e_{2}, Y\right)= & -\tau\left(G_{1}\left(q_{1}\right)-G_{1}\left(q_{2}\right)\right) \\
& +\left[G_{2}^{\prime}\left(e_{1}\right)\left(e_{1}-q_{1}\right)-G_{2}^{\prime}\left(e_{2}\right)\left(e_{2}-q_{2}\right)\right. \\
& \left.-\left(G_{2}\left(e_{1}\right)-G_{2}\left(e_{2}\right)\right)\right] .
\end{aligned}
$$

And

$$
\begin{aligned}
\left|F Z_{\tau}\left(q_{1}, e_{1}, Y\right)-F Z_{\tau}\left(q_{2}, e_{2}, Y\right)\right| \leq & \tau\left|G_{1}\left(q_{1}\right)-G_{1}\left(q_{2}\right)\right| \\
& +\mid G_{2}^{\prime}\left(e_{1}\right)\left(e_{1}-q_{1}\right)-G_{2}^{\prime}\left(e_{2}\right)\left(e_{2}-q_{2}\right) \\
& -\left(G_{2}\left(e_{1}\right)-G_{2}\left(e_{2}\right)\right) \mid .
\end{aligned}
$$


For case 1, if Assumptions 2.4 and 2.5 hold, given $(Y, W)$ and $\tau \in(0,1)$, we can have the following results for the terms in the right hand side of 42 :

$$
\begin{gathered}
\left|G_{1}\left(q_{1}\right)-G_{1}\left(q_{2}\right)\right| \leq A_{1, \tau}(V)\left\|\boldsymbol{\theta}_{1}^{1}-\boldsymbol{\theta}_{1}^{2}\right\| \leq A_{1, \tau}(V)\left\|\boldsymbol{\theta}^{1}-\boldsymbol{\theta}^{2}\right\|, \\
\frac{1}{\tau}\left|G_{2}^{\prime}\left(e_{1}\right) q_{1}-G_{2}^{\prime}\left(e_{2}\right) q_{2}\right| \leq \frac{1}{\tau}\left[A_{2, \tau}(V)+A_{3, \tau}(V)\right]\left\|\boldsymbol{\theta}^{1}-\boldsymbol{\theta}^{2}\right\|, \\
\frac{1}{\tau}\left|Y\left[G_{2}^{\prime}\left(e_{1}\right)-G_{2}^{\prime}\left(e_{2}\right)\right]\right| \leq A_{4, \tau}(V)\left\|\boldsymbol{\theta}_{2}^{1}-\boldsymbol{\theta}_{2}^{2}\right\| \leq A_{4, \tau}(V)\left\|\boldsymbol{\theta}^{1}-\boldsymbol{\theta}^{2}\right\|, \\
\left|G_{2}^{\prime}\left(e_{1}\right)\left(e_{1}-q_{1}\right)-G_{2}^{\prime}\left(e_{2}\right)\left(e_{2}-q_{2}\right)-\left(G_{2}\left(e_{1}\right)-G_{2}\left(e_{2}\right)\right)\right| \leq\left[A_{2, \tau}(V)+A_{5, \tau}(V)\right]\left\|\boldsymbol{\theta}^{1}-\boldsymbol{\theta}^{2}\right\| .
\end{gathered}
$$

Summing the above inequalities together, we can conclude that given $(Y, W), F Z_{\tau}\left(q\left(\boldsymbol{\theta}_{1}\right), e_{1}\left(\boldsymbol{\theta}_{2}\right), Y\right)$ is locally Lipschitz continuous in $\boldsymbol{\theta}$ around $\boldsymbol{\theta}_{\tau}$. Using the above results for case 1 , we can also prove that if Assumptions 2.4 and 2.5 hold, $F Z_{\tau}\left(q\left(\boldsymbol{\theta}_{1}\right), e_{1}\left(\boldsymbol{\theta}_{2}\right), Y\right)$ is also locally Lipschitz continuous in $\boldsymbol{\theta}$ around $\boldsymbol{\theta}_{\tau}$ for cases 2 and 3 .

\section{A.4 Global Lipschitz continuity}

Recall that

$$
\begin{aligned}
F Z_{\tau}^{s p}\left(q\left(\boldsymbol{\theta}_{1}\right), e\left(\boldsymbol{\theta}_{2}\right), y\right)= & \frac{\exp \left(e\left(\boldsymbol{\theta}_{2}\right)\right)}{1+\exp \left(e\left(\boldsymbol{\theta}_{2}\right)\right)}\left[e\left(\boldsymbol{\theta}_{2}\right)+\frac{1}{\tau} \max \left(q\left(\boldsymbol{\theta}_{1}\right)-y, 0\right)-q\left(\boldsymbol{\theta}_{1}\right)\right] \\
& +\ln (1+\exp (y))-\ln \left(1+\exp \left(e\left(\boldsymbol{\theta}_{2}\right)\right)\right),
\end{aligned}
$$

and

$$
h(\tau, \boldsymbol{\theta}):=\tau\left[F Z_{\tau}^{s p}\left(q\left(\boldsymbol{\theta}_{1}\right), e\left(\boldsymbol{\theta}_{2}\right), Y\right)-\ln (1+\exp (Y))\right]
$$

Let $\mathcal{T}:=(0,1)$ and

$$
\begin{aligned}
B_{1}(V):= & \sup _{\boldsymbol{\theta}_{1} \in \mathbf{\Theta}_{1}}\left|q\left(\boldsymbol{\theta}_{1}\right)\right|, \\
B_{2}(V):= & \sup _{\boldsymbol{\theta} \in \boldsymbol{\Theta}}\left\|q\left(\boldsymbol{\theta}_{1}\right) \nabla_{\boldsymbol{\theta}_{2}} e\left(\boldsymbol{\theta}_{2}\right)\right\|, \\
B_{3}(V):= & \sup _{\boldsymbol{\theta} \in \boldsymbol{\Theta}_{1}}\left\|\nabla_{\boldsymbol{\theta}_{1}} q\left(\boldsymbol{\theta}_{1}\right)\right\|, \\
B_{4}(V) & :=\sup _{\boldsymbol{\theta}_{2} \in \mathbf{\Theta}_{2}}\left\|Y \nabla_{\boldsymbol{\theta}_{2}} e\left(\boldsymbol{\theta}_{2}\right)\right\|, \\
B_{5}(V) & :=\sup _{\boldsymbol{\theta} \in \boldsymbol{\Theta}}\left\|\left(e\left(\boldsymbol{\theta}_{2}\right)-q\left(\boldsymbol{\theta}_{1}\right)\right) \nabla_{\boldsymbol{\theta}_{2}} e\left(\boldsymbol{\theta}_{2}\right)\right\| .
\end{aligned}
$$

Let $\boldsymbol{\theta}^{1}:=\left(\boldsymbol{\theta}_{1}^{1}, \boldsymbol{\theta}_{2}^{1}\right)$ and $\boldsymbol{\theta}^{2}:=\left(\boldsymbol{\theta}_{1}^{2}, \boldsymbol{\theta}_{2}^{2}\right)$ be two vectors of parameter values.

Lemma 3 (Global Lipschitz continuity) If Assumption 2.4 holds, for all $\left(\tau_{1}, \tau_{2}\right) \in \mathcal{T},\left(\boldsymbol{\theta}_{1}^{1}, \boldsymbol{\theta}_{1}^{2}\right) \in \boldsymbol{\Theta}_{1}$ and $\left(\boldsymbol{\theta}_{2}^{1}, \boldsymbol{\theta}_{2}^{2}\right) \in \boldsymbol{\Theta}_{2}$, given $V=(Y, W)$, there exists a constant $B_{\tau}^{*}(V)$ such that

$$
\left|h\left(\tau_{1}, \boldsymbol{\theta}^{1}\right)-h\left(\tau_{2}, \boldsymbol{\theta}^{2}\right)\right| \leq B_{\tau}^{*}(V)\left(\left\|\tau_{1}-\tau_{2}\right\|+\left\|\boldsymbol{\theta}^{1}-\boldsymbol{\theta}^{2}\right\|\right)
$$

Proof. We use similar notations and strategy as in proving Lemma 2. At first note that $h(\tau, \boldsymbol{\theta})$ can be rewritten as

$$
\begin{aligned}
h(\tau, \boldsymbol{\theta})= & \frac{\exp \left(e\left(\boldsymbol{\theta}_{2}\right)\right)}{1+\exp \left(e\left(\boldsymbol{\theta}_{2}\right)\right)}\left\{0.5\left[\left|q\left(\boldsymbol{\theta}_{1}\right)-Y\right|+q\left(\boldsymbol{\theta}_{1}\right)-Y\right]+\tau\left(e\left(\boldsymbol{\theta}_{2}\right)-q\left(\boldsymbol{\theta}_{1}\right)\right)\right\} \\
& -\tau \ln \left(1+\exp \left(e\left(\boldsymbol{\theta}_{2}\right)\right)\right) .
\end{aligned}
$$


Then

$$
\begin{aligned}
h\left(\tau_{1}, \boldsymbol{\theta}^{1}\right)-h\left(\tau_{2}, \boldsymbol{\theta}^{2}\right)= & \frac{0.5 \exp \left(e_{1}\right)}{1+\exp \left(e_{1}\right)}\left[\left|q_{1}-Y\right|+q_{1}-Y\right]-\frac{0.5 \exp \left(e_{2}\right)}{1+\exp \left(e_{2}\right)}\left[\left|q_{2}-Y\right|+q_{2}-Y\right] \\
& +\left[\frac{\tau_{1} \exp \left(e_{1}\right)}{1+\exp \left(e_{1}\right)}\left(e_{1}-q_{1}\right)-\frac{\tau_{2} \exp \left(e_{2}\right)}{1+\exp \left(e_{2}\right)}\left(e_{2}-q_{2}\right)\right. \\
& \left.-\left(\tau_{1} \ln \left(1+\exp \left(e_{1}\right)\right)-\tau_{2} \ln \left(1+\exp \left(e_{2}\right)\right)\right)\right]
\end{aligned}
$$

We assume $q_{1} \geq q_{2}$ and $\tau_{1} \geq \tau_{2}$. The results for $q_{1}<q_{2}$ and $\tau_{1}<\tau_{2}$ can be proved in a similar manner. We separate the proof into three cases.

Cases 1. Suppose $q_{1} \geq q_{2}>Y$. It can be shown that

$$
\begin{aligned}
\left|h\left(\tau_{1}, \boldsymbol{\theta}^{1}\right)-h\left(\tau_{2}, \boldsymbol{\theta}^{2}\right)\right| \leq & \left|\frac{\exp \left(e_{1}\right)}{1+\exp \left(e_{1}\right)} q_{1}-\frac{\exp \left(e_{2}\right)}{1+\exp \left(e_{2}\right)} q_{2}\right| \\
& +\left|Y \frac{\exp \left(e_{1}\right)}{1+\exp \left(e_{1}\right)}-Y \frac{\exp \left(e_{2}\right)}{1+\exp \left(e_{2}\right)}\right| \\
& +\mid \frac{\tau_{1} \exp \left(e_{1}\right)}{1+\exp \left(e_{1}\right)}\left(e_{1}-q_{1}\right)-\frac{\tau_{2} \exp \left(e_{2}\right)}{1+\exp \left(e_{2}\right)}\left(e_{2}-q_{2}\right) \\
& -\left(\tau_{1} \ln \left(1+\exp \left(e_{1}\right)\right)-\tau_{2} \ln \left(1+\exp \left(e_{2}\right)\right)\right) \mid
\end{aligned}
$$

Case 2. Suppose $q_{1} \geq Y \geq q_{2}$. It can be shown that

$$
\begin{aligned}
\left|h\left(\tau_{1}, \boldsymbol{\theta}^{1}\right)-h\left(\tau_{2}, \boldsymbol{\theta}^{2}\right)\right| \leq & \left|\frac{\exp \left(e_{1}\right)}{1+\exp \left(e_{1}\right)} q_{1}-\frac{\exp \left(e_{2}\right)}{1+\exp \left(e_{2}\right)} q_{2}\right| \\
& +\mid \frac{\tau_{1} \exp \left(e_{1}\right)}{1+\exp \left(e_{1}\right)}\left(e_{1}-q_{1}\right)-\frac{\tau_{2} \exp \left(e_{2}\right)}{1+\exp \left(e_{2}\right)}\left(e_{2}-q_{2}\right) \\
& -\left(\tau_{1} \ln \left(1+\exp \left(e_{1}\right)\right)-\tau_{2} \ln \left(1+\exp \left(e_{2}\right)\right)\right) \mid
\end{aligned}
$$

Case 3. Suppose $Y>q_{1} \geq q_{2}$. It can be shown that

$$
\begin{aligned}
\left|h\left(\tau_{1}, \boldsymbol{\theta}^{1}\right)-h\left(\tau_{2}, \boldsymbol{\theta}^{2}\right)\right| \leq & +\mid \frac{\tau_{1} \exp \left(e_{1}\right)}{1+\exp \left(e_{1}\right)}\left(e_{1}-q_{1}\right)-\frac{\tau_{2} \exp \left(e_{2}\right)}{1+\exp \left(e_{2}\right)}\left(e_{2}-q_{2}\right) \\
& -\left(\tau_{1} \ln \left(1+\exp \left(e_{1}\right)\right)-\tau_{2} \ln \left(1+\exp \left(e_{2}\right)\right)\right) \mid
\end{aligned}
$$

Let $(\bar{\tau}, \bar{q}, \bar{e})$ be some middle point between $\left(\tau_{1}, q_{1}, e_{1}\right)$ and $\left(\tau_{2}, q_{2}, e_{2}\right)$. Using the mean value theorem, it is straightforward to show that

$$
\begin{aligned}
\left|\frac{\exp \left(e_{1}\right)}{1+\exp \left(e_{1}\right)} q_{1}-\frac{\exp \left(e_{2}\right)}{1+\exp \left(e_{2}\right)} q_{2}\right| \leq & \left|\frac{\exp (\bar{e})}{1+\exp (\bar{e})}\right|\left|q_{1}-q_{2}\right| \\
& +\left|\frac{\exp (\bar{e})}{1+\exp (\bar{e})}\left(1-\frac{\exp (\bar{e})}{1+\exp (\bar{e})}\right)\right|\left|\bar{q}\left(e_{1}-e_{2}\right)\right| \\
\leq & \left|q_{1}-q_{2}\right|+\left|\bar{q}\left(e_{1}-e_{2}\right)\right|, \\
\left|Y \frac{\exp \left(e_{1}\right)}{1+\exp \left(e_{1}\right)}-Y \frac{\exp \left(e_{2}\right)}{1+\exp \left(e_{2}\right)}\right| \leq & \left|\frac{\exp (\bar{e})}{1+\exp (\bar{e})}\left(1-\frac{\exp (\bar{e})}{1+\exp (\bar{e})}\right)\right|\left|Y\left(e_{1}-e_{2}\right)\right| \\
\leq & \left|Y\left(e_{1}-e_{2}\right)\right|,
\end{aligned}
$$


and

$$
\begin{aligned}
\mid \frac{\tau_{1} \exp \left(e_{1}\right)}{1+\exp \left(e_{1}\right)}\left(e_{1}-q_{1}\right)-\frac{\tau_{2} \exp \left(e_{2}\right)}{1+\exp \left(e_{2}\right)}\left(e_{2}-q_{2}\right) & \\
-\left(\tau_{1} \ln \left(1+\exp \left(e_{1}\right)\right)-\tau_{2} \ln \left(1+\exp \left(e_{2}\right)\right)\right) \mid \leq & \left|\frac{\exp (\bar{e})}{1+\exp (\bar{e})} \bar{e}-\ln (1+\exp (\bar{e}))\right|\left|\tau_{1}-\tau_{2}\right| \\
& +\left|\frac{\exp (\bar{e}) \bar{q}}{1+\exp (\bar{e})}\right|\left|\tau_{1}-\tau_{2}\right|+\left|\frac{\bar{\tau} \exp (\bar{e})}{1+\exp (\bar{e})}\right|\left|q_{1}-q_{2}\right| \\
& +\left|\frac{\bar{\tau} \exp (\bar{e})}{1+\exp (\bar{e})}\left(1-\frac{\exp (\bar{e})}{1+\exp (\bar{e})}\right)\right| \\
& \times\left|(\bar{e}-\bar{q})\left(e_{1}-e_{2}\right)\right| \\
\leq & (\ln 2+|\bar{q}|)\left|\tau_{1}-\tau_{2}\right|+\left|q_{1}-q_{2}\right| \\
& +\left|(\bar{e}-\bar{q})\left(e_{1}-e_{2}\right)\right|
\end{aligned}
$$

To see why 47 holds, let $\varpi(x)=\exp (x)(1+\exp (x))^{-1} x-\ln (1+\exp (x))$. It can be shown that $\varpi(x)$ is monotonically decreasing for $x<0$ and monotonically increasing for $x \geq 0$ and $\varpi(0)=-\ln 2$, $\lim _{x \rightarrow \infty} \varpi(x)=0$, and $\lim _{x \rightarrow-\infty} \varpi(x)=0$. Therefore we can conclude that

$$
|\varpi(x)| \leq \ln 2
$$

for all $x \in \mathbb{R}$. If Assumption 2.4 holds, it can be shown for 45 ,

$$
\begin{aligned}
\left|q_{1}-q_{2}\right|+\left|\bar{q}\left(e_{1}-e_{2}\right)\right| & \leq\left|\nabla_{\boldsymbol{\theta}_{1}} q\left(\overline{\boldsymbol{\theta}}_{1}\right)\right|\left\|\boldsymbol{\theta}_{1}^{1}-\boldsymbol{\theta}_{1}^{2}\right\|+\left|q\left(\boldsymbol{\theta}_{1}\right) \nabla_{\boldsymbol{\theta}_{2}} e\left(\boldsymbol{\theta}_{2}\right)\right|\left\|\boldsymbol{\theta}_{2}^{1}-\boldsymbol{\theta}_{2}^{2}\right\| \\
& \leq B_{3}(V)\left\|\boldsymbol{\theta}_{1}^{1}-\boldsymbol{\theta}_{1}^{2}\right\|+B_{2}(V)\left\|\boldsymbol{\theta}_{2}^{1}-\boldsymbol{\theta}_{2}^{2}\right\|,
\end{aligned}
$$

for 46 ,

$$
\left|Y\left(e_{1}-e_{2}\right)\right| \leq B_{4}(V)\left\|\boldsymbol{\theta}_{2}^{1}-\boldsymbol{\theta}_{2}^{2}\right\|
$$

and for 47 ,

$$
\begin{aligned}
(\ln 2+|\bar{q}|)\left|\tau_{1}-\tau_{2}\right|+\left|q_{1}-q_{2}\right|+\left|(\bar{e}-\bar{q})\left(e_{1}-e_{2}\right)\right| \leq & \left(\ln 2+B_{1}(V)\right)\left|\tau_{1}-\tau_{2}\right|+B_{3}(V)\left\|\boldsymbol{\theta}_{1}^{1}-\boldsymbol{\theta}_{1}^{2}\right\| \\
& +B_{5}(V)\left\|\boldsymbol{\theta}_{2}^{1}-\boldsymbol{\theta}_{2}^{2}\right\| .
\end{aligned}
$$

Summing the above inequalities together, given $V=(Y, W)$, we can conclude that

$$
\left|h\left(\tau_{1}, \boldsymbol{\theta}^{1}\right)-h\left(\tau_{2}, \boldsymbol{\theta}^{2}\right)\right| \leq B^{*}(V)\left(\left\|\tau_{1}-\tau_{2}\right\|+\left\|\boldsymbol{\theta}^{1}-\boldsymbol{\theta}^{2}\right\|\right)
$$

where $B^{*}(V)$ is a constant and is determined by a sum of $\ln 2$ and constants $B_{i}(V), i=1, \ldots, 5$.

\section{A.5 Constructing the simultaneous confidence bands (scb) with the bootstrap}

We use the models of linear in parameters of (6) and (7) as an example to illustrate the bootstrap procedures for constructing the simultaneous confidence bands. Let $\boldsymbol{\theta}_{\tau}=\left(\alpha_{1, \tau}, \boldsymbol{\beta}_{1, \tau}^{\top}, \alpha_{2, \tau}, \boldsymbol{\beta}_{2, \tau}^{\top}\right)^{\top}$ be a vector for parameters at the $\tau$-quantile. The bootstrap procedures are summarized as follows. 
1. Draw a bootstrap sample of size $n: W_{1}^{*}, \ldots, W_{n}^{*}$ with replacement, where

$$
W_{i}^{*}=\left(Y_{i}^{*}, D_{i}^{*}, X_{i}^{* \top}, Z_{i}^{*}\right)^{\top}
$$

$i=1, \ldots, n$, is a vector for the $i$ th random draw sample.

2. Re-estimate the weight $\bar{K}$ with the bootstrap sample. Let $\hat{\tilde{K}}_{i}^{*}, i=1, \ldots, n$ denote the bootstrap estimated truncated weight evaluated with $\left(Y_{i}^{*}, D_{i}^{*}, X_{i}^{*}\right)$.

3. With the bootstrap estimated truncated weight $\hat{\bar{K}}_{i}^{*}$, obtain the bootstrap estimated parameters

$$
\hat{\boldsymbol{\theta}}_{\tau, b}^{*}=\left(\hat{\alpha}_{1, \tau, b}^{*}, \hat{\boldsymbol{\beta}}_{1, \tau, b}^{* \top}, \hat{\alpha}_{2, \tau, b}^{*}, \hat{\boldsymbol{\beta}}_{2, \tau, b}^{* \top}\right)^{\top}
$$

for $\tau \in(0,1)$.

4. Repeat procedures 1 to $3 B$ times to obtain $B$ bootstrap estimated parameters $\hat{\boldsymbol{\theta}}_{\tau, 1}^{*}, \ldots, \hat{\boldsymbol{\theta}}_{\tau, B}^{*}$.

With the bootstrap estimated parameters, we use $\hat{\alpha}_{2, \tau}$ as an example to illustrate the procedures to construct the scb as follows.

1. Calculate the bootstrap standard deviation of $\hat{\alpha}_{2, \tau}, \hat{\sigma}_{\hat{\alpha}_{2, \tau}}^{*}$ with the $B$ bootstrap estimates $\left(\hat{\alpha}_{2, \tau, 1}^{*}, \ldots, \hat{\alpha}_{2, \tau, B}^{*}\right)$.

2. Calculate the bootstrap $t$ statistic of $\hat{\alpha}_{2, \tau}$ :

$$
\hat{t}_{\hat{\alpha}_{2, \tau}, b}^{*}=\frac{\sqrt{n}\left(\hat{\alpha}_{2, \tau, b}^{*}-\hat{\alpha}_{2, \tau}\right)}{\hat{\sigma}_{\hat{\alpha}_{2, \tau}}^{*}}
$$

$b=1, \ldots, B$.

3. For each $b=1, \ldots, B$, calculate the maximal absolute bootstrap $t$ statistic for $\tau \in(0,1)$ :

$$
\hat{t}_{\hat{\alpha}_{2}, b}^{*}=\sup _{\tau \in(0,1)}\left|\hat{t}_{\hat{\alpha}_{2, \tau}, b}\right|
$$

4. Let $\hat{t}_{\hat{\alpha}_{2}}^{*(1-g)}$ be the $(1-g)$ th sample quantile of $\hat{t}_{\hat{\alpha}_{2}, 1}^{*}, \ldots, \hat{t}_{\hat{\alpha}_{2}, B}^{*}$. The $1-g$ scb of $\hat{\alpha}_{2, \tau}$ is

$$
\left[\hat{\alpha}_{2, \tau}-\hat{t}_{\hat{\alpha}_{2}}^{*(1-g)} \frac{\hat{\sigma}_{\hat{\alpha}_{2, \tau}}^{*}}{\sqrt{n}}, \hat{\alpha}_{2, \tau}+\hat{t}_{\hat{\alpha}_{2}}^{*(1-g)} \frac{\hat{\sigma}_{\hat{\alpha}_{2, \tau}}^{*}}{\sqrt{n}}\right] .
$$




\section{References}

ABADIE, A. (2003): "Semiparametric instrumental variable estimation of treatment response models," Journal of Econometrics, 113, 231-263.

Abadie, A., J. Angrist, And G. Imbens (2002): "Instrumental Variables Estimates of the Effect of Subsidized Training on the Quantiles of Trainee Earnings," Econometrica, 70, 91-117.

Angrist, J., V. Chernozhukov, And I. Fernández-Val (2006): "Quantile Regression under Misspecification, with an Application to the U.S. Wage Structure," Econometrica, 74, 539-563.

Angrist, J. D., G. W. Imbens, And D. B. Rubin (1996): "Identification of Causal Effects Using Instrumental Variables," Journal of the American Statistical Association, 91, 444-455.

Belloni, A., V. Chernozhukov, I. Fernández-Val, And C. Hansen (2017): "Program Evaluation and Causal Inference With High-Dimensional Data," Econometrica, 85, 233-298.

Chen, Y.-T., Y.-C. Hsu, And H.-J. WAng (2020): "A Stochastic Frontier Model with Endogenous Treatment Status and Mediator," Journal of Business 8 Economic Statistics, 38, 243-256.

Chernozhukov, V., I. Fernández-Val, and B. Melly (2013): "Inference on Counterfactual Distributions," Econometrica, 81, 2205-2268.

Chernozhukov, V. And C. Hansen (2005): "An IV Model of Quantile Treatment Effects," Econometrica, $73,245-261$.

(2006): "Instrumental quantile regression inference for structural and treatment effect models," Journal of Econometrics, 132, 491-525.

(2008): "Instrumental variable quantile regression: A robust inference approach," Journal of Econometrics, 142, 379-398.

Chernozhukov, V., C. Hansen, And M. Jansson (2007): "Inference approaches for instrumental variable quantile regression," Economics Letters, 95, 272-277.

(2009): "Finite sample inference for quantile regression models," Journal of Econometrics, 152, 93-103.

Chou, R. Y., T.-J. YEN, AND Y.-M. YEN (2020): "Forecasting Expected Shortfall and Value-at-Risk with the FZ Loss and Realized Variance Measures," Available at SSRN: https://ssrn.com/abstract=3448882.

Dimitriadis, T. AND S. BAYER (2019): "A joint quantile and expected shortfall regression framework," Electron. J. Statist., 13, 1823-1871.

Engle, R. F. And S. Manganelli (2004): "CAViaR: Conditional Autoregressive Value at Risk by Regression Quantiles," Journal of Business 85 Economic Statistics, 22, 367-381.

Fissler, T. AND J. F. Ziegel (2016): "Higher order elicitability and Osband's principle," The Annals of Statistics, 44, 1680-1707.

Fricke, H., M. Frölich, M. Huber, And M. Lechner (2020): "Endogeneity and non-response bias in treatment evaluation - nonparametric identification of causal effects by instruments," Journal of Applied Econometrics, 35, 481-504. 
FröLICH, M. AND M. Huber (2017): "Direct and indirect treatment effects-causal chains and mediation analysis with instrumental variables," Journal of the Royal Statistical Society: Series B (Statistical Methodology), 79, 1645-1666.

Frölich, M. AND B. Melly (2013): "Unconditional Quantile Treatment Effects Under Endogeneity," Journal of Business and Economic Statistics, 31, 346-357.

Heckman, J. J., J. Smith, and N. Clements (1997): "Making The Most Out Of Programme Evaluations and Social Experiments: Accounting For Heterogeneity in Programme Impacts," The Review of Economic Studies, 64, 487-535.

Hill, J. B. (2015): "Expected Shortfall Estimation and Gaussian Inference for Infinite Variance Time Series," Journal of Financial Econometrics, 13, 1-44.

Imbens, G. And J. Angrist (1994): "Identification and Estimation of Local Average Treatment Effects," Econometrica, 62, 467-75.

Imbens, G. W. And D. B. Rubin (2015): Causal Inference for Statistics, Social, and Biomedical Sciences: An Introduction, Cambridge University Press.

Kianian, B., J. I. Kim, J. P. Fine, and L. Peng (2021): "Causal Proportional Hazards Estimation with a Binary Instrumental Variable," Statistica Sinica, 31, 673-699.

Koenker, R. (2005): Quantile Regression, Econometric Society Monographs, Cambridge University Press.

Koenker, R. And G. Bassett (1978): "Regression Quantiles," Econometrica, 46, 33-50.

Levy, H. (2016): Stochastic Dominance-Investment Decision Making under Uncertainty, Springer, 3 ed.

Linton, O. AND Z. XiaO (2013): "Estimation of and Inference about the expected shortfall for time series with infinite variance," Econometric Theory, 29, 771-807.

Meng, X. and J. W. Taylor (2020): "Estimating Value-at-Risk and Expected Shortfall using the intraday low and range data," European Journal of Operational Research, 280, 191 - 202.

Newey, W. K. (1991): "Uniform Convergence in Probability and Stochastic Equicontinuity," Econometrica, 59, 1161-1167.

(1997): "Convergence rates and asymptotic normality for series estimators," Journal of Econometrics, $79,147-168$.

Newey, W. K. and D. McFadden (1994): "Chapter 36: Large sample estimation and hypothesis testing," in Handbook of Econometrics, Elsevier, vol. 4, 2111-2245.

Patton, A. J., J. F. Ziegel, and R. Chen (2019): "Dynamic semiparametric models for expected shortfall (and Value-at-Risk)," Journal of Econometrics, 211, 388 - 413.

Powell, J. L. (1984): "Least absolute deviations estimation for the censored regression model," Journal of Econometrics, 25, 303-325.

TAYLOR, J. W. (2019): "Forecasting Value at Risk and Expected Shortfall Using a Semiparametric Approach Based on the Asymmetric Laplace Distribution," Journal of Business \& Economic Statistics, 37, $121-133$. 
VAn der VaArT, A. W. (1998): Asymptotic Statistics, Cambridge Series in Statistical and Probabilistic Mathematics, Cambridge University Press.

Wei, B., L. Peng, M.-J. Zhang, and J. P. Fine (2021): "Estimation of causal quantile effects with a binary instrumental variable and censored data," Journal of the Royal Statistical Society: Series B (Statistical Methodology), forthcoming.

WÜthrich, K. (2020): "A Comparison of Two Quantile Models With Endogeneity," Journal of Business \& Economic Statistics, 38, 443-456. 

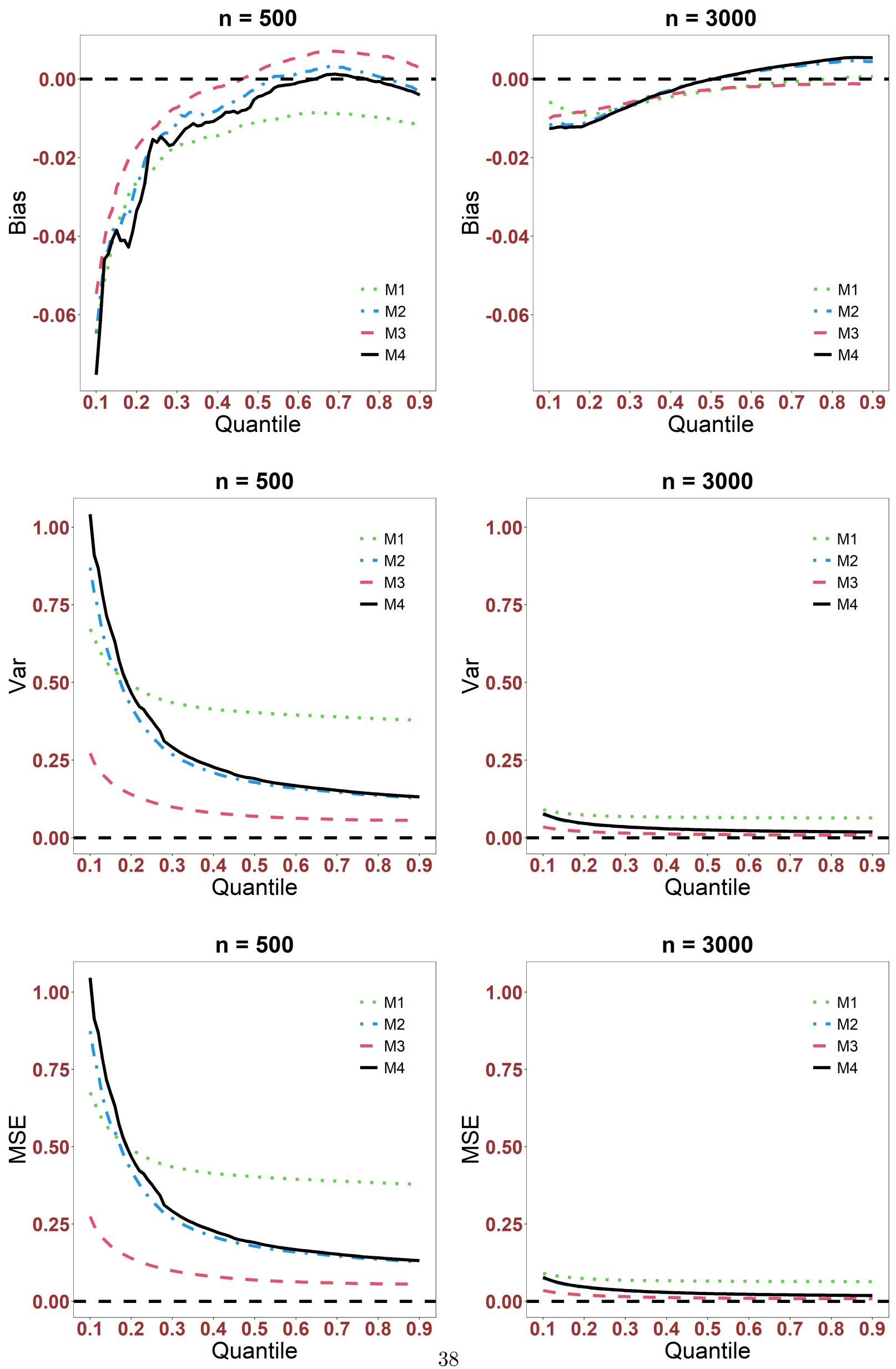

Figure 1: Bias, variance and MSE of the estimated conditional CTATE for the compliers under different quantile levels when $\rho=0$. 

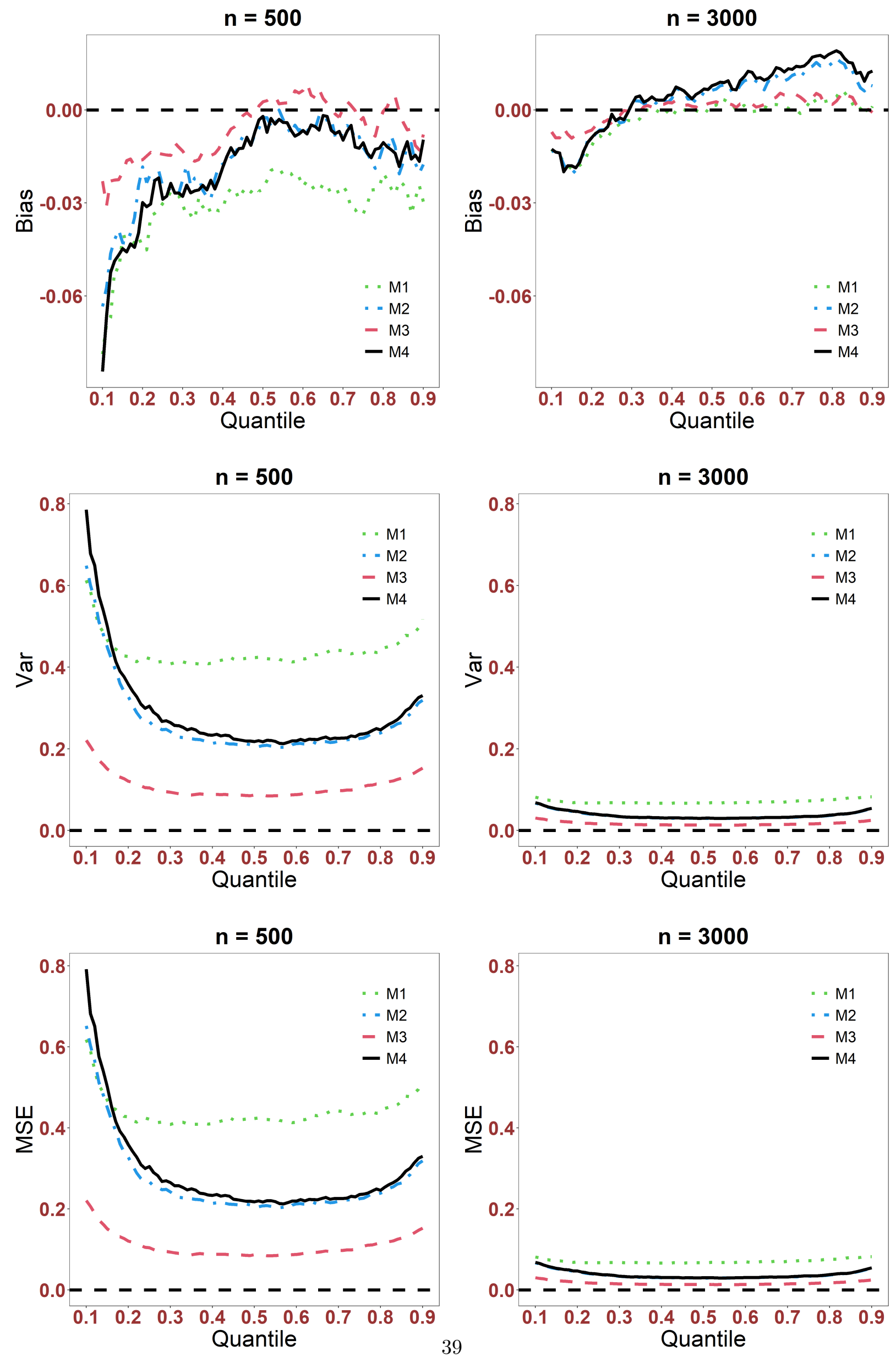

Figure 2: Bias, variance and MSE of the estimated conditional QTE for the compliers under different quantile levels when $\rho=0$. 

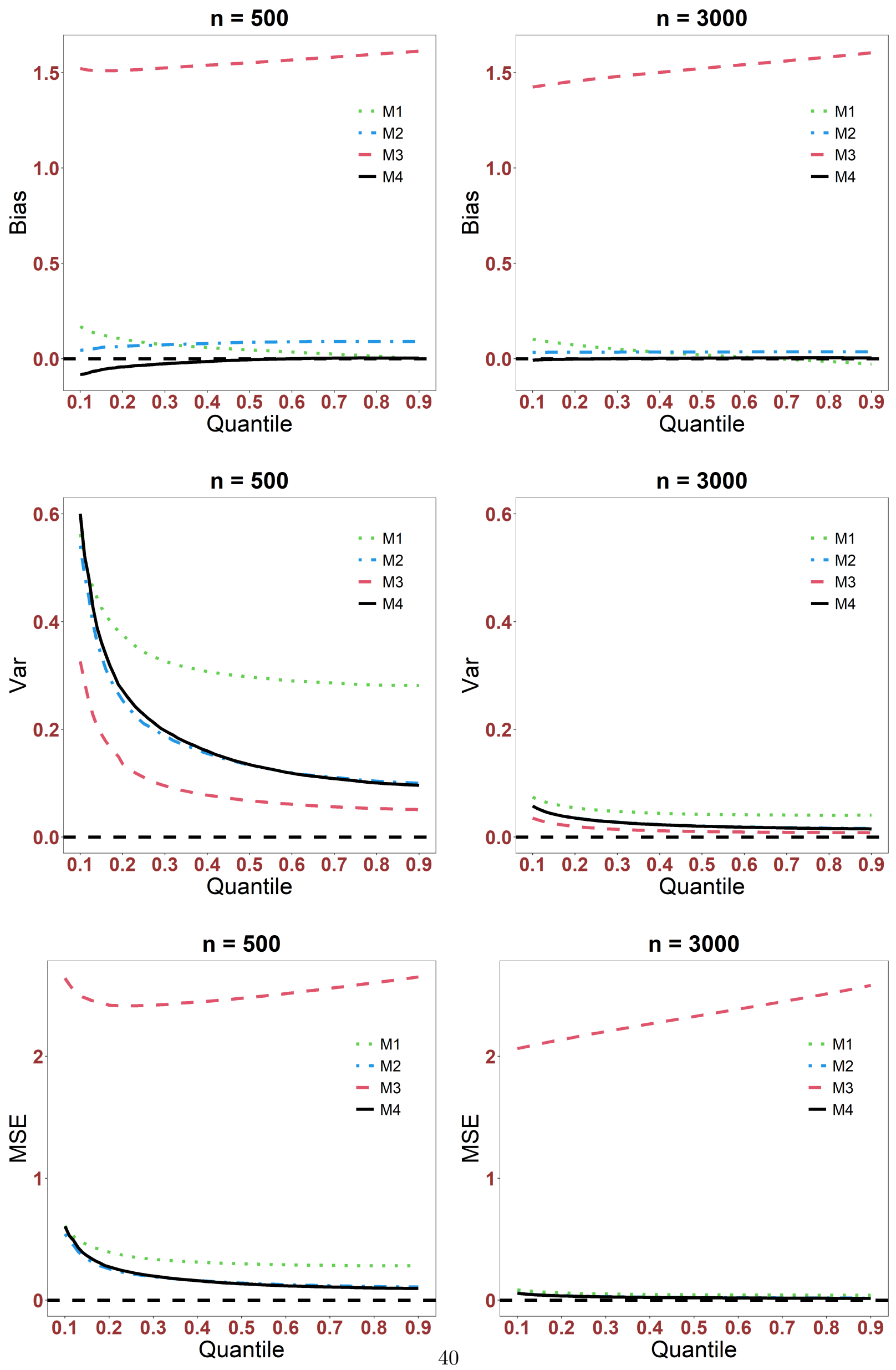

Figure 3: Bias, variance and MSE of the estimated conditional CTATE for the compliers under different quantile levels when $\rho=0.5$. 

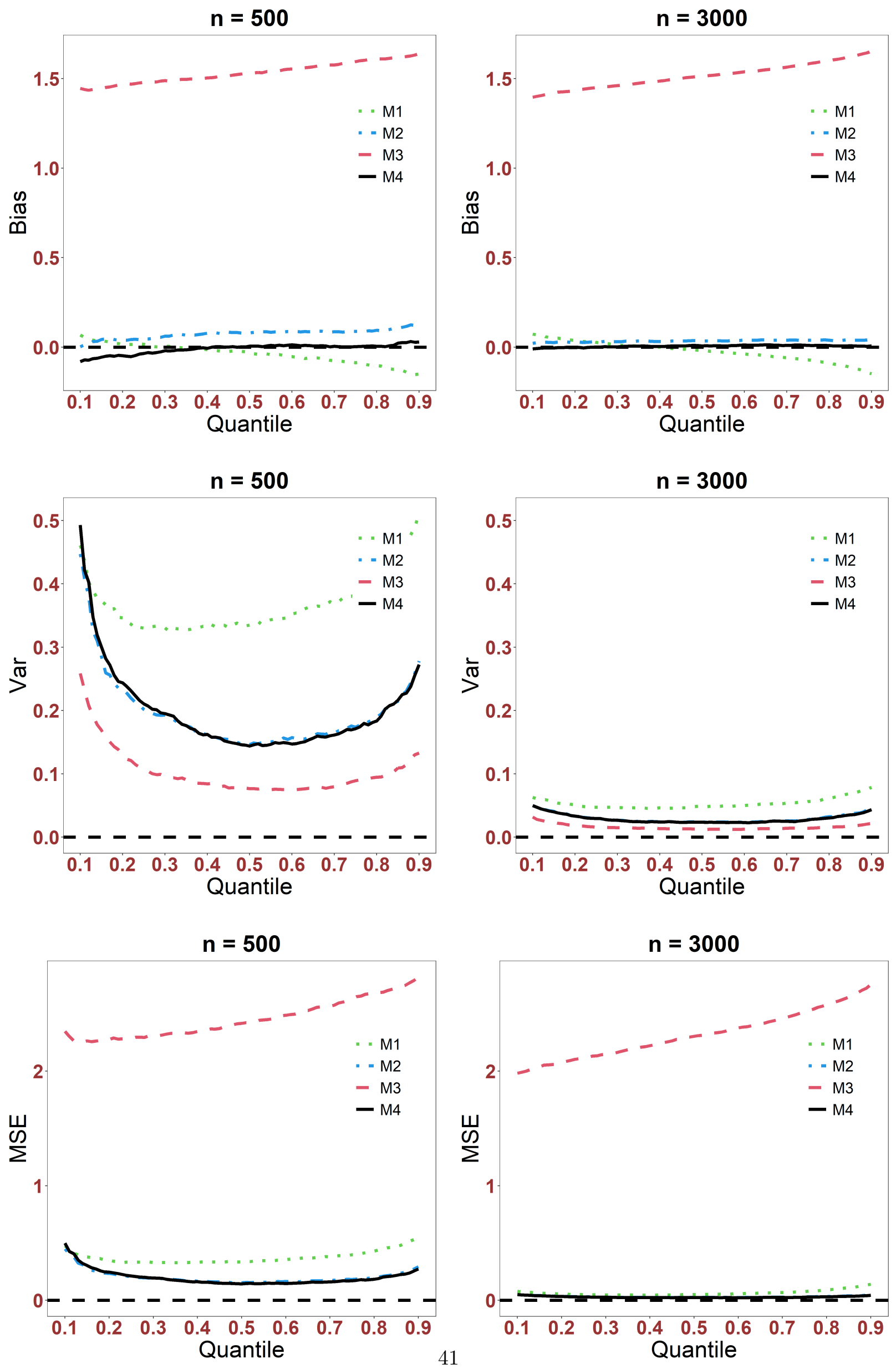

Figure 4: Bias, variance and MSE of the estimated conditional QTE for the compliers under different quantile levels when $\rho=0.5$. 

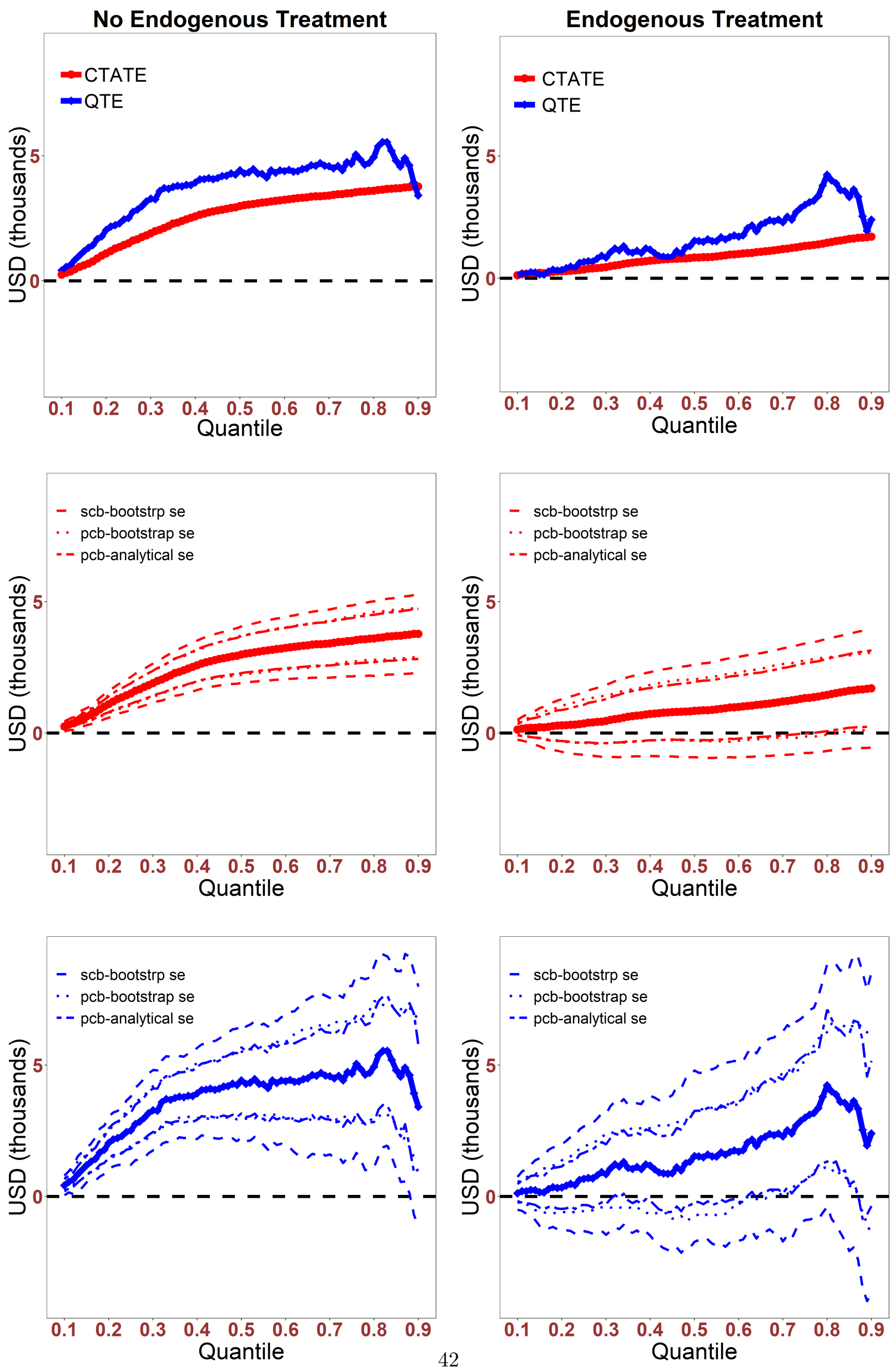

Figure 5: CTATE, QTE and the corresponding 95\% pointwise and simultaneous confidence bands: Adult men's earnings. 

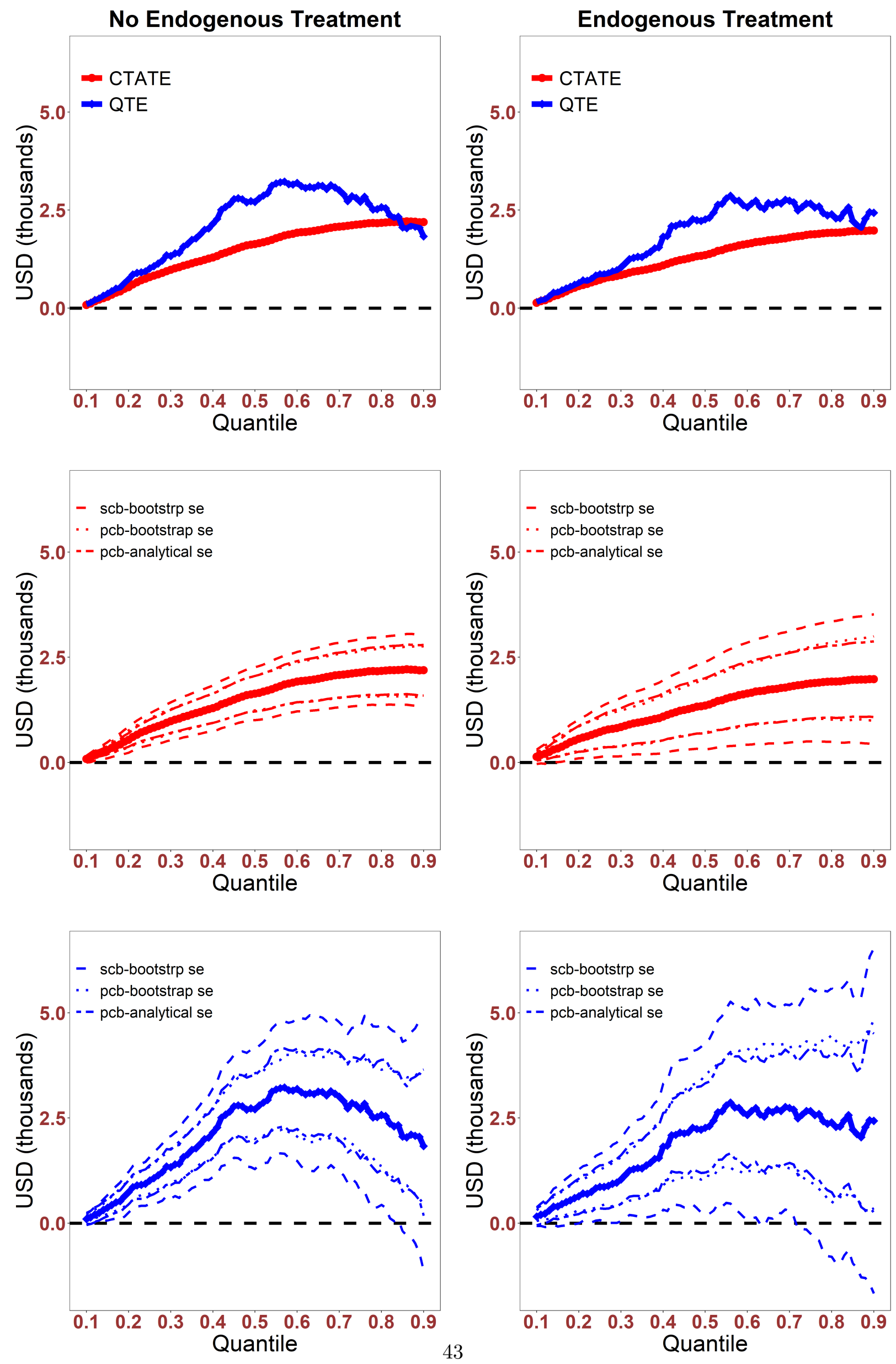

Figure 6: CTATE, QTE and the corresponding 95\% pointwise and simultaneous confidence bands: Adult women's earnings. 

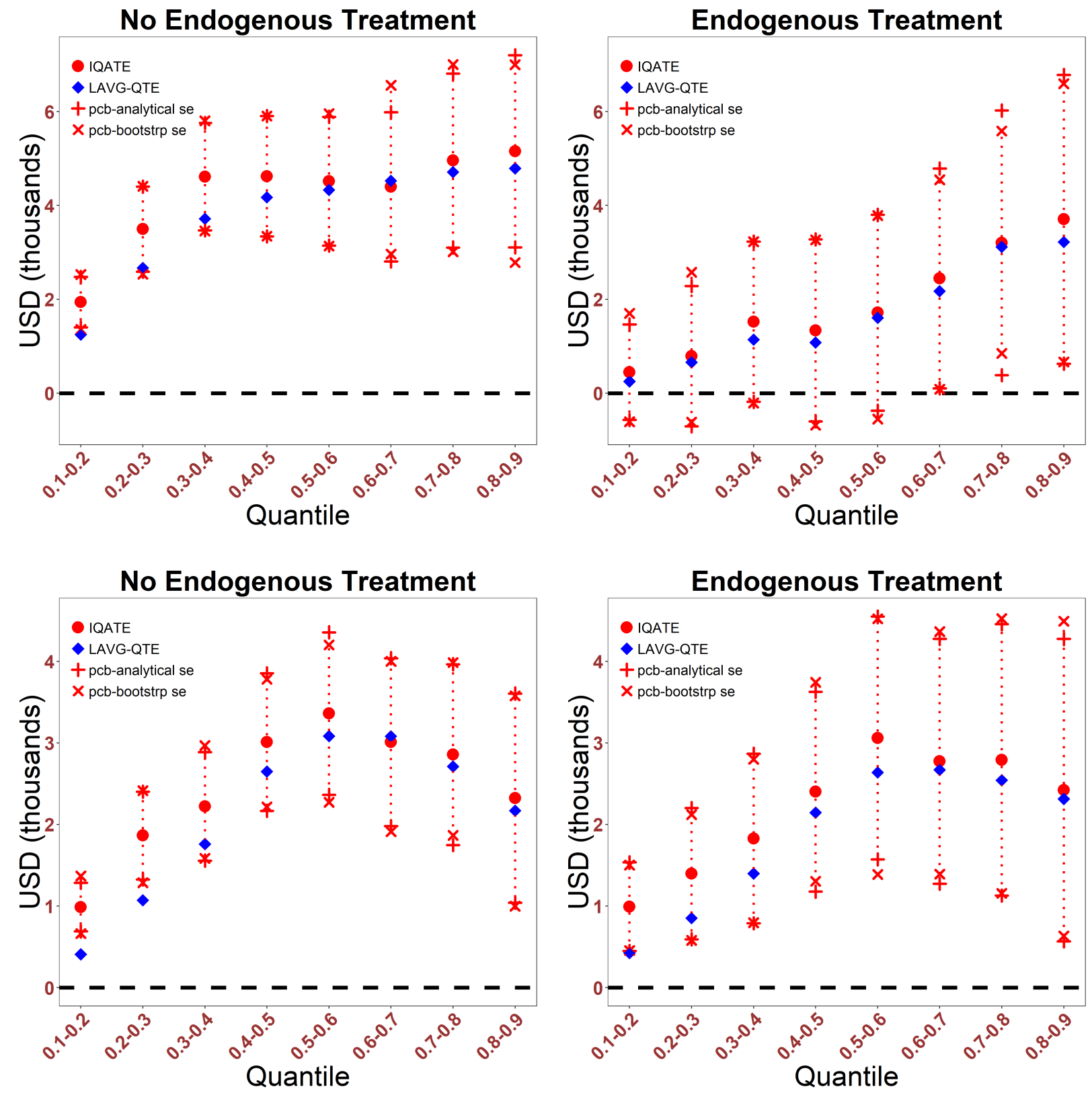

Figure 7: IQATE and 95\% pointwise confidence bands. Upper panel: Adult men's earnings. Lower panel: Adult women's earnings. 